\title{
Mitochondrial Dysfunction and Protein Aggregation in the Cybrid Cell Model of Parkinson's Disease
}

\author{
Emily Cronin-Furman \\ Chapel Hill, North Carolina
}

B.S., University of Miami (FL), 2007

\begin{abstract}
A Dissertation presented to the Graduate Faculty
of the University of Virginia in Candidacy for the Degree of

Doctor of Philosophy
\end{abstract}

Department of Neuroscience

University of Virginia

August, 2013 


\begin{abstract}
Parkins on's disease (PD) is the most common motor neurodegenerative disease that affects approximately 1 million patients in the United States. The cause of PD in a majority of patients is currently unknown. PD neuropathology is characterized by a loss of dopaminergic (DA) neurons and the formation of intracellular protein aggregates, called Lewy bodies (LB). The presence of these protein aggregates suggests that a decline in protein degradation may play an important role in PD pathogenesis. In addition, mitochondrial dysfunction has also been reported in PD patient brain and periphery. In an effort to better understand PD pathogenesis, we investigated the relationship between mitochondrial function and prote in aggregation in the cybrid model of PD, which forms intracellular aggregates similar to LB, called cybrid LB (CLB). The role of LB formation in PD is controversial. In an effort to determine if CLB are detrimental or cytoprotective to cybrid cells, we selected cells containing CLB and generated sub-cloned cell lines. We anticipated that there would be a consistent change across the sub-cloned lines; however, instead we found that the heterogene ity between the different original PD cybrid lines produced variable mitochondrial phe notypes in the sub-cloned lines. This suggests that protein aggregation does not have a consistent relationship with mitoc hondrial function. Next, we expressed a yeast electron transport chain gene, Ndi1, to bypass the dysfunctional complex I in a PD cybrid cell line. We were able to significantly improve mitochondrial function by expressing Ndi1; however, we did not prevent CLB formation. The findings from these first two studies led us to investigate if autophagy was involved in both mitochondrial function and CLB formation. By inhibiting autophagy, we observed a significant decline in mitochondrial function,
\end{abstract}


suggesting autophagy plays an important role in maintenance of mitochondrial function. We also found that autophagic proteins are incorporated into the CLB, implicating a role for autophagy in CLB formation and growth. These studies indicate that there is an essential role for autophagy in both the maintenance of mitochondrial function, as well as CLB formation. These findings have uniquely advanced our understanding of the relationship between these two important processes in PD pathogenesis. 


\section{Table of Contents}

Title page

Abstract

Table of Contents

Abbreviations

Acknowledgements

\section{Introduction}

Parkins on's disease

Mitochondria and Parkinson's disease

Protein degradation, Lewy bodies, and Parkinson's disease

II. Gene ral Methods

Cybrid cell line culture $\quad 32$

Immunocytochemistry 33

Immunoblotting 34

Quantitative real-time polymerase chain reaction $\quad 35$

Live cell staining and analys is $\quad 35$

Cellular respiration 36

Electron microscopy

Statistical Analysis 37

III. Mitochondrial quality, dynamics, and functional capacity in Parkinson's 38 disease cybrid cell lines se lected for Lewy body expression

Introduction

Methods

Generation of sub-cloned cybrid lines based on CLB expression $\quad 40$

Nucleoid imaging and quantification 42

Results $\quad 42$

CLB morphology and composition in $\mathrm{PD}_{\text {Orig }}$ and $\mathrm{PD}_{\mathrm{CLB}}$ cybrid cell lines 42

Mitochondrial morphology in $\mathrm{PD}_{\text {Orig }}$ and $\mathrm{PD}_{\mathrm{CLB}}$ cybrid cell lines 43

Mitochondrial oxygen consumption in PD $\mathrm{P}_{\text {Orig }}$ and $\mathrm{PD}_{\mathrm{CLB}}$ cybrid cell lines 46

Nucleoid density in $\mathrm{PD}_{\text {Orig }}$ and $\mathrm{PD}_{\mathrm{CLB}}$ cybrid cell lines 49

Gene expression le vels for mitochondrial ETC genes in PD Orig

and $\mathrm{PD}_{\mathrm{CLB}}$ cybrid cell lines

Biogenesis gene expression in $\mathrm{PD}_{\text {Orig }}$ and $\mathrm{PD}_{\mathrm{CLB}}$ cybrid cell lines 55

Discussion $\quad 58$

IV. Expression of the yeast NADH dehydrogenase improves mitochondrial 64 function in a cell model of Parkinson's dise ase Introduction $\quad 64$

$\begin{array}{ll}\text { Methods } & 67\end{array}$

Ndil transfection $\quad 67$

Results $\quad 68$

Ndil expression in SH-SY5Y cells and a PD cybrid cell line 68

Ndil expression improves mitochondrial oxygen consumption 68 
Effect of Ndi1 expression of mitochondrial morphology 72

$\begin{array}{ll}\text { Ndil expression increased mitochondrial ge ne expression } & 72\end{array}$

$\begin{array}{ll}\text { Ndil expression increased mitochondrial biogenesis signaling } & 73\end{array}$

Ndil expression increased ETC complex assembly $\quad 74$

Expression of Ndi1 decreased total cellular aggregated prote in content but 75 but did not alter CLB expression

$\begin{array}{ll}\text { Discussion } & 76\end{array}$

V. Autophagy is essential for the maintenance of mitochondrial function $\quad 85$ and contributes to CLB formation in PD cybrid cell lines Introduction $\quad 85$

Methods $\quad 89$

BAF treatment $\quad 89$

$\begin{array}{lr}\text { LC3-GFP expression } & 90\end{array}$

Mitochondrial morphology 90

Results $\quad 90$

Treatment with BAF results in accumulation of autophagos omes 90

Inhibition of autophagy decrease mitochondrial respiration and leads to 93

fragmented mitochondrial morphology

Inhibition of autophagy increases aggregated prote in content 97

Autophagy components are found in CLB 97

Autophagosomes associate with CLB independent of lysosomal fusion $\quad 100$

$\begin{array}{ll}\text { Discussion } & 102\end{array}$

VI. Conclusions and Future directions 109

VII. References 


\begin{tabular}{|c|c|}
\hline & List of Abbre viations \\
\hline ANOVA & analys is of variance \\
\hline aSYN & alpha-synuc lein \\
\hline BAF & Bafilomycin A1 \\
\hline CLB & cybrid Lewy body \\
\hline CMA & chaperone mediated autophagy \\
\hline DA & dopaminergic \\
\hline DMEM & Dulbecco's modified Eagle medium \\
\hline ECAR & extracellular acidification rate \\
\hline EM & electron microscopy \\
\hline ETC & electron transport chain \\
\hline FBS & fetal bovine serum \\
\hline FCCP & carbonyl cyanide 4-(trifluoromethoxy)phenylhydrazone \\
\hline gDNA & genomic DNA \\
\hline GFP & green fluorescent protein \\
\hline GI & gastrointestinal \\
\hline GM & growth media \\
\hline LAMP2A & lysosome-associated membrane prote in 2 \\
\hline LB & Lewy body \\
\hline LC3 & microtubule-associated prote in light chain 3 \\
\hline LN & Lewy neurite \\
\hline LRRK2 & lecuine rich repeat kinase 2 \\
\hline MOI & multiplicity of infection \\
\hline $\mathrm{MPP}+$ & 1-methyl-4-phenylpyridinium \\
\hline MPTP & 1-methyl-4-phenyl-1,2,3,6-tetrahydropyridine \\
\hline mtDNA & mitochondrial DNA \\
\hline MTOC & microtubule organizing center \\
\hline mTOR & mammalian target of rapamycin \\
\hline NADH & nicotinamide adenine dinucleotide \\
\hline Ndil & NADH dehydrogenase internal \\
\hline NRF1 & nuclear respiratory factor 1 \\
\hline OCR & oxygen consumption rate \\
\hline OXPHOS & oxidative phosphorylation \\
\hline p62 & p62/squestosome 1 \\
\hline $\mathrm{PD}$ & Parkins on's disease \\
\hline PGC- $1 \alpha$ & peroxisome proliferator-activated receptor gamma coactivator 1 -alpha \\
\hline PINK1 & phosphatase and tens in homolog-induced kinase 1 \\
\hline qRT-PCR & quantitative real-time polymerase chain reaction \\
\hline RBD & rapid eye movement sleep behavior disorder \\
\hline ROS & reactive oxygen species \\
\hline SIRT1 & silent mating type information regulation 2 homolog \\
\hline $\mathrm{SNpc}$ & substantia nigra pars compacta \\
\hline SOD & superoxide dis mutase \\
\hline TFAM & mitochondrial transcription factor A \\
\hline TFBM1 & mitochondrial transcription factor B1 \\
\hline UCH-LI & ubiquitin C-terminal hydrolase L1 \\
\hline
\end{tabular}


UPS ubiquitin proteasome system 


\section{Acknowledgements}

This work would not have been possible without the support and assistance of several other people who deserve recognition. Kate Borland carried out the cloning procedure described in Chapter 3 and created Figure 3-1. Jennifer Barber-Singh of the Yagi lab at Scripps performed the Ndil transfections described in Chapter 4. Kristen Bergquist and Lauren Wright provided essential assistance with the tissue culture required for Chapters 3, 4, and 5. The qRT-PCR experiments described in Chapters 2 and 3 were performed with assistance from Dr. James Bennett.

I would like to thank Tracy Mourton, Nadia Badr Cempré, and the Neuroscience Graduate Program for the ir support and assistance. The input and advice from my committee members Drs. Jonathan Kipnis, Kevin Lee, James Mandell, and Scott Zeitlin was essential for the creation of this document. Finally, I would like to thank my mentor, Dr. Patricia Trimmer, for her support and endless patience throughout my graduate career. This dissertation would not have been possible without her invaluable input and guidance.

This work was supported by the Neurosc ience Training Grant (5T32GM00832817), the Morris K. Udall Parkinson's Research Center of Excellence grant (7P50 NS039788-11), and the Medical College of Virginia Foundation. This work would not have been possible without the continued support of the Movers \& Shakers and other generous community members whose philanthropy enabled this project, as well as the Parkins on's and Movement D isorders Center staff at Virginia Commonwealth University. 


\section{Introduction}

Parkins on's disease (PD) is a neurodegenerative disease that is neuropathologically characterized by the loss of dopaminergic (DA) neurons and the formation of intracellular, prote inaceous aggregates called Lewy bodies (LB). Although familial forms of PD exist, most cases are sporadic, without a known etiology. In light of the different familial forms of PD, two processes have emerged as being critically involved in PD pathogenesis: the loss of mitochondrial respiratory function and the accumulation of aggre gated proteins. Several of the genes found to cause familial PD relate to mitochondrial quality control and/or protein degradation. In addition to familial $\mathrm{PD}$, sporadic patients also have prote in aggregation resulting in LB formation and reduced mitochondrial function, which further implicates a role for these two processes in PD pathogenesis. A loss of mitochondrial respiratory function, leading to a decline in ATP production, can have dramatic consequences for cell survival. Likewise, the failure of protein proteolysis pathways to degrade dysfunctional orga nelles or misfolded proteins can lead to the formation of intracellular proteinaceous inclusions, such as LB. A better understanding of these pathways in the context of PD is essential to our understanding of PD pathogenesis and vital for the creation of better therapeutic approaches. The next sections will describe the symptoms and pathology of PD and the consequences of mitochondrial dysfunction, as well as explore how LB formation could be a result of a failure of the protein degradation pathways.

\section{Parkins on's dise ase}

Parkinson's disease symptoms and pathology 
$\mathrm{PD}$ is the most common motor neurodegenerative disorder. PD is commonly diagnosed due to one or more of the four cardinal motor symptoms, tremor, bradykinesia, rigidity, and postural instability, accompanie d by a clinical response to dopamine replacement therapy (Hughes et al., 1992; Savitt et al., 2006). These motor symptoms have been linked to a specific vulnerability of DA neurons in the midbrain that function as part of the basal ganglia circuitry. In addition to these motor symptoms, several nonmotor symptoms are also pre valent in PD patients, including olfactory, circadian, autonomic, and gastrointesintestinal dysfunction, some of which develop before motor symptoms (Chaudhuri and Schapira, 2009; Postuma et al., 2013; Ross et al., 2008). For example, rapid eye movement sleep behavior disorder (RBD) is considered a prodromal symptom for PD. Studies of patients with RBD who have gone on to develop PD or another synucleinopathy have autonomic dysfunction as early as 20 years before the appearance of motor symptoms (Postuma et al., 2013). Gastrointestinal (GI) symptoms of constipation and reduced bowel movement have also been suggested as early symptoms of PD (Abbott et al., 2001). A recent report suggests that low GI tract motility may also be associated with a decrease in nigral neuron density (Petrovitch et al., 2009). In addition to these symptoms, many patients can also develop cognitive symptoms, including mild cognitive impairment and dementia (Svenningsson et al., 2012). Many of these non-motor symptoms can be linked to a loss of DA neurons in extranigral areas, such as the olfactory bulb, autonomic nervous system, and enteric nervous system.

The motor symptoms are normally treated by dopamine replacement therapy (LDOPA); however, this approach does not necessarily improve the non-motor symptoms of PD nor does it alter disease progression. L-DOPA has been the gold standard in PD 
treatment for the past 30 years; however many new studies are underway to test new therapeutic approaches to treating and slowing the progression of $\mathrm{PD}$, such as the one described in Chapter 4. There has also been a strong focus on developing treatments for the non-motor symptoms of PD, especially mild cognitive impairment and dementia, as these symptoms can have significant effects on patient quality of life (Svenningsson et al., 2012). The exact cause of PD is unknown and a better understanding of PD pathogenes is is vital for the development of better therapeutics to treat this disease. Currently, there is no cure for PD.

\section{PD pathogenesis}

Approximately 90\% of PD cases are considered sporadic, with no known etiology; however, several risk factors have been implicated, including aging, pesticide exposure, exposure to heavy metals, certain genetic mutations, and head injuries, such as concussions. Development of PD may be a result of a combination of these or other unknown factors. The most prevalent risk factor is aging, as 60 is the average age of onset (Olanow et al. 2009). Several groups have proposed that PD develops due to multiple hits, such as aging and inflammation or oxidative damage and protein aggregation (Tsang and Chung, 2009). Many of these risk factors interact on multiple levels, in particular the relationship between mitochondrial function and prote in aggregation, which will be discussed in later sections. Progression and severity of symptoms can vary greatly, leading to an extremely heterogeneous population (Olanow et al., 2009). Non-sporadic cases of PD, due to either familial mutations or known neurotoxin exposure, have aided in the understanding of PD pathogenesis and have led to the creation of several important models. 
Mitochondrial neurotoxin expos ure has been described as a potential cause of parkinsonism. In two separate cases of exposure to 1-methyl-4-phenyl-1,2,3,6tetrahydropyridine (MPTP), patients self-administered impure designer drugs and developed sudden onset of classic parkinsonian symptoms, such as bradykinesia, tre mor, and a shuffling gait (Langston et al., 1983). Post-mortem analys is of some of these patients showed neuronal cell loss in the substantia nigra, suggesting that this compound is specifically toxic to the midbra in neurons (Langston et al., 1999). Astrocytes take up MPTP and release the metabolite 1-methyl-4-phenylpyridinium (MPP+) that is then taken up by the dopamine transporter into dopaminergic neurons (Javitch et al., 1985). Intracellular MPP+ is selectively toxic to complex I of the mitochondrial electron transport chain (Mizuno et al., 1987). This can lead to a decline in ATP production as well as increased levels of free radical formation and oxidative stress.

Pesticides, such as rotenone and paraquat, have also been implicated in the development of parkinsonism. Rotenone binds with high affinity to complex I, similar to MPP+ (Betarbet et al., 2000). In vitro, rotenone can reduce spare respiratory capacity and increase free radical formation (Yadava and Nicholls, 2007). Although rotenone toxicity is a useful model for PD in cell culture and animals, the casual link between rotenone exposure and development of PD remains unclear in humans. One study reported that exposure to pesticides alone may not lead to PD; however, coupled with certain rare genes, there may be an increased genetic susceptibility (Elbaz and Tranchant, 2007). Conversely, a more recent study showed that there is a positive association between exposure to rotenone or paraquat with the development of PD (Tanner et al., 
2011). Many of these epidemiological studies on the relationship between environmental exposures and PD are still underway.

Approximately $10 \%$ of PD patients have a familial mutation that has been linked to the development of PD. The manifestations of the disease vary between the different gene mutations. There are differences in age of onset, presence or absence of associated dementia, response to L-dopa therapy, and presence or absence of LB (Mizuno et al., 2008). Several of the different mutations have aided in our understanding of PD pathogenes is due to the ir relevance to protein degradation, mitochondrial dysfunction, or both.

One of the first to be described was the gene for aSYN, SNCA, which causes autosomal dominant familial PD (Polymeropoulos et al., 1997). This mutation can exist as a missense mutation (A53T, A30P, or E46K) or in duplicate or triplicate (ChartierHarlin et al., 2004; Polymeropoulos et al., 1997; Singleton et al., 2003). Importantly, aSYN has also been identified as a major component of Lewy bodies (LB) (Spillantini et al., 1997), which will be discussed in a later section. The normal functions of aSYN are not completely understood, although there is evidence that aSYN can associate with membranes and play a role in synaptic vesicle release (Tofaris and Spillantini, 2007). Knockout mice lacking the $S N C A$ gene are viable but show alterations in DA release, which supports a proposed role of aSYN in vesic le docking (Abeliovich et al., 2000a). Mutations in the SNCA gene can increase the propensity of the protein to aggregate, possibly due to changes in the secondary structure of the protein (Polymeropoulos et al., 1997). Although these mutations can explain the appearance of aSYN aggregates in 
patients with one of these mutations, it does not explain the aggregation of wild-type aSYN seen in sporadic PD patients.

Several genes have also been identified that have a direct relationship with mitochondrial quality control. Parkin is an E3 ligase involved in the poly-ubiquitination of proteins targeted for degradation by the proteasome (Zhang et al., 2000) and causes an early onset autosomal recessive form of familial PD (Kitada et al., 1998). Recent studies have shown that parkin has functions that are independent of the of E3 ligase activity, such as a role in mitochondria biogenesis (Shin et al., 2011). PINK1 (phos phatase and tensin homolog-induced kinase 1) is a mitochondrial-associated protein involved in the cellular stress response that is also associated with the early onset autosomal recessive form of PD (Valente et al., 2004a; Valente et al., 2004b). Mutations in the PINK1 gene are in the kinase domain, resulting in a loss-of-function of the PINK1 protein (Valente et al., 2004a). Both parkin and PINK1 have been shown to play a role in the turnover of dysfunctional mitochondria via autophagy, which will be discussed in a later section. Mutations have als o been described in two other proteins related to mitochondria or prote in degradation. The first is the mitochondria-associated protein DJ-1, a prote in involved in the cellular response to oxidative stress (Bonifati et al., 2003). The second is ubiquitin C-terminal hydrolase L1 (UCH-L1), a hydrolase enzyme involved in the ubiquitin-proteasomal system (Leroy et al., 1998). Similar to parkin and PINK1, UCHL1 is involved in prote in degradation. These monogenic forms of PD strengthen the hypothesis that PD pathogenesis is related to both mitochondrial function and cellular degradation processes (Dauer and Przedborski, 2003). 
Mutations in the leucine-rich repeat kinase 2 (LRRK2, dardarin) are associated with late onset PD, which may have been previously described as idiopathic (Paisan-Ruiz et al., 2004; Zimprich et al., 2004). LRRK2 is large protein, approximately $250 \mathrm{kDa}$, found in the brain and several other organs in the body (Giasson et al., 2006). The function of this gene is largely unknown, but it has both kinase and GTPase activities (Rudenko et al., 2012). This is the most common gene implicated in familial PD (Kle in and Schlossmacher, 2007); however, it is currently unknown how the various mutations in this gene cause the disease. There is evidence that mutations in LRRK2 can increase mitochondrial de gradation, potentially by altering calcium homeostasis, and lead to neurite shortening (Cherra et al., 2013). This suggests that, similar to other familial forms of PD, LRRK2 mutations may effect mitochondrial function, as well as protein degradation.

Unlike the other monogenic mutations, the penetrance of LRRK2 is variable between different ethnicities and specific mutations. In fact, some of the LRRK2 mutations may not directly cause PD, but instead represent a risk factor for the disease (Lesage and Brice, 2009). The study of the effects of LRRK2 mutations is complicated by the high number of different mutations, which has made elucidating the exact pathogenic mechanism caused by mutations in this protein more difficult (Cookson, 2012). Further characterization of the functional consequences of LRRK 2 mutations will help to elucidate the mechanism by which these mutations in this protein can lead to PD.

The differences between patients with LRRK2 mutations are an example of the heterogene ity that is commonly observed in the PD patient population. The discovery of these different genes that can cause familial PD have certainly advanced our 
understanding of the disease; however, the models created using these different mutations have not resulted in the development of better therapeutics for PD (Muller, 2010; Waldmeier et al., 2006). This is a prime example of how studying a heterogeneous disease can be difficult in animal mode ls that study the mutations found in less than $10 \%$ of patients. Instead of using models based on familial PD, we believe that the models developed using material from sporadic PD patients, such as platelets, will help us gain a better understanding of the etiology of PD.

General pathology

PD is neuropathologically defined by the loss of neuromelanin containing dopaminergic neurons of the substantia nigra pars compacta (SNpc) (Figure 1-1) and the presence of LB in remaining dopaminergic neurons (Forno, 1996; Hirsch et al., 1988). The structure of LB will be described in a later section. The SNpc is not the only location of pathology in PD. Neuron loss and LB are

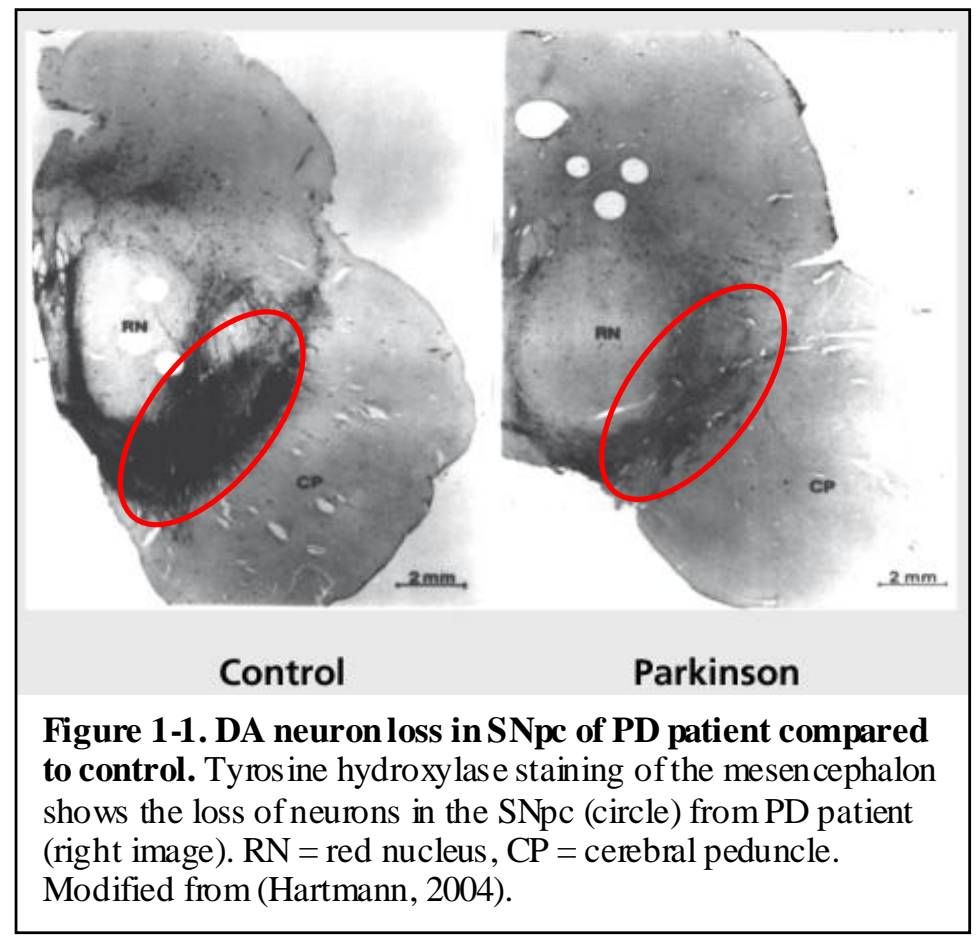
found in several extranigral areas, including the cortex, locus ceruleus, dorsal motor nucleus of the vagus, and the olfactory bulbs (Braak et al., 2001; Braak et al., 2006; Braak et al., 2003; Del Tredici et al., 2002; Forno, 1996). Pathology is also present 
outside of the brain in some autonomic nervous system ganglia, the GI system, and the spinal cord (Del Tredici and Braak, 2012; Den Hartog Jager and Bethlem, 1960). The presence of disease pathology outside of the SNpc may be related to the non-motor symptoms found in PD patients.

An extensive investigation of extranigral pathology in post-mortem PD patient tissue by Braak et al. has resulted in a proposed neuropathological staging of PD. The appearance of this pathology in the different nuclei established a model of progressive insult, stemming from the brainstem nuclei that innervate the enteric nervous system, progressing to the SNpc, and eventually including the cortex (Braak et al., 2003; Del Tredici et al. 2002). This model is supported by the finding that GI and autonomic dysfunction may precede motor symptoms in some patients (Abbott et al., 2001).

Although the Braak staging system is the result of extensive pathological evaluation of post-mortem PD patients, it is not without controversy. The major criticism of this model is that pathology does not always correlate precise ly with clinical disease behavior nor the sequence of symptoms in some patients (Halliday et al., 2012). Recently, support for the Braak staging system has come from studies of cell-to-cell transmission of aSYN aggregates, suggesting that aggregated aSYN may exhibit a prionlike behavior that results in the spread of neuropathology observed by Braak et al. (Hansen and Li, 2012). At this point, it is unclear if cell-to-cell aSYN transmission is the mechanism behind the progression of PD pathology, although it certainly warrants further study.

As the mechanis m behind the spread of PD pathology remains a mystery, understanding more about the nature of LB, in particular how they are formed, may aid in 
the explanation of PD pathogenesis. Importantly, LB represent a unifying pathological feature between the motor and non-motor symptoms, as LB are present in motor, as well as non-motor nervous system areas (Braak et al., 2006; Del Tredici and Braak, 2012; Del Tredici et al., 2002). Although the presence of LB is used as a neuropathological diagnostic hallmark, it is not the only pathology observed in PD patients. It is important to investigate the interactions between the formation of protein aggregates and the other pathological features of $\mathrm{PD}$, such as mitochondrial dysfunction. The next sections will describe the mitochondrial dysfunction observed in PD, as well as LB and the related proteolytic processes that could be involved in LB formation.

\section{Mitochondria and Parkins on's dise ase}

Mitochondrial structure and function

Studies on the origins of eukaryotic cells suggest that mitochondria themselves may be descendants of an aerobic $\alpha$-proteobacterium-like ancestor (Dyall et al., 2004). One theory is that the ancestors of modern day eukaryotes engulfed these mitochondriaancestors. The relationship that evolved between these two cell types is considered to be an endosymbiotic relationship. It is possible that the mitochondrial-like proteobacteria detoxified the oxygen rich environment as well as provided energy to the host, while the host cells protected the bacteria from the environment (Dyall et al., 2004).

Support for this theory comes from the unique structure of the mitochondrial genome. Modern day mitochondrial DNA (mtDNA) exists as a circular molecule, similar to the bacterial ge nome (Gray et al., 1999) (Figure 1-2B). The mitochondrial genome is packaged into nucleoids and, unlike nuclear DNA, is not histone coated (Figure 1-2C). 
The present day human

mtDNA encodes only

thirteen prote ins for the

mitochondrial electron

transport chain (ETC), as

well as transport RNA

and ribosomal RNA

responsible for the

translation of these genes

(Anderson et al., 1981).

All of the other genes

responsible for

mitochondrial structure

and function are nuclear

encoded. Over the course

of evolution, there have

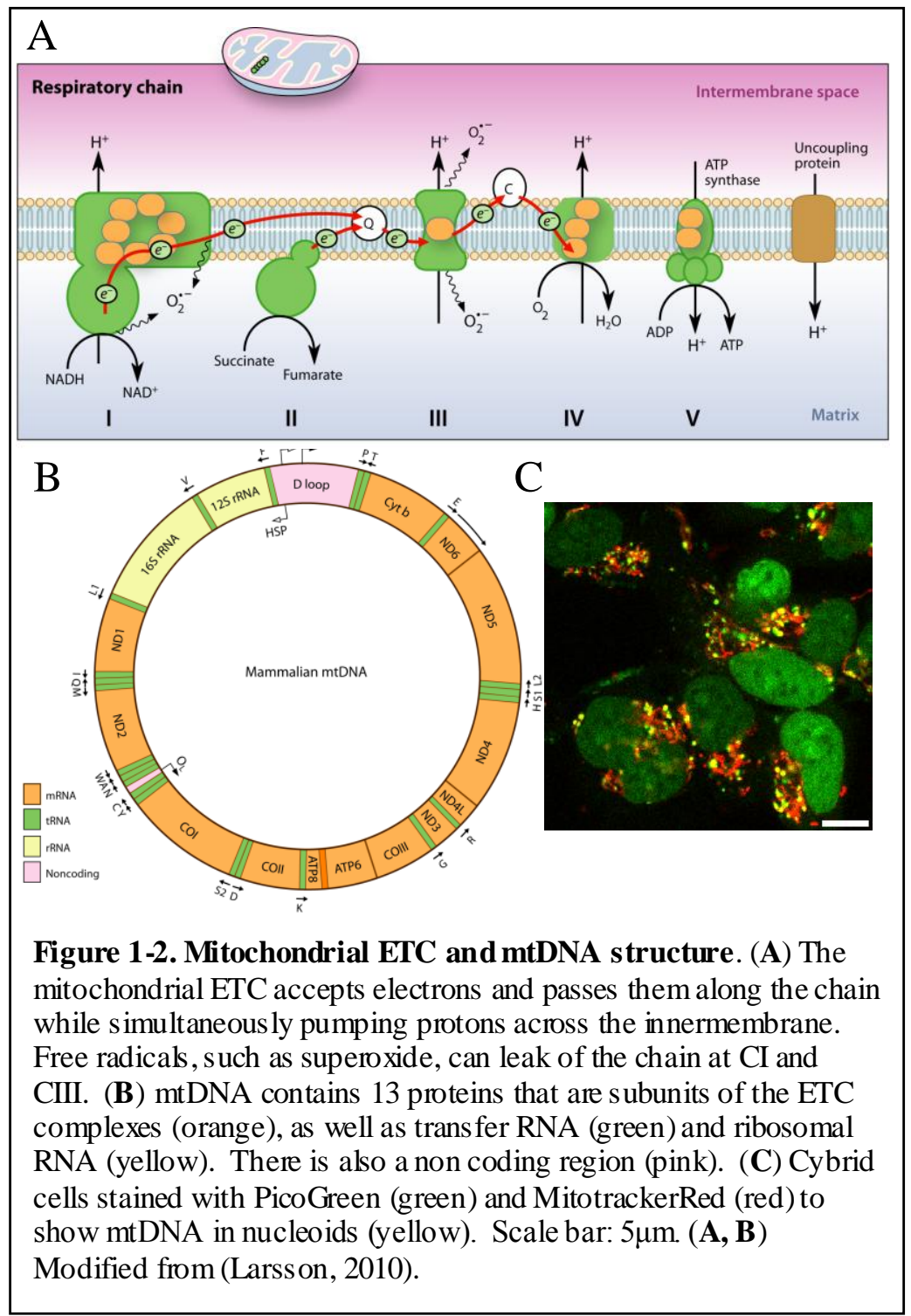

been considerable changes made to the mitochondrial genome, most notably a reduction in size, likely due to the transfer of much of the original genetic material from the mitochondria to the nucleus (Gray et al., 1999). The transfer of this genetic material from the mitochondrion to the nucle us is an interesting phenomenon, which is not entirely understood (Dyall et al., 2004).

In response to this exchange of genetic material and downsizing of the mitochondrial ge nome, mitochondria also evolved a complex import system to allow 
nuclear-encoded prote ins to be synthesized in the cytoplasm, inserted into the mitochondria, and localized to the one of the four mitochondrial compartments (Gray et al., 1999). Present day mitochondria are double membrane organelles, with an outer membrane and an inner membrane, both rich in phospholipids. In between these two membranes is a space known as the intermembrane space. The inner membrane is folded to create invaginations, called cristae, and surrounds the mitochondrial matrix. The mtDNA is found within the matrix, which is also where mitochondrial gene expression takes place using the mitochondrial ribosomes (reviewed in As in-Cayuela and Gustafsson, 2007). Importantly, this places mtDNA in close proximity to the ETC prote ins that span the inner membrane. Electrons can leak off the ETC at different sites, most commonly complexes I and III, to form free radicals in the mitochondrial matrix (Fiskum et al., 2003). The proximity of free radical production to mtDNA makes the mitochondrial ge nome susceptible to damage from free radicals produced locally by the ETC.

Mitochondria are the so-called powerhouse of the cell. Although the metabolism of glucose via glycolys is can produce some ATP, the mitochondrial ETC is responsible for the $80 \%$ of cellular ATP production by oxidative phosphorylation (OXHPOS) (reviewed in Papa et al., 2012) (Figure 1-2A). Mitochondria are also involved in the maintenance of cellular homeostas is, by contributing to calcium buffering and free radical signaling. Mitochondria play an important role in apoptosis, which strengthens their essential role in cell survival (Galluzzi et al., 2012).

The process of ATP production by the ETC, called OXPHOS, was first described in 1961 (Mitchell, 1961). During OXPHOS, NADH (nicotinamide adenine dinucleotide) 
from the Krebs cycle is oxidized by complex I (CI, NADH dehydrogenase) (reviewed in Papa et al., 2012). The iron-sulfur centers of CI accept the resulting electron and pass it on to coenzyme Q. The acceptance and passage of electrons occurs at the same time as hydrogen ions are pumped across the inner membrane into the intermembrane space. The electrons are passed to complex III (CIII, cytochrome $b c_{1}$ complex) and carried to complex IV (CIV, cytochrome oxidase) by cytochrome c. CIII can also accept electrons from complex II (CII, succinate dehydrogenase)via coenzyme Q. At CIV, electrons and oxygen are combined with hydrogen to make water, one of the byproducts of oxidative phosphorylation. Additional hydrogen ions are also pumped across the inner membrane by CIII and CIV, leading to the formation of an electrochemical gradient across the inner membrane. Complex V (CV, ATP synthase) harnesses the energy of this gradient to combine inorganic phos phate with ADP to make ATP. This process is also known as OXPHOS due to the consumption of oxygen as the electron acceptor and the phosphorylation of ADP.

The morphology of the inner membrane of mitochondria is intrinsically linked to the function of the ETC (Zick et al., 2009). The separation of the matrix and the intermembrane space by the inner membrane is required for the formation of the electrochemical gradient, which is generated when hydrogen ions are pumped across the inner membrane. This gradient als o establishes the mitochondrial membrane potential, which serves as a regulator of several mitochondrial functions, including mitochondrial fission. Alterations in the mitochondrial cristae, matrix, or membrane potential may have functional consequences, such as an increase in free radicals and a decrease in ATP production (Campello and Scorrano, 2010) 
Mitochondria produce free radicals as a natural byproduct of OXPHOS.

Although free radical signaling is part of normal cell function, mitochondria are equipped with several enzymes to deal with excess free radical production, such as superoxide dismutase (SOD) (Weisiger and Fridovich, 1973). The SOD family of enzymes catalyzes the conversion of superoxide to hydrogen peroxide. Hydrogen peroxide can subsequently be made into water via catalase, glutathione peroxidase, and peroxiredoxin III. These enzymes, along with many others, are essential for handling of excess free radicals that are produced during normal and abnormal mitochondrial function.

Part of the complexity of the ETC is due to the genetic origins of individual complex subunits. Four of the complexes of the ETC are made up of proteins encoded by both the nuclear and mitochondrial genomes. Mitochondria can also contain multiple copies (between 1-15) of the mtDNA genome (Satoh and Kuroiwa, 1991). Due to the relative ly high mutation rate of mtDNA mutations relative to genomic DNA (gDNA), there can be variability among the mtDNA copies contained within each cell or even within an individual mitochondrion. This can result in different combinations of wildtype and mutated mtDNA in different tissues, cells, and even individual mitochondria. This heterogeneity of mitochondrial ge notypes is called heteroplasmy (Linnane et al., 1989).

Coordination of mtDNA and gDNA gene expression is essential for proper maintenance of mitochondrial function, especially in cells like neurons where mitochondria may be found at the terminal ends of distal axons. Several, if not all, nuclear-encoded ETC subunits are transcribed and synthesized as precursor proteins (Liu and Wong-Riley, 1994). These nuclear-encoded subunits are expressed in excess of the 
cell's need and stored in the mitochondrial matrix as pro-form (Hundt et al., 1980). As new ETC complexes are assembled, these proteins are cleaved and inserted into the complexes (Liu and Wong-Riley, 1994).

The transcription factors involved in both mtDNA transcription and gDNA transcription are both regulated upstream by PGC- $1 \alpha$ (Peroxisome proliferator-activated receptor gamma coactivator 1-alpha) (Scarpulla, 2008). PGC-1 $\alpha$ is considered to be the master regulator of mitochondrial biogenesis (Wu et al., 1999). PGC-1 $\alpha$ is activated by a host of different signaling pathways including free radicals, sirtuins, cyclic AMP, and exercise (Wenz, 2011). PGC-1 $\alpha$ controls the activity of several DNA-binding proteins and transcription factors that control both mitochondrial biogenesis, mtDNA gene expression, and nuclear-encoded mitochondrial gene expression, such as nuclear respiratory factor 1 (NRF1), mitochondrial transcription factor B1 (TFB1M), and mitochondrial transcription factor A (TFAM) (reviewed in Hock and Kralli, 2009). Mitochondrial biogenes is is an important part of the maintenance of mitochondrial function. As the next sections will show, compromised mitochondrial function has been implicated to play a central role in PD pathogenesis.

\section{Mitochondrial dysfunction in Parkinson's disease}

There are several lines of evidence implicating a central role for mitochondria in PD pathogenesis. As described above, both MPTP and rotenone have been found to cause parkinsonism, suggesting a link between mitochondrial dysfunction and PD. Both of these compounds are specific CI inhibitors. In light of these findings, several groups measured ETC enzymatic activity in the brain and periphery of PD patients. Reduced CI activity has been observed in PD patients tissues (Parker et al., 1989; Schapira et al., 
1990a; Schapira et al., 1990b). A large genome-wide analysis of neuronal and blood cells of PD patients found that expression of mitochondrial genes, specifically those regulated by PGC-1 $\alpha$, was reduced (Zheng et al., 2010). Taken together, these studies show that mitochondrial dysfunction is an important part of PD pathogenesis.

Free radicals, such as reactive oxygen species (ROS) and reactive nitrogen species, can cause oxidative damage to proteins, lipids, and nucleic acids in mitochondria. In particular, ROS can cause strand breakages and base modifications (Floyd and Carney, 1992). Oxidative damage of mtDNA could also lead to an increase in mutations, such as deletions and base pair substitutions during DNA replication (reviewed in Lagouge and Larsson, 2013). There are mtDNA deletions found in the nigra of both PD patients and the general aging population (Bender et al., 2006). Specifically, there are deletions in the region of the genome that encodes subunits of CIV that can lead to reduced ETC activity. In addition to the observed defic its in CI of PD patients, decreases in CIV function is found in the SNpc more often than other areas of the brain (Kraytsberg et al., 2006). The accumulation of deletions in mtDNA can affect the function of CI in particular, because CI has seven mtDNA-encoded subunits, which is more than any of the other ETC complexes. Based on these findings, we can speculate on two of the possible explanations. First, the spontaneously occurring mutations, which the SNpc accumulates more quickly than other regions of the brain, are causing mitochondrial dysfunction, free radical production, and oxidative stress. The second possibility is that mitochondrial dysfunction occurs without mutation, resulting in oxidative stress and free radical production, which then causes mtDNA mutations. It has been shown that dopaminergic neurons are highly vulnerable to oxidative stress due to 
metabolites of dopamine oxidation (Olanow et al., 2009). In fact, the SNpc is a highly oxidative environment (Dexter et al., 1989), so it is feasible that increases in oxidative damage to mitochondria over time could lead to neuron loss. There is also evidence of oxidatively damaged aSYN in the SNpc, which could contribute to LB formation (Giasson et al., 2000). Increased lipid peroxidation and oxidatively dama ged mitochondrial DNA have also been observed in the SNpc of PD patients (Arthur et al., 2009; Yoritaka et al., 1996). Furthermore, there is also evidence of reduced antioxidant activity in SNpc of PD patients (Kish et al., 1985).

Studies described above have shown mtDNA deletions and reduced mitochondrial function are both present in the brain of PD patients. However, it is currently unknow $n$ how these mitochondrial deficits play a role in PD pathogenesis. For our studies, we investigated whether mtDNA from patients was sufficient to recapitulate the pathological hallmarks of PD, which would suggest that mitochondrial function has an essential role to play in PD pathogenesis. Our model, described in a later section, has given us important insights into the cellular consequences of mitochondrial dysfunction. This model has also allowed us to address the relationship between mitochondrial dysfunction and one of the other neuropathological hallmarks of PD, LB formation. The next sections will address the involvement of prote in proteolytic pathways in both prote in aggregation and maintenance of mitochondrial function. 


\section{Protein degradation, Lewy bodies, and Parkinson's dise ase}

Protein degradation pathways

Protein turnover is an important part of normal cellular function. Proteins can become misfolded or damaged and require cellular proteolytic systems to be degraded back into basic biomolecules, which can then be reused to build new proteins. The ubiquitin proteasomal system (UPS) is one of the primary methods of protein degradation; however, the next sections will focus primarily on the process macroautophagy that degrades aggregated proteins, such as aSYN, and dysfunctional organe lles, such as mitochondria.

Macroautophagy, referred to from now on as autophagy, is responsible for degradation of many cytoplas mic elements including organelles. Autophagy is an important cellular process for catabolic metabolism, whereby cells breakdown cytosolic components, such as proteins and organelles, for reuse by the cell instead of metabolizing exogenous nutrients. Autophagy can be initiated by stress, starvation, or artificial conditions; however, in many cells there is constitutive activation of autophagy (Bhaskar and Hay, 2007; van Sluijters et al., 2000). Post-mitotic cells, such as neurons, rely on high levels of autophagy to maintain normal cellular function (Xilouri and Stefanis, 2011). Chaperone-mediated autophagy (CMA) is a related process, wherein chaperones mediate the insertion of proteins directly into lysosomes through receptors in the lysosomal membrane (Kaushik and Cuervo, 2012)

The ma in activator of autophagy is the large-multi-domain protein called mammalian target of rapamycin (mTOR) (Noda and Ohsumi, 1998). This protein simultaneously regulates autophagy and prote in translation. When active, mTOR induces 
prote in trans lation while inhibiting autophagy. However, when mTOR is inhibited, it no longer inhibits Atg 13 (Autophagy protein 13) through phosphorylation (Abeliovich et al., 2000b). This release of mTOR inhibition on Atg13 is considered an initiating step in autophagy (Jung et al., 2009). After autophagy initiation, several more autophagy-related (Atg) proteins are activated, ultimately leading to autophagos ome formation and engulfment of cargo (Figure 1-3) (Yang and Klionsky, 2009). There are also pathways of initiating autophagy that are mTOR independent, called non-canonical pathways; however, this is outside the scope of this dissertation (Codogno et al., 2012).

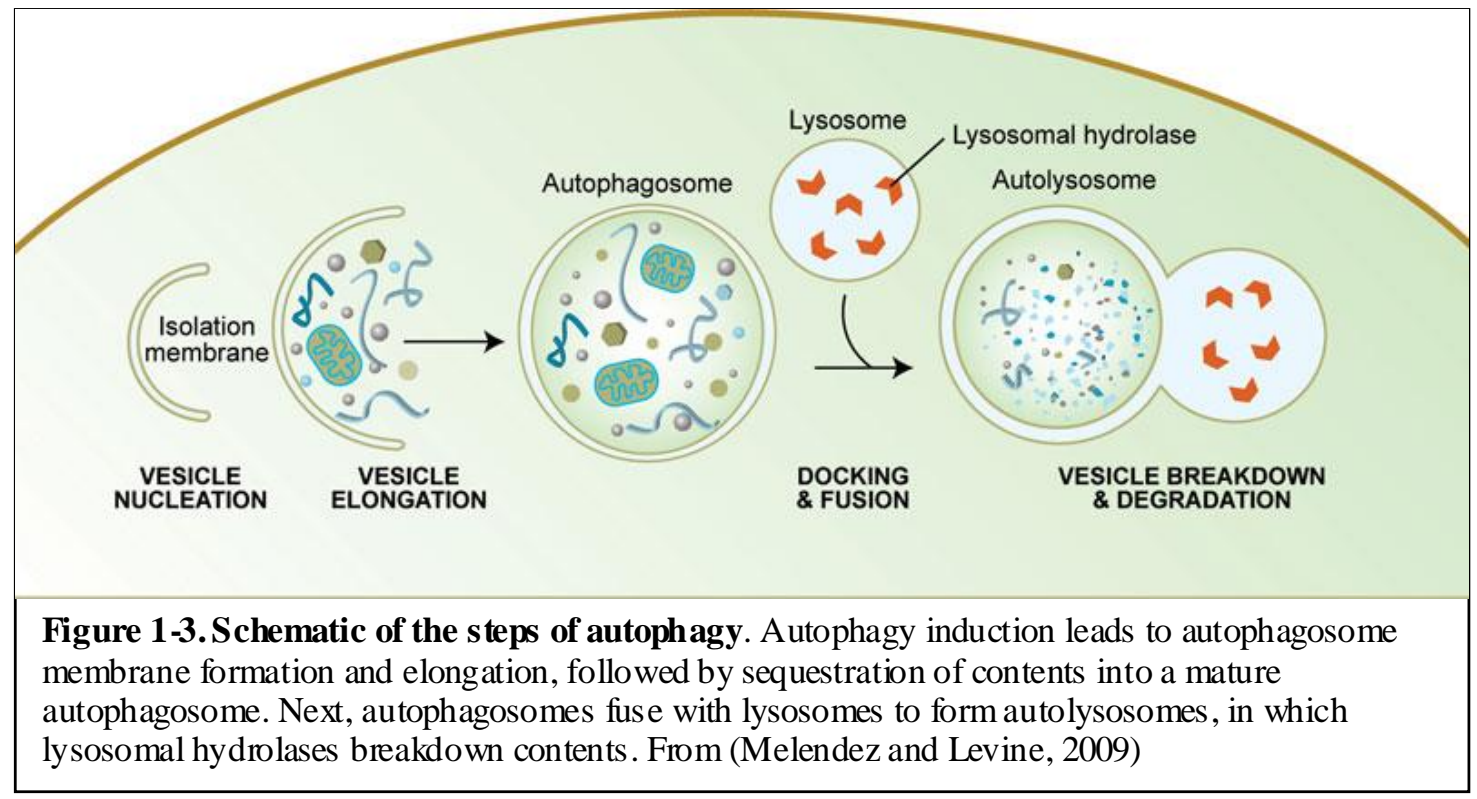

Autophagosomes are membrane-bound vesicles with a double membrane, us ually containing cytoplas m and/or organelles (Eskelinen, 2008). The mechanism and origin of autophagos ome formation is still relatively unclear. Studies have show $\mathrm{n}$ that autophagos ome membranes could be formed via de novo synthesis or from parts of the endoplasmic reticulum, plasma me mbrane, or even the mitochondrial outer membrane (Dunn, 1990; Hailey et al., 2010; Ravikumar et al., 2010; Tooze and Yoshimori, 2010). During the formation of autophagosomes, microtubule-associated prote in light chain 3 
(LC3) is cleaved to LC3-I and subsequently lipidated with phosphatidylethanola mine to produce the autophagosome membrane-bound LC3-II form (Kabeya et al., 2000).

The nature of autophagosome cargo selection was initially thought to be nonspecific bulk degradation of cytoplasmic contents. The specificity and selectivity of autophagy was clarified when a role for p62/SQSTMI (p62/sequestosome1, from now on referred to as p62) was described (Bjorkoy et al., 2005). Polyubiquitination of substrates is normally a signal for de gradation via the UPS (Chau et al., 1989). However, ubiquitin has also been identified as a marker for selective autophagy. The adaptor prote in p62 can bind both LC3 (Pankiv et al., 2007) and ubiquitin (Kim et al., 2008), leading to the degradation of ubiquitinated substrates via autophagy.

\section{Mitophagy}

Defective mitochondria may be selectively degraded by autophagy, via a process known as mitophagy (Lemasters, 2005). This is an important part of the maintenance of normal mitochondrial function in cells; by eliminating and replacing dysfunctional mitochondria, cells can maintain normal respiratory function. In normal cells, mitochondria exist in cycles of fission and fusion with other mitochondria in the cell. These fission and fusion cycles help the cell maintain a healthy pool of mitochon dria (Mouli et al., 2009). Portions of a mitochondrion can lose normal me mbrane potential as part of the normal mitochondrial life cycle. When this happens, the mitochondrion can undergo fission to isolate the abnormal section of the mitochondrion (Twig et al., 2008b). The product of this fission is two daughter mitochondria, one with a normal membrane potential and a second with an abnormal membrane potential. As part of the normal mitochondrial life cycle, mitochondria with abnormal membrane potential can then fuse 
with another mitochondria to regain normal membrane potential.

Mitochondria that are unable to recover membrane potential, either alone or by fusion, are degraded via a specific form of autophagy, called mitophagy (Figure 1-4) (Lemasters, 2005; Twig et al., 2008a).

As described above, several proteins play important roles in both mitochondrial quality control and autosomal PD. This suggests that mitochondrial and autophagic function may both be important processes for PD pathogenesis. The discovery of two genes that can cause familial PD, PINK1 and parkin, has led to a greater understanding of the proteins involved in mitophagy. Knockdown of these

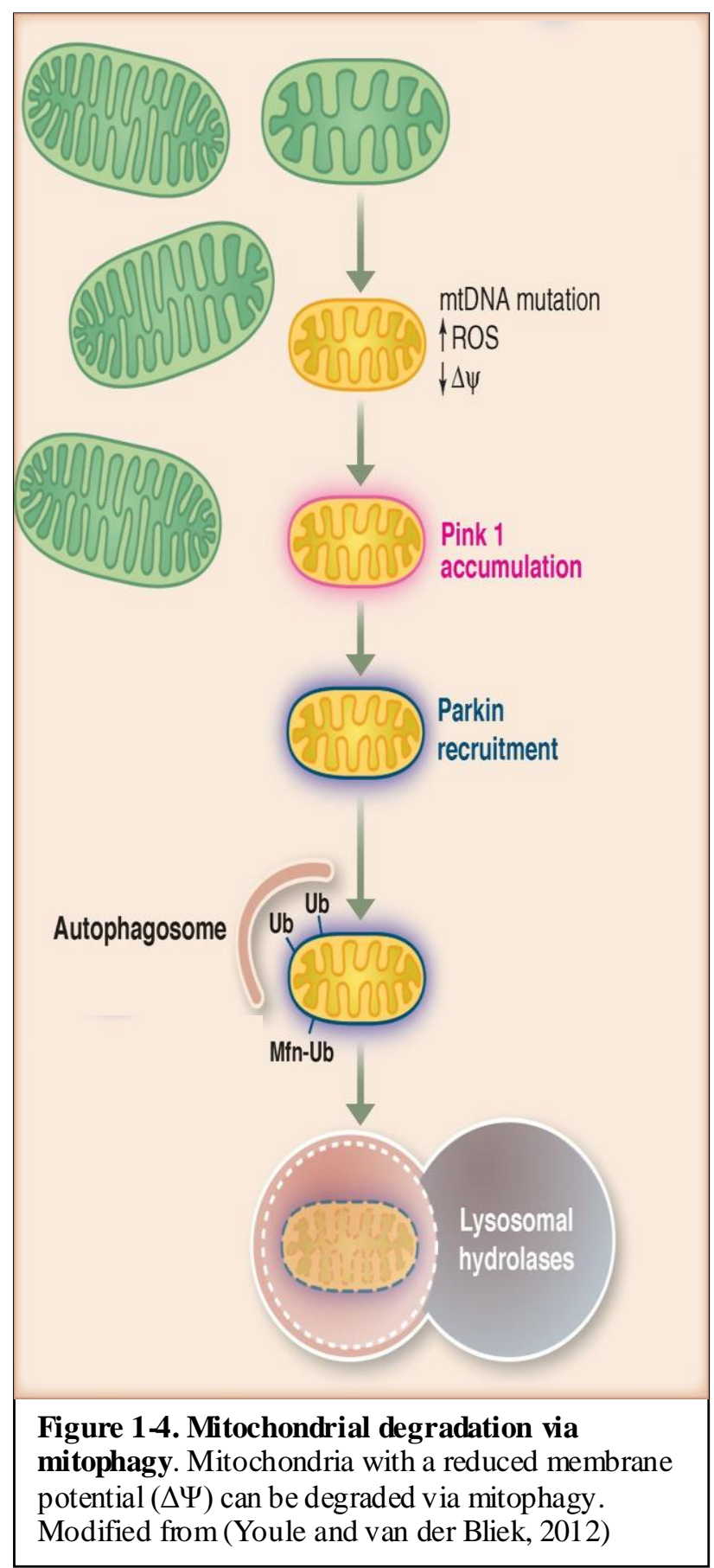

proteins in mouse and Drosophila models caused the accumulation of abnormal mitochondrial morphologies, loss of mitochondrial respiratory capacity, and increased production of free radicals (Palacino et al., 2004; Park et al., 2006). A stable PINK1 knockdown in SH-SY5Y cells also produced abnormal mitochondrial morphology and an 
increase in number of autophagosomes, suggesting an involvement of PINK1 in both mitochondrial function and autophagy (Dagda et al., 2009). In light of these findings, a role was established for both PINK1 and parkin in mitochondrial quality controls. Narendra et al found that PINK1 is involved in the recruitment of parkin to de polarized mitochondria that are subsequently degraded via mitophagy (Narendra et al., 2008). Mitophagy is important quality control mechanism for the maintenance of a health $y$ cellular pool of mitochondria. As the studies described above show, a loss of mitochondrial quality control can lead to a number of de leterious consequences for cells, such as increased free radical production, decreased ATP levels, abnormal calcium buffering, and potentially the initiation of apoptosis.

Mitochondrial function is also re levant to autophagy, independent of mitophagy. As cells age, misfolded proteins, oxidatively damaged prote ins, and dysfunctional organe lles can accumulate (Terman and Brunk, 2006). Autophagy can be a protective mechanism for the clearance of these misfolded proteins and dysfunctional organelles from the cytoplasm (Lum et al., 2005). The turnover of dysfunctional proteins and organelles is especially important in post-mitotic cells, like neurons, which cannot divide to eliminate damaged cell contents. However, autophagy can also decline as cells age (Martinez-Vicente et al., 2005; P lomp et al., 1989; Plomp et al., 1987). ATP is required for autophagic function at multiple steps, such as during the initiation of autophagosome membrane formation and to maintain the hydrolytic enzymes in lysosomes (Plomp et al., 1989; Plomp et al., 1987). It is important to note that degra dation of proteins via the UPS is also ATP dependent (Armon et al., 1990). Therefore, a decline in mitochondrial function can lead to a decline in the efficiency of both the UPS and autophagy. This can 
have increasingly detrimental effects on cells as mitochondrial turnover is reduced and ATP production declines, leading to a decrease in overall protein proteolysis and increasing amounts of aggregated prote ins and dysfunctional organelles in the cytoplasm. Importantly, we suspect that this loss of autophagic efficiency is likely to be a contributing factor to the formation LB, which contain aggregated proteins and dysfunctional orga nelles.

\section{Lewy bodies}

A definitive pathologic diagnosis of PD requires the presence of LB and Lewy neurites (LN) in the nigra of post-mortem brain (Dauer and Przedborski, 2003). LB are eosinophilic, cytoplasmic inclusions found primarily, but not exclusively, in DA neurons (Figure 4) (Forno, 1996; Pollanen et al., 1993). Ultrastructural analysis reveals that nigral LB have a dense membranous core with filaments that radiate outwards through the halo (Duffy and Tennyson, 1965; Forno, 1996).

This core and halo composition distinguishes LB from the intracellular inclusions found in other diseases (Den Hartog Jager and Bethlem, 1960). LB found in the cortex and other extranigral areas lack the typical core and halo composition observed in nigral LB (Forno, 1996). The insolubility of LB ruled out an initial assumption that the main fibrillar components were neurofilaments (Galloway et al., 1992). After the

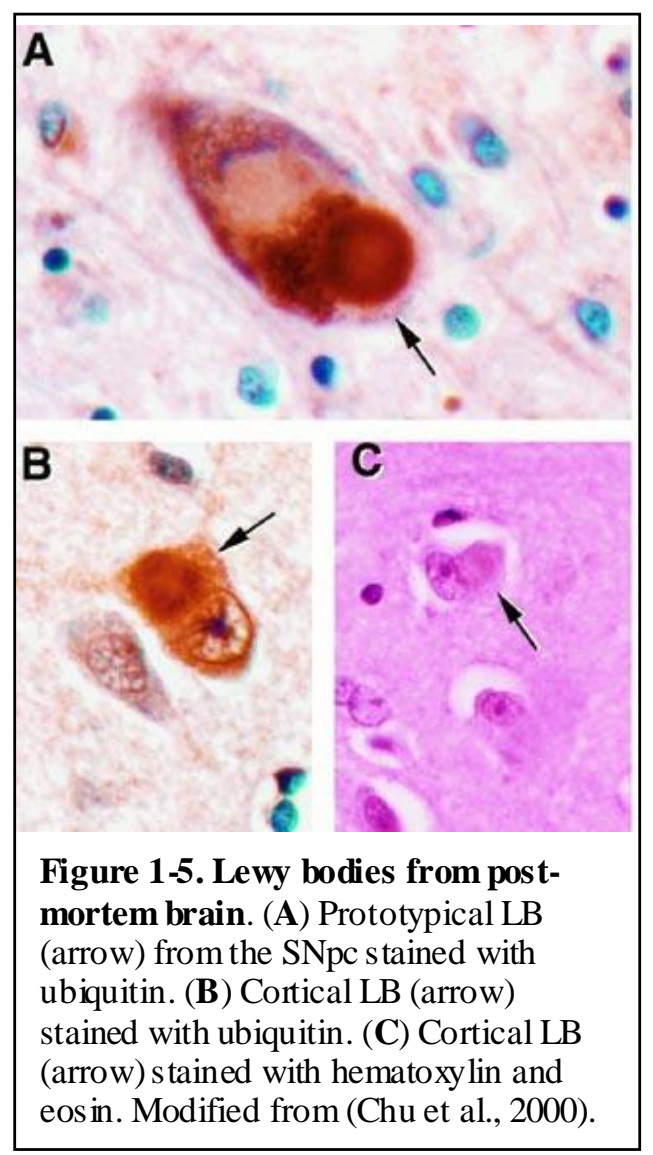


discovery of the SNCA gene in familial PD, aSYN was identified as the fibrillar component of LB (Galloway et al., 1992; Spillantini et al., 1997). It has been suggested that cross-linking between filaments could be responsible for the composition and insolubility of LB (Castellani et al., 1996). This implicates oxidative reagents, such as free radicals, which are known to induce cross-linkages in aSYN (Shaikh and Nichols on, 2008). In addition to aSYN, many of the other components identified in LB are involved in processes such as aSYN binding, the UPS, autophagy, and cytoskeletal maintenance (Wakabayashi et al., 2007).

Aggregation can also occur within the neurite, resulting in dystrophic neurites or LN (Braak et al., 2003). These LN are normally found in axons and it is unknown if they can form in dendrites (Braak et al., 2001). According to the Braak staging, LN may occur before LB formation during PD progression and it has been suggested that this is a better correlate with disease progression than LB (Del Tre dici et al., 2002).

The presence of LB occurs in association with the cell loss, especially in the dopaminergic, melanin-containing cells of SNpc (Hirsch et al., 1988). However, analys is of post-mortem brain shows that neurons containing LBs do not have a greater susceptibility to undergoing apoptosis than non-LB containing cells (Tompkins and Hill, 1997). This suggests that the formation of LB is not necessarily detrimental to the cell. In fact, the purpose of LB formation has been hypothesized to be a cytoprotective cellular mechanism, although this remains controversial (Harrower et al., 2005).

Although the structure of aSYN within a nigral LB is believed to be fibrillar and inert, they may also contain oligomeric aSYN (Fagerqvist et al., 2013). Studies have suggested that it is the oligomeric and pre-fibrillar (protofibril) forms of aSYN aggregates 
are more toxic to the cell than the fibrillar aSYN in a LB (Ding et al., 2002; Winner et al., 2011). Therefore, it is reasonable to propose that sequestration of protofibrils forms into LBs, where they can mature into an inert fibril formation, may be neuroprotective (Bodner et al., 2006). It is likely that in order to isolate the protofibril forms from the cytoplas m, cells must have the required resources, in particular ATP, to drive this process. This would suggest that only cells with sufficient mitochondrial function would be capable of generating a LB. LB could also contribute to neuroprotection by serving as a site of degradation of aggregated proteins (See Chapter 5). This could explain the presence of several autophagy and UPS related prote ins in LB (Alvarez-Erviti et al., 2010; Wakabayashi et al., 2007; Zatloukal et al., 2002). The controversy over the nature of LB as protective or detrimental to cells is something we are uniquely able to address us ing our model, which exhibits both mitochondrial dysfunction and LB formation. Studies addressing this hypothesis are described in Chapter 3. Are Lewy bodies a sign of a breakdown in proteolysis?

Due to the accumulation of ubiquitinated proteins in LB in PD (Forno, 1996), the involve ment of the UPS in PD pathogenesis has been widely investigated. The ubiquitin hydrolase enzyme UCH-L1 is mutated in a rare form of familial PD, suggesting a possible link between UPS dysfunction and the development of PD (Leroy et al., 1998). Furthermore, proteasomal degradation of the A53T form of aSYN is reduced compared to wild-type aSYN (Bennett et al., 1999). Reduced proteasomal degradation has also been reported in the SNpc of idiopathic PD patients compared to controls (McNaught and Jenner, 2001). Although these findings suggest a role for UPS in LB formation in PD, 
other studies have reported that degradation of both monomeric and aggre gated aSYN may not be proteasome dependent (Ancolio et al., 2000).

In addition to the UPS, the involvement of autophagy in PD has been well established in the literature. Proteins involved in autophagy colocalize with markers of LB (Alvarez-Erviti et al., 2010). For example, p62 has been observed in protein aggregates found in several different diseases, including LB found in PD brain (Zatloukal et al., 2002). Neurons undergoing autophagic degradation have also been observed in the nigra of PD patients (Anglade et al., 1997). Altered LC3 expression has been found in post-mortem bra in of Dementia with Lewy bodies patients as well, suggesting that the role of autophagy in aggregate formation is common to other ne urological conditions with protein aggregation (Higashi et al., 2011; Tanji et al., 2011).

Several in vitro studies have investigated the specific interactions between aSYN and autophagy. CMA was shown to degrade wild type aSYN, yet the mutated forms of aSYN block CMA, potentially resulting in an upregulation of non-CMA autophagy to compensate for the reduced CMA activity (Cuervo et al., 2004). Aggre gated wild-type aSYN was shown to inhibit autophagy, leading to a decrease in LC3-II levels (Winslow et al., 2010). Artific ially aggregated or ectopically expressed aSYN can be sequestered into autophagosomes and cleared by p62-dependent autophagy (Watanabe et al., 2012; Wong et al., 2008). Conversely, pre-formed aSYN fibrils were not degradable by autophagy, even though the fibrils colocalized with p62, LC3, and lysosomal proteins (Tanik et al., 2013). Based on these studies it's reasonable to suggest that a relationship exists between autophagy, aSYN, and LB formation, however the nature of this 
relationship is unclear and warrants further study. This topic will discussed further in Chapter 5.

The studies described above clearly indicate a role for autophagy in aSYN degradation and, in some cases, a loss of autophagic efficiency when aSYN is aggregated (Tanik et al., 2013; Winslow et al., 2010). Current studies in the literature have also shown that inhibition of autophagy can lead to aggregate formation if mutant aSYN is also over-expressed (Klucken et al., 2012). Over-expression models commonly used to determine the role of aSYN in LB formation are unsatisfactory. These models are less relevant to sporadic PD, in which aSYN is not over-expressed. Furthermore, excessive overexpression could trigger an exaggerated response. It is also important to note that many cell culture models use non-neuronal cells, such as HeLa or HEK292 cells (Tanik et al., 2013; Watanabe et al., 2012; Winslow et al., 2010) The advantage of the cybrid model is that PD cybrid cells spontaneously form LB without overexpression of aSYN, pharmacological inhibition, or gDNA manipulation. As described in the following chapters, we used this model to investigate the relationships between mitochondrial dysfunction and aggregate formation for insights into the ir roles in PD pathogenesis. Cybrid model

The overwhelming majority of cell and animal models of PD have focused on the familial mutations present in a small majority of patients $(<10 \%)$ or the mitochondrial toxins that can cause parkinsonis $m$ in patients. None of these models have successfully recapitulated the features of the disease nor have they led to the successful development of new therapeutics (Blandini and Armentero, 2012). In particular, most cell models fail to create LB even with pharmacological or genetic manipulation (Trimmer and Bennett, 


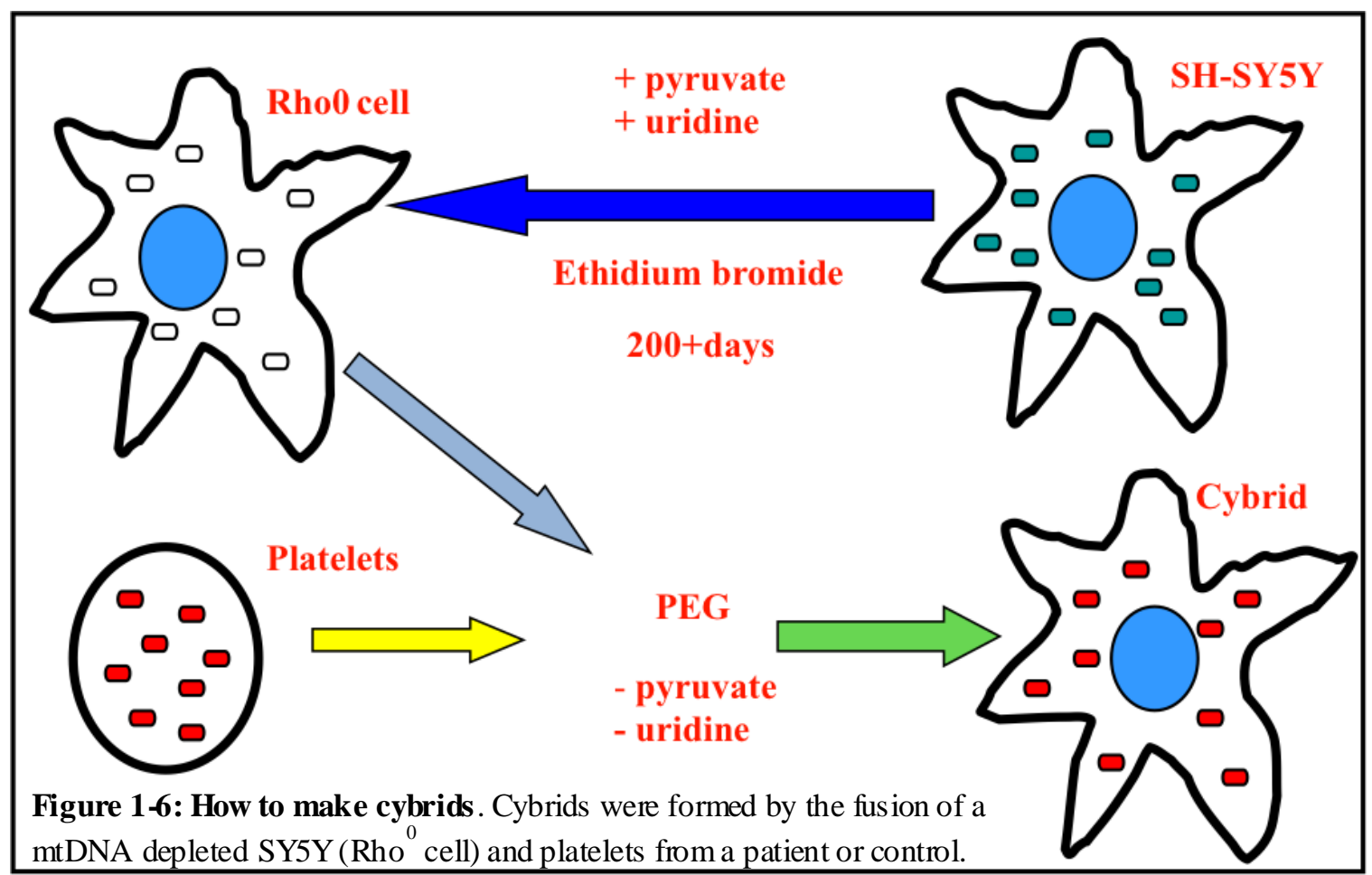

2009). In light of the known mitochondrial dysfunction and mtDNA damage observed in

both the central and peripheral neurons in PD patients, a cell model of sporadic PD was created using mitochondrial DNA from patients (Trimmer and Bennett, 2009). These cell lines are cytoplasmic hybrids (cybrids) that result from the fusion of platelets from PD patients and a mtDNA-depleted $\left(\mathrm{Rho}^{0}\right)$ human neuroblastoma cell line, SH-SY 5Y (Figure 6). Rho0 cells were produced by treating SH-SY5Y cells with a low-dose ethidium bromide for an extended period of time. This treatment destroys mtDNA but leaves histone coated nuclear DNA intact (Miller et al., 1996). Platelets from PD patients were used for the source of mtDNA because they do not contain gDNA. Therefore, the resulting cybrid cells contain mtDNA from PD patients with a SH-SY5Y nuclear background (Swerdlow et al., 1996). Importantly, this cell line recapitulates the mitochondrial dysfunction observed in PD patients (Borland et al., 2009; Keeney et al., 2009; Swerdlow et al., 1996). 
One of the major drawbacks to this model is that it was created in a tumor cell line background. The SH-SY5Y cells were cloned from the original SK-N-SH cell line derived from the bone marrow of a female patient with neuroblastoma (Biedler et al., 1973). These cells are not neurons and although they can be differentiated with staurosporine or retinoic acid (Borland et al., 2008; Jala va et al., 1992; Pahlman et al., 1984). Under normal growth conditions, however, they are not post-mitotic. Although this is an important limitation, the cybrid model has several advantages over other models of PD. One of the main critic is ms of animal and cell models that use the gDNA mutations observed in familial PD is that they have failed to produce any therapeutics that are successful in clinical trials (Waldmeier et al., 2006). The failure of these models to accurately reproduce the diversity of PD patients may be the reason many clinical trials have not been successful. As mentioned earlier, the PD patient population is incredibly heterogeneous and most patients do not have the familial mutations used in the models to test therapeutics. This is one of the main advantages of the PD cybrid cell lines; they are derived from sporadic PD patients and recapitulate the diversity found in the se patients (Trimmer and Bennett, 2009).

The other advantage of the PD cybrid cell lines is that these cells lines spontaneously produce intracellular prote in aggregates that are structurally similar to LB observed in PD patients (Trimmer et al., 2004). These inclusions, called cybrid LB (CLB), are positive for the same prote in markers used to identify LB in PD patients, such as aSYN, ubiquitin, lysosomes, thioflavin, and Congo red (Trimmer et al., 2004). The formation of CLB is a directly related to the expression of mtDNA from PD patients in cybrids, as cybrid cells created with mtDNA of control patients very rare ly form CLB 
(Trimmer and Bennett, 2009). CLB rarely exhibit a core and halo and are closer in structure to the LB found in the cortex than to LB found in the SNpc (Trimmer et al, 2004). PD cybrids from individual patients are characterized by mitochondrial dysfunction, exhibit prote in aggregation and generate CLB (Trimmer et al., 2004). These attributes make them an ideal model to study the roles mitochondrial dysfunction, the accumulation of aggregated proteins and protein degradation systems like autophagy play in PD pathogenesis.

\section{Summary}

Mitochondrial dysfunction has been observed in PD post-mortem brain and the consequences of this dysfunction, such as oxidative stress and reduced ATP production, could be responsible for the accumulation and aggregation of aSYN. Autophagy plays an important role in the degradation of cytoplasmic contents, including aggregated aSYN and dysfunctional mitochondria. The current literature suggests that the interaction between mitochondrial dysfunction and the accumulation of aggregated proteins plays an important role in PD pathogenesis. The studies described in this dissertation seek to elucidate this interaction in the context of the PD cybrid model. In Chapter 3, to investigate if increasing CLB expression is linked to mitochondrial dysfunction, we cloned PD cybrid cells for the presence of CLB and measured changes in mitochondrial function, biogenesis and gene expression. We found that the presence of CLB did not predict the mitochondrial respiratory function in the cloned cell lines. In Chapter 4, we expressed a yeast CI protein in a PD cybrid cell line and the SH-SY5Y parent cell line to determine the effect of CI function on protein aggregation and CLB formation. We found that expression of this prote in was sufficient to improve mitochondrial respiratory 
function but did not prevent CLB formation. In light of the findings of the first two studies, we investigated if autophagy was the unifying pathway between mitochondrial function and CLB formation. For Chapter 5, we explored the role of autophagy in mitochondrial quality control and CLB formation in the PD cybrid cells. We found that autophagy is necessary for maintenance of mitochondrial respiratory function and is clearly involved in the trafficking of prote ins to CLB. These studies taken together show the importance of the interactions between mitochondrial function and protein degradation in PD pathogenesis. 


\section{General Methods}

\section{Cybrid cell line culture}

PD and control cybrid cell lines were created from individual patients and spouse controls (Trimmer et al., 2004). All subjects consented to participate in the IRB approved study at the University of Virginia (Table 2-1). For the generation of cybrids, SH-SY5Y cells were treated with $5 \mu \mathrm{g} / \mathrm{mL}$ ethidium bromide to destroy mtDNA, creating the rho ${ }^{0}$ cell line, which has the SH-SY5Y nuclear background (Figure 1-6, blue arrow) (Miller et al., 1996). Using polyethylene glycol, rho ${ }^{0}$ cells were fused with platelets from PD patients or controls, to introduce mtDNA from patients or controls (Figure 1-6, green arrow). Cells that had undergone successful fusions were selected by staining for mtDNA with PicoGreen (Life Technologies) and individual cell lines were expanded and frozen as stocks in liquid nitrogen for future use (Swerdlow et al., 1996).

Table 2-1. PD patient and control demographics

\begin{tabular}{|c|c|c|c|c|}
\hline $\begin{array}{c}\text { Cybrid } \\
\#\end{array}$ & Age & Gender & $\begin{array}{c}\text { PD } \\
\text { duration }\end{array}$ & $\begin{array}{l}\text { H\&Y } \\
\text { stage }\end{array}$ \\
\hline 61 & 65 & M & $15 \mathrm{yrs}$ & 2 \\
\hline 63 & 73 & $\mathrm{~F}$ & 14 yrs & 2 \\
\hline 65 & 69 & M & $18 \mathrm{yrs}$ & 2 \\
\hline 66 & 75 & M & $7 \mathrm{yrs}$ & 2 \\
\hline 67 & 72 & $\mathrm{M}$ & $8 \mathrm{yrs}$ & 2 \\
\hline 56 & 72 & M & CTRL & \\
\hline 64 & 60 & $\mathrm{~F}$ & CTRL & \\
\hline 68 & 69 & M & CTRL & \\
\hline 91 & 61 & $\mathrm{~F}$ & CTRL & \\
\hline
\end{tabular}

For the experiments described in this dissertation, both PD and control cybrid cells were grown in growth media (GM), composed of Dulbecco's modified Eagle medium (DMEM) with high glucose

(Gibco) with $10 \%$ fetal bovine serum (FBS, Hyclone), $100 \mu \mathrm{g} / \mathrm{mL}$ pyruvate, $50 \mu \mathrm{g} / \mathrm{mL}$ uridine, and antimycotic/antibiotic (Gibco), in $5 \% \mathrm{CO}_{2} / 95 \%$ room air at $37^{\circ} \mathrm{C}$. Cells were 
most of ten grown in T75 $\mathrm{cm}^{3}$ flasks (Greiner B io-one). Cells were only kept in culture for 2 months intervals, to avoid mycoplasma contamination, before fresh stocks were thawed from liquid nitrogen.

For Chapter 3, PD cybrid cell lines used were PD61, PD63, and P67, and then controls used were CTRL56, CTRL64, and CTRL68. For Chapter 4, PD61 and SH-SY5Y cell lines were used. In Chapter 5, PD61, PD63, PD65, PD66, and PD67, were used, along with CTLR56, CTRL64, CTRL68, and CTRL91. Unique culture conditions are described in the Methods section of each chapter.

\section{Immunocytochemistry}

For immunocytochemistry experiments, cells were grown in $35 \mathrm{~mm}$ poly-lysine coated dishes with coverslip \#0 (MakTek Corp) for 48-72 hours to 70-80\% confluency. Cells were then fixed in $4 \%$ paraformaldehyde. At this point, one of two protocols was used. For staining of aSYN (1:400, Chemicon), ubiquitin (1:200, Enzo), LC3 (1:200, Novus Biologicals), LAMP2A (1:200, BD Biosiences), p62 (1:200, BD Biosciences), and Ndi1 1:600 (Barber-Singh et al., 2010) cells were permeabilized using 10mM sodium citrate buffer with $0.05 \%$ Tween for 20 minutes at $95^{\circ} \mathrm{C}$. Cells were then blocked in $0.5 \% \mathrm{BSA} / 0.5 \%$ Triton blocking buffer for 1 hour at room temperature. Primary antibodies were diluted in a 1:10 dilution of blocking buffer and dishes were incubated overnight at $4^{\circ} \mathrm{C}$. Fluorophore tagged secondary antibodies (AlexaFluor 488, 568, or 633, 1:400, Life Technologies) were also made up in a 1:10 dilution of blocking buffer. Dishes were incubated in secondary antibody at room temperature for 2 hours. Vectashield with DAPI (Vector Labs) was added as a mounting medium and a nuclear counter stain. 
For staining using mitochondrial antibodies (porin, CV-a, both 1:400, Mitosciences), a different protocol was used, according to manufacturer's instructions (Mitosciences, Abcam). Cells were treated first in antigen retrie val buffer (urea buffer, $\mathrm{pH} 9.5)$ at $95^{\circ} \mathrm{C}$ for 20 minutes and then permeabilized in $0.2 \%$ triton for 15 minutes at room temperature. Dishes were blocked in $10 \%$ goat serum. Primary antibodies were diluted in the blocking buffer, and dishes were incubated in primary antibody overnight at $4^{\circ} \mathrm{C}$. Cells were stained with fluorescent secondary antibodies (TexasRed, 1:200, Mitoscience) in diluted in $1 \%$ goat serum for 2 hours at room temperature before Vectashield plus DAPI was added to dishes. All images were collected using an Olympus FV300 or FV1000 laser scanning confocal microscope.

\section{Immunoblotting}

For immunoblotting, cells were grown in $\mathrm{T} 175 \mathrm{~cm}^{2}$ flasks (Greiner) to $80-90 \%$ confluency and harvested in $1 \mathrm{X}$ radioimmunoprecipitation assay buffer (RIPA) with protease inhibitors and phenylmethanesulfonylfluoride as previously described (Keeney et al., 2009). The soluble fraction was isolated by centrifugation. Soluble prote in quantity was measured using a Micro BCA kit (Pierce). Equal amounts of protein were loaded on to Bis-Tris gels (Bio-Rad) and run using the Bio-Rad Criterion system. Proteins were transferred onto nitrocellulose membranes using the $\mathrm{iB}$ lot transfer system (Life Technologies). Membranes were blocked using Li-Cor blocking buffer and stained with primary antibodies at room temperature (Ndi1: 1:2000, (Barber-Singh et al., 2010) or MitoProfile Total OXPHOS cocktail: 1:400, Mitosciences). Membranes were then washed and stained with infrared secondary antibodies at room temperature $(1: 4000, \mathrm{Li}-$ Cor). Membranes were imaged using the Odyssey scanner (Li-Cor). Band densities were 
calculated as integrated intensities using the Odyssey software. Integrated intensities were normalized to porin (1:2000 Mitosciences) or actin (1:2000, Sigma).

\section{Quantitative Real-Time Polymerase Chain Reaction (qRT-PCR)}

Cell pellets of approximately $10 \times 10^{6}$ cells were collected from sub-confluent T175 $\mathrm{cm}^{2}$ flasks. RNA and genomic DNA were isolated using an RNA/DNA isolation kit (Qiagen). RNA and gDNA quantity was measured using a NanoDrop (Thermo). From RNA, complementary DNA (cDNA) was made using the iScript cDNA synthes is kit (Bio-Rad). qRT-PCR was run for single genes (EvaGreen Power mix, Bio-Rad) or in a multiplex set (iQ Multiplex Power mix, B io-Rad) in a CFX96 Real-Time PCR Detection System (Bio-Rad). Bio-Rad CFX Manager software calculated starting quantities for samples, based on the standard curve from known starting quantities. For Chapter 3, values were normalized to the genomic mean of three endogenous references genes: 18s RNA, glyceraldehyde 3-phos phate dehydrogenase, and $\beta$-actin. For Chapter 4, values were normalized to endogenous reference genes based on geNorm (BioGazelle) analysis to find the genes with the highest expression stability. For complementary DNA, we used glyceraldehyde 3-phosphate dehydrogenase and 14-3-3-z and for gDNA we used 14-3-3-z and $\beta 2$-microglobulin as the endogenous reference genes.

\section{Live Cell Staining and Analysis}

Cells were plated in $35 \mathrm{~mm}$ dishes as described above and grown for 2-4 days until at least $75 \%$ confluent. Cells were stained with Congo red at $100 \square \mathrm{M}$ for 24 hours. Dishes were then washed two times with clear GM with 25mM Hepes (Gibc o, Life Technologies). Dishes were blinded for image collection and quantification. Images were acquired using an Olympus FV1000 confocal microscope (60X objective) at room 
temperature. Ten representative fields were taken at random per dish and analyze d using MetaMorph image analysis software (Molecular Devices). Studies were repeated with cells from a different passage. Images were set to a common inclusive threshold and pixels over $1 \mu \mathrm{m}$ were measured for total pixel area, pixel intensity and pixel length.

Pixel values were normalized to number of cells in each image. To calculate CLB frequency, Congo red positive inclusions over $2 \square \mathrm{m}$ in diameter were counted for each set of ten images per dish. Number of CLB per dish was normalized to number of cells counted per dish.

\section{Cellular respiration}

Oxygen consumption was measured using the Seahorse Extracellular Flux Analyzer (Seahorse XF24, Seahorse Biosciences) according to manufacturer's instructions (Figure 2-1). In brief, cells were plated in Seahorse XF24 culture plates and grown for 24 hours to form a confluent monolayer. One hour prior to each experiment, growth media was exchanged for unbuffered DMEM, pH 7.4, with $25 \mathrm{mM}$ glucose. The

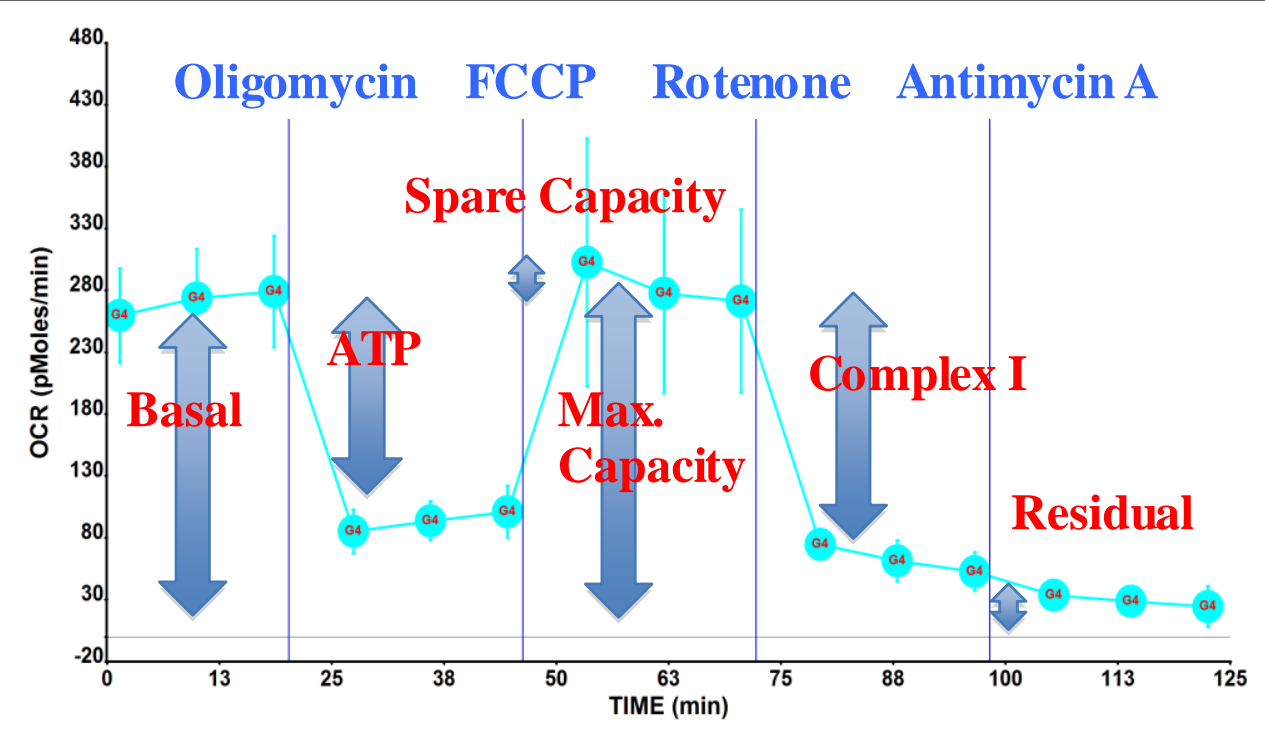

Figure 2-1 Representative trace from Seahorse XF analyzer. Sequential addition of inhibitors are used to examine individual components of the ETC, show by the blue arrows. 
following inhibitors were used to obtain a bioenergetic profile: oligomycin $(1 \square \mathrm{M}), \mathrm{FCCP}$ (carbonyl cyanide 4-(trifluoromethoxy)phenylhydrazone, 300nM), rotenone (100nM), and antimycin $\mathrm{A}(10 \square \mathrm{M})$. For all inhibitors, the $\mathrm{pH}$ was adjusted to 7.4 prior to the experiment. For each Seahorse experiment, three basal measurements of the oxygen consumption rate (OCR) were acquired and calculated by the Seahorse XF. Compounds were added in the order mentioned previously, with two measurements (Chapter 3) or three measurements (Chapters 4 and 5) following each inhibitor. At the end of each experiment, OCR values were normalized to protein content (Micro BCA Kit, Pierce). OCR values are reported as means \pm SEM, except for uncoupled respiration (FCCP), where the highest value was used.

\section{Electron Microscopy (EM)}

Sub-confluent T75 flasks for each cybrid line were fixed with $2 \%$

paraformaldehyde and $2.5 \%$ glutaraldehyde in $0.1 \mathrm{M}$ phosphate buffered saline, processed for EM, sectioned and stained by staff members of the Advanced Microscopy Facility at the University of Virginia, as previously described (Trimmer et al., 2000). Stained sections were imaged on a Jeol JEM-1230 transmission electron microscope at the Virginia Commonwealth University Microscopy Facility.

\section{Statis tical Analysis}

All statistical tests were run using Prism software (GraphPad). Student's t-tests were run when comparing two groups, with We lch's correction for unequal variances used when necessary. Multiple groups were compared using one-way analysis of variance (ANOVA). For all statistical tests, ${ }^{*} \mathrm{p}<0.05,{ }^{*} \mathrm{p}<0.01, \# \mathrm{p}<0.001$.

\section{Mitochondrial quality, dynamics, and functional capacity in Parkinson's disease cybrid cell lines selected for Lewy body expression}




\section{Introduction}

As described in the introduction, the presence of $\mathrm{LB}$ in the $\mathrm{SNpc}$ is one of the neuropathological indicators of PD. The role of LB formation in PD pathogenesis, however, remains unclear. The discovery of mutations in the gene for aSYN (Polymeropoulos et al., 1997), as well as the presence of aggregated aSYN in LB (Spillantini et al., 1997) led to a plethora of models of aSYN aggregation, such as cell and animal models (Blandini and Armentero, 2012; Dauer and Przedborski, 2003). One of the goals of these models was to recreate the LB formation that occurs in PD brain. However, none of these models have successfully recapitulated the formation of LB without using exogenous introduction of aSYN, overexpression of aSYN, or pharmacological inhibition, with the exception of the cybrid model of PD (Trimmer and Bennett, 2009).

In the cybrid model of PD, cells form cybrid LB (CLB) which are perinuc lear, aSYN and ubiquitin positive proteinaceous inclusions, similar to the LB found in PD brain (Trimmer et al., 2004). CLB are formed in PD cybrid cells without overexpression of aSYN or inhibition of proteolytic processes. Mitochondrial dysfunction is however important to CLB formation because introduction of mtDNA from PD patients is sufficient to induce spontaneous CLB formation (Trimmer et al., 2004). Therefore, the PD cybrids are a powerful model that can be used to study the relationships between CLB expression and mitochondrial dysfunction.

The exact relationship between the mitochondrial dysfunction and CLB formation is not fully understood; however, previous studies have show $n$ that the PD cybrids exhibit 
a range of mitochondrial phenotypes, with differences in mitochondrial respiratory function, mitochondrial morphology, oxidative stress, and ETC complex assembly (Keeney et al., 2009; Trimmer et al., 2000). There is also a variation in the frequency of CLB formed by the PD cybrid cells, as not all cells form CLB (Trimmer et al., 2004). The frequency of CLB formation within each PD cybrid cell line is similar to what has been observed in PD brain (3-4\%) (Greffard et al., 2010). Therefore, we wanted to investigate if there is something intrinsically different about the cells that produce CLB compared to the rest of the cells in the PD cybrid cell line. In order to do this, we selected three different CLB-expressing PD cybrid cell lines that exhibit a range in oxygen consumption from severely compromised to near normal function. Each of these three original PD cybrid lines (PD61 $1_{\text {Orig }}$, PD63 $3_{\text {Orig }}$, PD67 ${ }_{\text {Orig }}$ ) was enriched for cells expressing CLB by sub-c loning living cells with Congo red stained CLB to generate cybrid lines PD61 $1_{\mathrm{CLB}}$, PD63 $_{\mathrm{CLB}}$ and PD67 ${ }_{\mathrm{CLB}}$ (see Figure 3-1) (Cronin-Furman et al., 2013).

The effect of LB formation on cell function and survival is not well understood. Several investigators have suggested that LB may be detrimental to neuronal function and survival (Galvin et al., 1999; Lu et al., 2005; Shults, 2006). Studies have also proposed that the sequestration of toxic aSYN into LB is protective (Bodner et al., 2006; Ding et al., 2002). For the purposes of this study, however, we were interested in determining how CLB expression is related to mitochondrial respiratory function and morphology in the PD cybrid cell lines. We antic ipated that CLB-selected PD cybrid lines ( $\left.\mathrm{PD}_{\mathrm{CLB}}\right)$ would exhibit compromised function compared to the original PD cybrid lines ( $\mathrm{PD}_{\text {Orig }}$. Contrary to our expectations, enrichment for CLB expression differentially affected each 
of the three $\mathrm{PD}_{\text {Orig }}$ cybrid lines. Cellular and mitochondrial function improved in PD61 $1_{\mathrm{CLB}}$, worsened in PD63 $3_{\mathrm{CLB}}$ and was unchanged in PD67 ${ }_{\mathrm{CLB}}$. Analysis of our results indicates that CLB expression did not appear to be the driving force for the changes in cellular and mitochondrial function we detected. Rather, the change in function between $\mathrm{PD}_{\text {Orig }}$ and $\mathrm{PD}_{\mathrm{CLB}}$ cybrid lines was determined by the presence or absence of mtDNA in nucleoids in $\mathrm{PD}_{\text {Orig }}$ cells containing CLB.

\section{Methods}

\section{Congo red staining, Cellular respiration, and qRT-PCR}

Methods performed as described in General Methods (Chapter 2).

\section{Ge ne ration of sub-cloned cybrid lines based on CLB expression}

Glass-bottomed 6-well plates (MatTek Corp.) were treated with $200 \mu \mathrm{g} / \mathrm{ml}$ poly-1lys ine $/ \mathrm{H}_{2} \mathrm{O}$ (m.w. 30,000-70,000, Sigma-Aldrich) at room temperature for $\sim 40 \mathrm{~min}$.

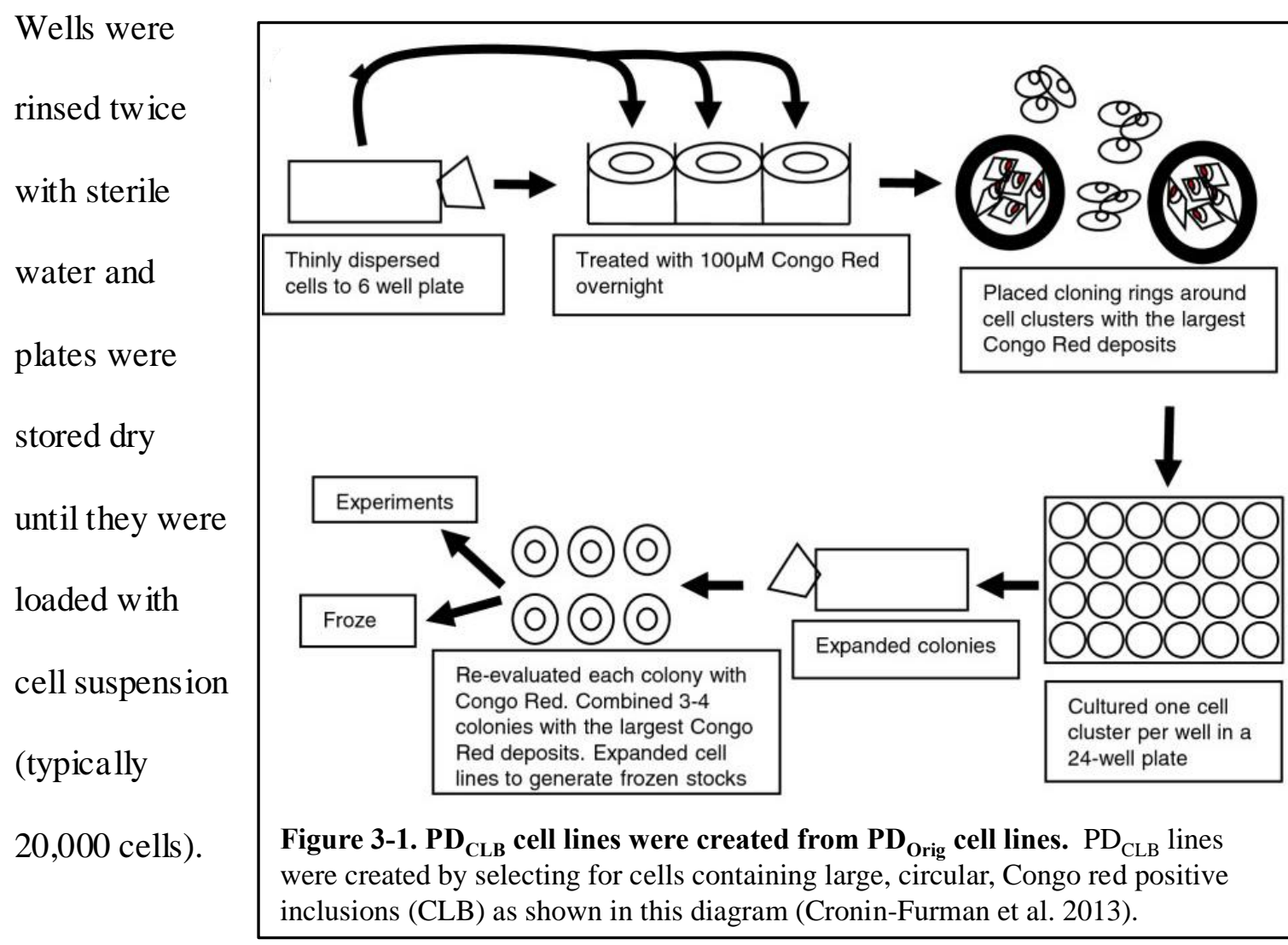


Selected cybrid lines were harvested from T75 CellStar flasks (Greiner bio-one) with 0.05\% tryps in dilute $\mathrm{d}$ in phosphate buffered saline. GM was used to quench trypsin activity prior to re-plating of cells into glass-bottomed 6-well plates (Figure 3-1). Cells were incubated at $37^{\circ} \mathrm{C}$ with $5 \% \mathrm{CO}_{2}$ for $24-48$ hours until cells divided into 2-4 cell clusters. Following an overnight ( 18-24 hours) treatment with $100 \mu \mathrm{M}$ Congo red (Sigma-Aldrich) made up in GM, dishes were rinsed twice with GM without phenol red (clearGM) and further stained with 80nM MitofluorGreen (Life Technologies) in clearGM for 20 minutes at $37^{\circ} \mathrm{C}$. Wells were again rinsed with clearGM and labeled cells were visualized with epi-fluorescence (Olympus IX-70 microscope) using fluorescein is othiocyanate (FITC) filters (Mitofluor Green) and Texas Red filters (Congo red).

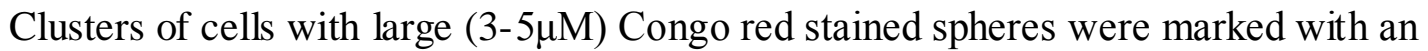
inked objective marker (Olympus) on the underside of the coverslip well (Figure 3-1). After removing GM from the wells, $6 \mathrm{mM}$ sterile glass cloning rings were seated around the marked cell clusters using sterile silic one grease (both from Thermo Fisher). Each cell cluster was trypsinized (see above) and re-plated into one well of a 24-well plate (Figure 3-1). The expression of Congo red positive CLB was later reassessed and the wells with the largest and most numerous Congo red stained spheres were retained and combined (25 clones per well). The other clones were discarded. Combined clones were cultured in GM and passed into larger wells as they became confluent (Figure 3-1). Sub-cloned cybrid lines were expanded into T25 flasks (Greiner bio-one). Each cybrid line was harvested and re-plated into coverslip-bottom dishes and re-selected for CLB expression using Congo red and MitoFluor Green. These colonies were expanded into T75 flasks, at which time aliquots from each line were frozen for subsequent study. 


\section{Nucleoid imaging and quantitation}

Live cells were grown in 35mm Mak-Tek dishes, stained with Quant-It PicoGreen dsDNA ( $3 \mu \mathrm{L} / \mathrm{mL}$ for 2 hours) and MitoTracker Red CMXRos (15nM for 10 minutes) (both from Molecular Probes/Life Technologies) and imaged in clearGM as described above. Dishes were blinded prior to imaging. Six images were acquired at random per dish using an Olympus FV300 confocal microscope. Cell count totals were acquired by counting the PicoGreen positive nuclei per frame. Nucleoids were defined as areas of PicoGreen and Mitotracker Red colocalization. Cells with less than five nucleoids per cell were considered "low/null". All others were considered "high". Only cells with both mitochondria and a nucle us in focus were counted. Two-way ANOVA with Bonferroni multiple comparisons were run to compare $\mathrm{PD}_{\text {Orig }}$ and $\mathrm{PD}_{\mathrm{CLB}}$ cell lines at "low/null" versus "high" (Prism, Graph Pad). For analys is of nucleoids in cells with CLB, cells were co-stained with Congo red, MitoTracker Deep Red (50nM for 45 minutes, Molecular Probes/Life Technologies), and Pic oGreen. Cells were imaged in clearGM on an Olympus FV1000 confocal microscope.

\section{Results}

\section{LB morphology and composition in $\mathrm{PD}_{\text {Orig }}$ and $\mathrm{PD}_{\mathrm{CLB}}$ cybrid cell lines}



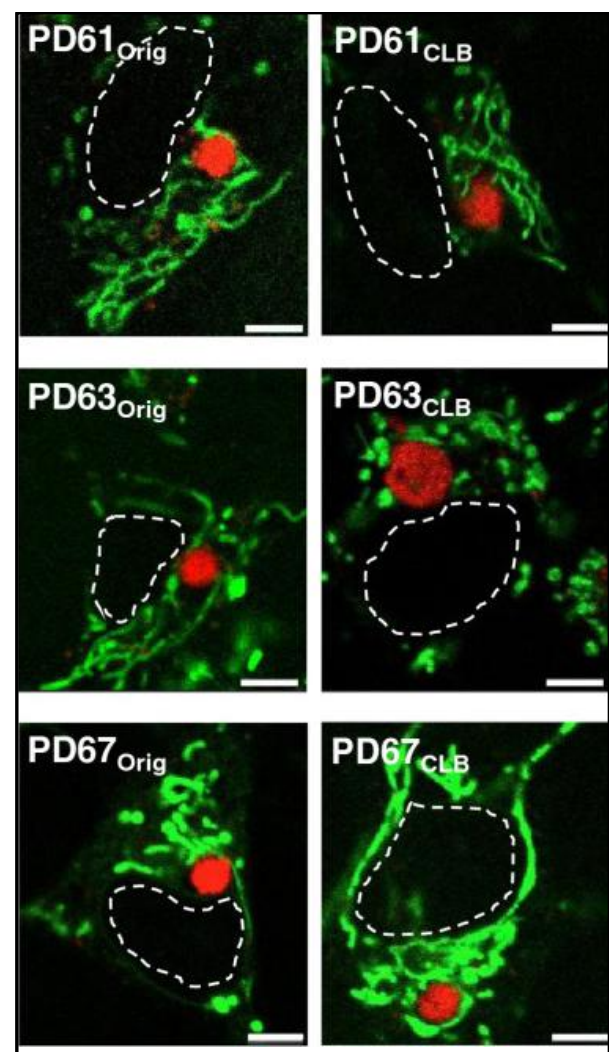

Figure 3-2. Mitochondrial morphology in $\mathrm{PD}_{\text {Orig }}$ and $\mathrm{PD}_{\mathrm{CLB}}$ cybrid cell lines. $\mathrm{PD}_{\text {Orig }}$ and $\mathrm{PD}_{\mathrm{CLB}}$ cybrid cells were stained with Congo red to visualize CLB (red) and MitoTrackerGreenFM to visualize mitochondria (green). The nucleus is indicated by white dashed line. Of the three $\mathrm{PD}_{\text {Orig }}$ and $\mathrm{PD}_{\mathrm{CLB}}$ pairs, only PD63 ${ }_{\mathrm{CLB}}$ had mitochondria that were morphologically different from PD63 Orig. Scale bar $=5 \mu \mathrm{m}$.
Like LB in PD brain tissue, CLB in all

$\mathrm{PD}_{\text {Orig }}$ and $\mathrm{PD}_{\mathrm{CLB}}$ cybrid lines exhibited a consistent range in size and stained uniformly with the histochemical dye Congo red (Figure 3-2). Congo red binds to fibrillar aSYN as well as other misfolded, amyloidal (beta-pleated sheet folded) proteins (Bertrand et al., 2011; Sanchez et al., 2003). While Congo red does not cross the blood brain barrier, it will cross living cell membranes and label intracellular amyloidal aggregates in vitro (Conway et al., 2000). We expected that the $\mathrm{PD}_{\mathrm{CLB}}$ cell lines would show an increase in CLB expression compared to the $\mathrm{PD}_{\text {Orig; }}$, however, assessment of Congo red-positive pixel intensity, pixel length or pixel area revealed no change in CLB expression frequency. There was also no difference in the frequency of Congo red-positive

staining among the $\mathrm{PD}_{\text {Orig }}$ and $\mathrm{PD}_{\mathrm{CLB}}$ lines.

Using electron microscopy, $\mathrm{CLB}$ in all six $\mathrm{PD}_{\text {Orig }}$ and $\mathrm{PD}_{\mathrm{CLB}}$ lines were structurally equivalent (Figure 3-3). The heterogeneous, de nse granular appearance of CLB at the electron microscope le vel (EM) suggests that small protein aggre gates contribute to the continuous formation of CLB (Figure 3-3). LB in PD brain tissue, especially in cortical LB, are also composed of aggregated, dense granular material 
(Dickson, 2012). CLB do not consistently contain straight filaments, consequently they more closely resemble cortical LB, rather than nigral LB (Trimmer et al., 2004).

\section{Mitochondrial morphology in $\mathbf{P D}_{\text {Orig }}$ and $\mathbf{P D}_{\mathrm{CLB}}$ cybrid cell lines}

Mitochondrial changes in shape are intrinsically related to essential cellular functions such as mitochondrial membrane potential, ATP production, calcium signaling and ROS generation (reviewed in Campello and Sc orrano, 2010). Consequently, the morphology of mitochondria either at the light or EM level provides insight into their functional capacity. Using light microscopy, we observed that mitochondria in PD61 Orig, PD63 $3_{\text {Orig }}$ and PD67 $7_{\text {Orig }}$ cells containing CLB varied from elongate to short rod-like or globular in shape (Figure 3-2). The mitochondrial morphology in the PD Orig lines was consistent with previous studies of PD cybrid cell lines (Trimmer et al., 2000).

Mitochondrial morphology at the light microscope level was qualitatively unchanged in PD61 $1_{\text {CLB }}$ and PD67 $7_{\text {CLB }}$ when compared to PD61 $1_{\text {Orig }}$ and PD67 Orig, respectively (Figure 3-2). However the mitochondria in PD63 ${ }_{\text {CLB }}$ were noticeably different from those in PD63 Orig (Figure 3-2). PD63 $3_{\text {CLB }}$ mitochondria were swollen, fragmented and globular, rather than rod-like.

The shift from rod-like mitochondria in PD63 Orig to swollen, fragmented and globular mitochondria in PD63 ${ }_{\text {CLB }}$ is evidence of altered mitochondrial dynamics, such as a change in mitochondrial fission and fusion dynamics. Mitochondrial fragmentation can have many different causes (Knott et al., 2008). Fragmented and dysfunctional, rather than elongated mitochondria, are more susceptible to mitophagy (Gomes et al., 2011; Twig et al., 2008b). Future studies on the activity of mitochondrial fission and fusion prote ins may reveal the mechanism behind mitochondrial fragmentation in PD63 ${ }_{\text {CLB }}$. 
In light of these observations, we also processed fixed pellets of each $\mathrm{PD}_{\text {Orig }}$ and $\mathrm{PD}_{\mathrm{CLB}}$ cell line for EM (Figure 3-3). Mitochondrial morphology at the EM level was qualitatively unchanged in PD67 $\mathrm{CLB}$ when compared to PD67 Orig $($ Figure 2E, F). The
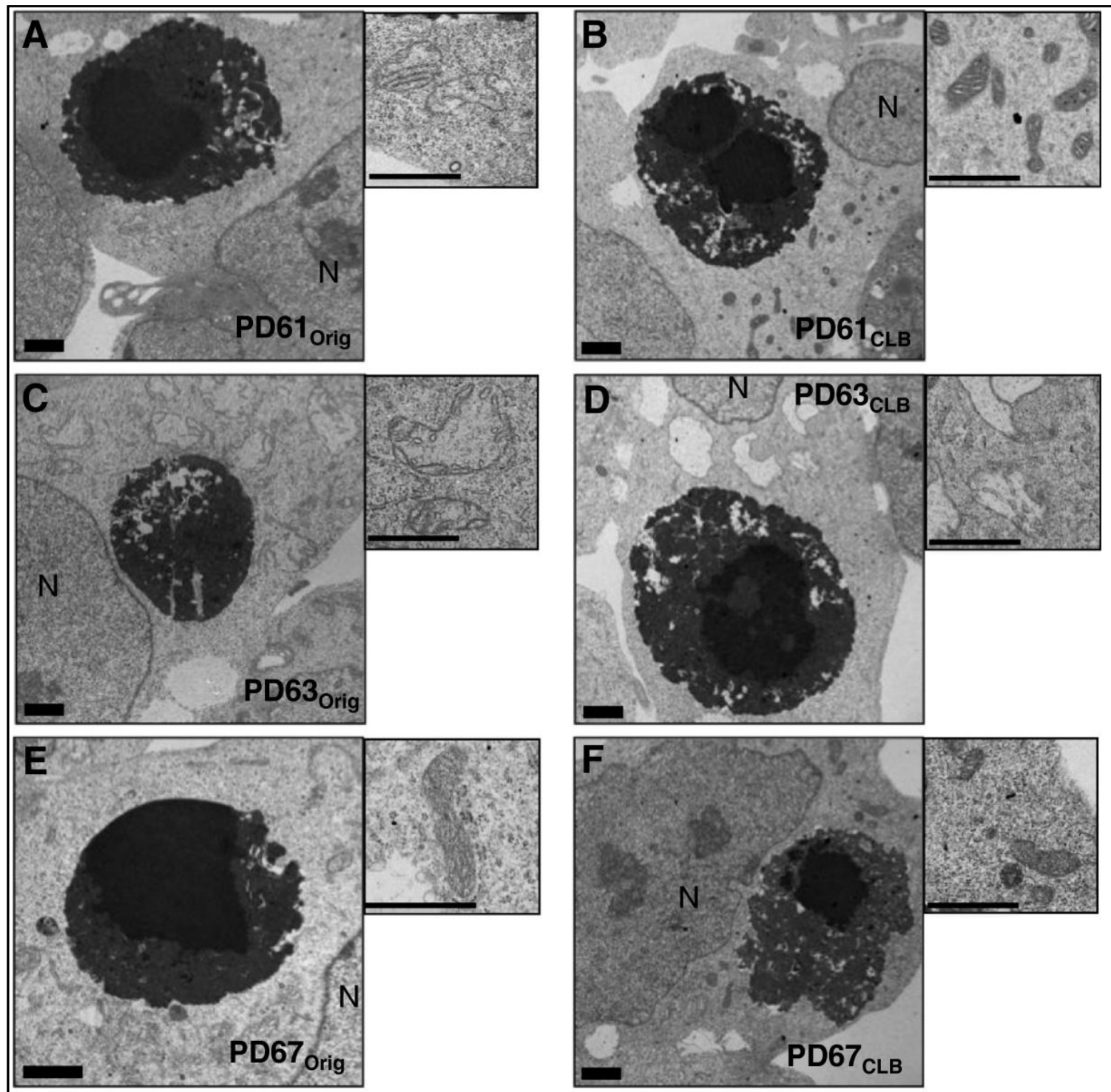

Figure 3-3. Electron micrographs of CLB and mitochondria in $\mathbf{P D}_{\text {Orig }}$ and $\mathbf{P D}_{\mathrm{CLB}}$ cybrid lines. CLB in $\mathrm{PD}_{\text {Orig }}(\mathbf{A}, \mathbf{C}, \mathbf{E})$ and $\mathrm{PD}_{\mathrm{CLB}}(\mathbf{B}, \mathbf{D}, \mathbf{F})$ lines typically had an electron dense, compact core (core in PD63 ${ }_{\mathrm{CLB}}$ is a double). The dense core is surrounded by a halo consisting of electron dense, heterogeneous aggregated material. Paired higher magnification images illustrate mitochondrial morphology in cells containing CLB. (A, B) The mitochondria in PD61 $1_{\text {Orig }}$ were enlarged with reduced numbers of cristae and a pale matrix. Mitochondria in PD61 $1_{\mathrm{CLB}}$ were normal in appearance with a dense matrix and regular cristae. (C, D) Mitochondria in PD63 ${ }_{\mathrm{CLB}}$ were swollen with reduced numbers of fragmented cristae and a little to no matrix while mitochondria in PD63Orig were swollen with fragmented cristae and a pale matrix. (E, F) Mitochondria in PD67 ${ }_{\text {Orig }}$ and PD67 ${ }_{\mathrm{CLB}}$ were rod-like, dense, and featured organized cristae. $\mathrm{N}=$ nucleus. Scale $\mathrm{bar}=1 \mu \mathrm{m}$. 
majority of the mitochondria in both PD67 $7_{\text {Orig }}$ as well as PD67 ${ }_{\text {CLB }}$ cells exhibited normal morphology with a rod-like shape, organized cris tae and a dense matrix (Figure 3-3E, F). At the EM level, mitochondria in PD61 Orig were enlarged (increased width) with a pale matrix and reduced numbers of cristae (Figure 3-3A, B). The mitochondrial morphology in PD61 ${ }_{C L B}$ was improved compared to PD61 Orig $w$ ith normal appearing rod-like mitochondria with a dense matrix and intact cristae (Figure 3-3A, B). The mitochondria in PD63 Orig cells were swollen with a pale matrix and reduced and irregularly shaped cristae (Figure 3-3C, D). PD63 ${ }_{\mathrm{CLB}}$ had primarily globular mitochondria with few cristae and a transparent matrix (Figure 3-3C, D).

\section{Mitochondrial oxygen cons umption in $\mathrm{PD}_{\text {Orig }}$ and $\mathbf{P D}_{\mathrm{CLB}}$ cybrid cell lines}

Taking into account the abnormalities in mitochondrial morphology between some $\mathrm{PD}_{\text {Orig }}$ and $\mathrm{PD}_{\mathrm{CLB}}$ cybrid lines, we measured oxygen consumption using a Seahorse Extracellular Flux Analyzer XF24 (Seahorse Bioscience) (Brand and Nicholls, 2011; Dranka et al., 2011; Ferrick et al., 2008). The three PD $_{\text {Orig }}$ lines that were selected for CLB cloning expressed a range of basal oxygen consumption values prior to cloning (Figure 3-4). If CLB expression is detrimental to cell function, as anticipated, then we anticipated that all three $\mathrm{PD}_{\mathrm{CLB}}$ lines should exhibit reduced oxygen consumption compared to $\mathrm{PD}_{\text {Orig }}$ lines. Figure 3-4 shows the oxygen consumption rates (OCR) of confluent cultures of $\mathrm{PD}_{\text {Orig }}$ and $\mathrm{PD}_{\mathrm{CLB}}$ lines at baseline and after sequential treatment with specific inhibitors (oligomyc in to inhibit ATP synthase, carbonyl cyanide 4(trifluoromethoxy)phenylhydrazone (FCCP) to diss ipate the proton gradient across the inner mitochondrial membrane, rotenone to inhibit CI and antimycin A to in hibit CIII, 
see General Methods). Use of these inhibitors permits the determination of key aspects

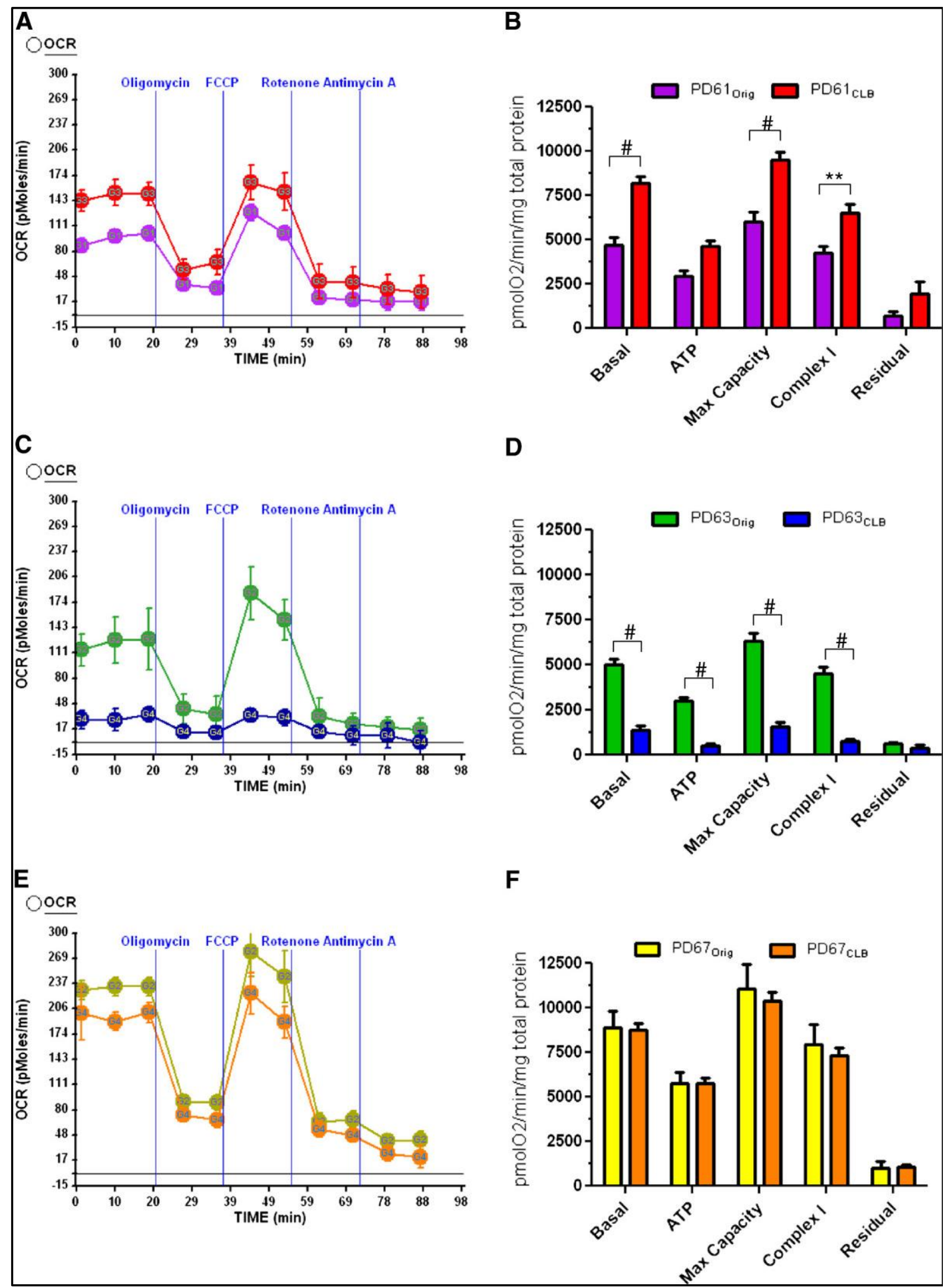


Figure 3-4. Respiration rates of $\mathrm{PD}_{\text {Orig }}$ and $\mathrm{PD}_{\mathrm{CLB}}$ cybrid clones. (A, C, E) OCR was measured using the Seahorse XF24 analyzer for all three PD cybrid pairs and controls (not shown). Oligomycin, FCCP, rotenone, and antimycin $\mathrm{A}$ were added at the same time point for each experiment. in sequential to measure specific components of the ETC. (A, B) PD61 $1_{\mathrm{CLB}}$ had significantly higher OCR but not residual (non-mitochondrial) respiration than PD61 $1_{\text {Orig }}(\mathrm{n}=8)$. (C, D) PD63 ${ }_{\mathrm{CLB}}$ had significantly lower OCR than PD63 $_{\text {Orig }}$ ( $\left.\mathrm{n}=10\right)$. (E, F) OCR in PD67 ${ }_{\mathrm{CLB}}$ did not differ significantly from PD67Orig (n=10). Two-way ANOVA with Bonferroni multiple comparisons; *, p<0.05; **, $\mathrm{p}<0.01 ; \#, \mathrm{p}<0.001$.

of mitochondrial function including basal OCR, maximum capacity OCR, ATP-linked

OCR, CI-linked OCR and the non-mitochondrial (residual) OCR (see Figure 2-1) (Brand and Nicholls, 2011; Dranka et al., 2011). Given the abnormal mitochondrial morphology shown above, it is important to determine if expos ure to specific mitochondrial inhibitors during measurements of OCR cause any cell loss. For all three pairs, there was no difference between $\mathrm{PD}_{\text {Orig }}$ and $\mathrm{PD}_{\mathrm{CLB}}$ clones in cell viability at the end of the Seahorse XF experiment, as measured by live-dead cell counts. The cell viability in these cell lines also did not significantly differ from the disease-free controls $(n=$ three control lines, CTRL56, CTRL64, and CTRL68).

PD61 ${ }_{\mathrm{CLB}}$ had significantly higher basal, maximal and CI-linked OCR when compared to PD61 Orig $($ Figure 3-4A, B) suggesting that sub-cloning of cells expressing CLB resulted in improved oxygen consumption in PD61 ${ }_{C L B}$ cells. This change was specific to the ETC because there was no significant change in glycolysis (measured as extracellular acidification rate- ECAR, a surrogate for lactate production and aerobic glycolysis) or in non-mitochondrial (residual) respiration. Significant improvements in basal, CI-linked and maximum capacity OCR were also cons istent with the improvement in mitochondrial ultrastructure in PD61 ${ }_{\text {CLB }}$ cells compared to PD61 Orig (Figure 3-2).

In contrast, PD63 $3_{\mathrm{CLB}}$ had minimal levels of basal OCR and its response to mitochondrial inhibitors was significantly reduced when compared with PD63 Orig (Figure 3-4C, D). There were also significant reductions in basal, maximum capacity, CI- and 
ATP-linked OCR in PD63 ${ }_{\text {CLB }}$ compared to PD63 ${ }_{\text {Orig. }}$. However, there was no significant change in non-mitochondrial respiration. Therefore, this change in OCR in PD63 ${ }_{\mathrm{CLB}}$ was selective for the mitochondrial ETC. There was also no compensatory up-regulation in ECAR in PD63 $3_{\mathrm{CLB}}$. This was surprising because other studies have shown that loss of CI activity as a result of neurotoxicity induces a loss of OCR with a corresponding increase in ECAR (Dranka et al., 2012). This loss of ETC function and OCR is consistent with the abnormal morphology of mitochondria in PD63 ${ }_{\mathrm{CLB}}$ cells (Figure 3-2).

Finally, PD67 $7_{\mathrm{CLB}}$ exhibited basal, maximum capacity and CI-linked OCR that was unchanged from PD67 $7_{\text {Orig }}$ (Figure 3-4E, F). There was also no change in ECAR (glycolysis) or non-mitochondrial respiration. These OCR values correlate with the cons istent, normal morphology of mitochondria in PD67 Orig and PD67 $7_{\mathrm{CLB}}$ at the light and EM le vels (Figure 3-2, 3-3E, F).

\section{Nucleoid density in $\mathbf{P D}_{\text {Orig }}$ and $\mathbf{P D}_{\mathrm{CLB}}$ cybrid cell lines}

The sub-cloning of cybrid cells expressing CLB did not result in a uniform change in mitochondrial function among the PD cybrid cell line pairs. To establish if changes resulting from sub-cloning could be due to changes in mtDNA distribution, we first visualized nucleoids. Nuc leoids are structures cons isting of one or more mtDNA molecules and associated proteins like single-stranded DNA binding protein, Twinkle, mtDNA helicase and mitochondrial transcription factor A (TFAM) among others (Ashley et al., 2005; Bogenhagen et al., 2008; He et al., 2012). To visualize nucleoids, we used the DNA stain PicoGreen in combination with MitoTracker CMXRos (MTRed; Figures 3-5A, $\mathrm{C}, \mathrm{E}$ ) in live $\mathrm{PD}_{\text {Orig }}$ and $\mathrm{PD}_{\mathrm{CLB}}$ cybrid cells. Nucle oid content was scored as 
"low/null" or "high" in cells from each of the PD cybrid pairs (Figures 3-5B, D, F, see

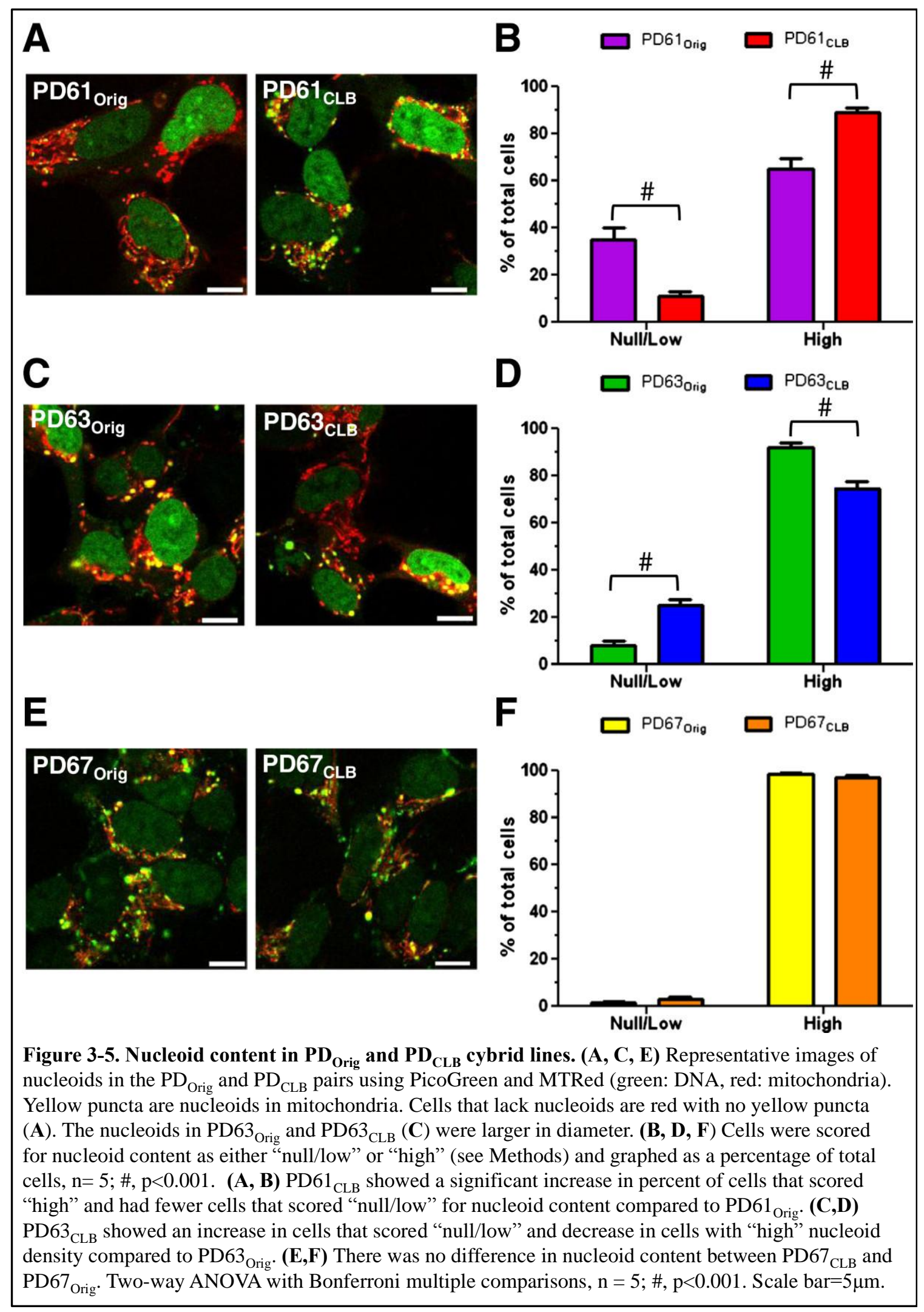


Methods). This is a specific stain for nucleoids, as Rho0 cells that lack mtDNA are devoid of PicoGreen staining and nucle oids (Ashley et al., 2005). PD61 Orig contained cells that fell into the "low/null" category as well as cells with "high" numbers of nuc leoids (Figure 3-5A, B). In contrast, PD61 CLB $_{\text {B }}$ contained significantly fewer cells in the "low/null" category and more cells in the "high" nuc leoid category compared to PD61 Orig $($ Figure 3-5B). This change in cells with "high" numbers of nucleoids is consistent with previous data in this paper showing an improvement in mitochondrial function and morphology in PD61 ${ }_{\mathrm{CLB}}$. PD63 ${ }_{\mathrm{CLB}}$ had significantly more cells that scored "low/null" and fewer cells in the "high" category than PD63 Orig (Figure 3-5D). This was not surprising given the decline in PD63 ${ }_{\mathrm{CLB}}$ mitochondrial function and morphology. Levels of nucleoid expression in PD67 $7_{\text {Orig }}$ and PD67 $7_{\text {CLB }}$ were comparable and consistent with previous data in this paper (Figure 3-5F).

In light of these results, we visualized nucleoids in individual $\mathrm{PD}_{\text {Orig }}$ and $\mathrm{PD}_{\mathrm{CLB}}$ cells containing CLB using fluorescent markers: Congo red (CLB and small prote in aggregates), PicoGreen (nucleoids) and MitoTracker Deep Red (mitochondria) as shown in Figure 3-6. Remarkably, we found that all CLB-positive PD61 Orig cells als o contained numerous nucleoids (Figure 3-6A, top panel). The same result was true of PD67 Orig (Figure 3-6C, top panel). However, the majority of CLB-expressing cells in PD63 Orig did not contain nucleoids (Figure 3-6B, top panel). Taken together, these results indicate that the nucleoid content of the $\mathrm{PD}_{\text {Orig }}$ cells containing CLB correlates with changes in mitochondrial quality and function detected in $\mathrm{PD}_{\mathrm{CLB}}$ cell lines. PD61 $1_{\mathrm{CLB}}$ had better mitochondrial quality and function because it was sub-cloned from PD61 Orig CLBexpressing cells containing numerous nucleoids. PD63 ${ }_{\mathrm{CLB}}$ had reduced mitochondrial 


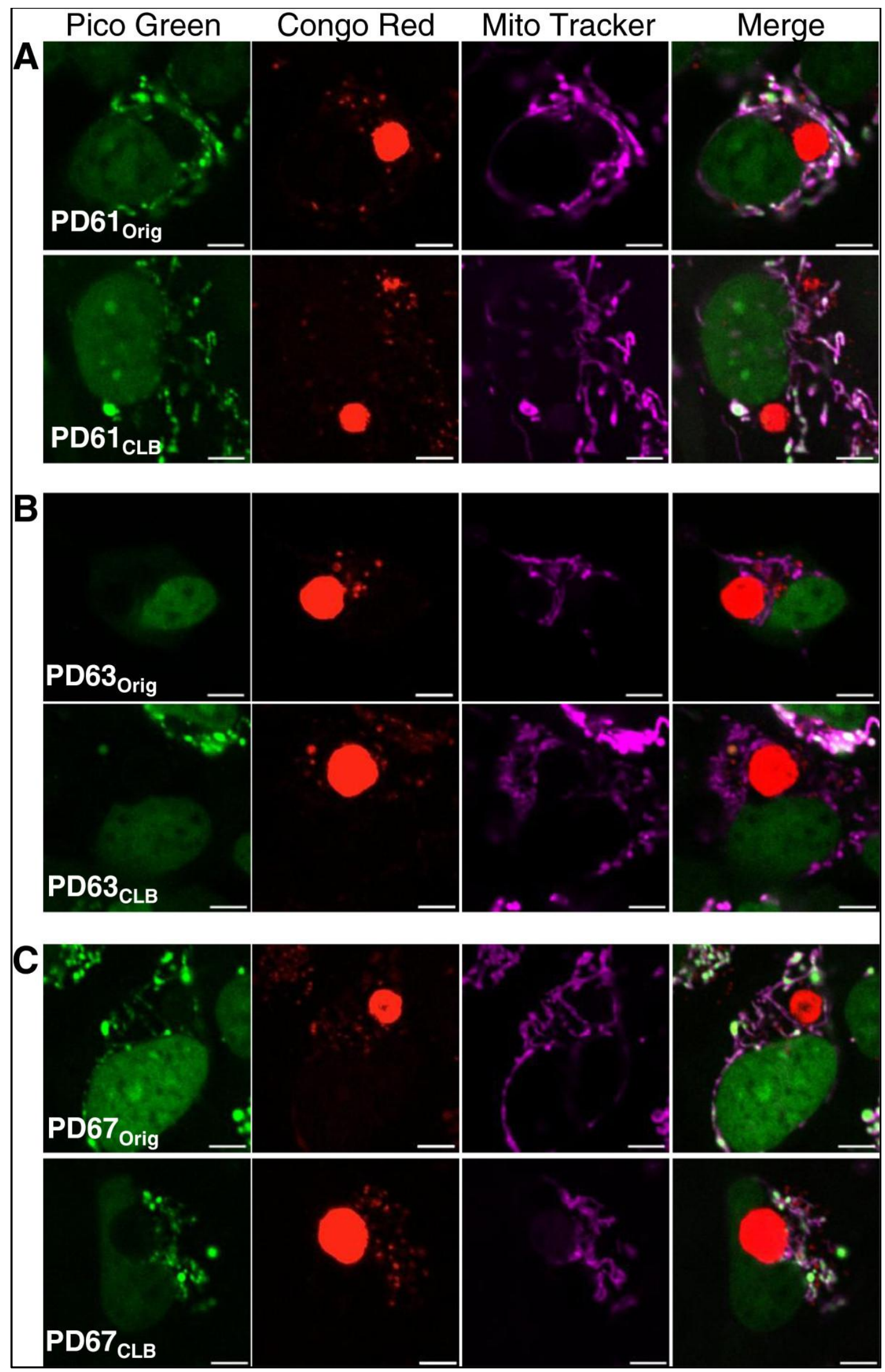


Figure 3-6. Nucleoid content in cells containing $C L B$ in $P_{D_{\text {Orig }}}$ and $P D_{C L B}$ cell lines. $(A, B, C)$ Representative images of cells containing CLB in $\mathrm{PD}_{\text {Orig }}$ and $\mathrm{PD}_{\mathrm{CLB}}$ pairs. Cells were triple-stained with PicoGreen, Congo red, and MitoTracker Deep Red (green: DNA, red: CLB, purple:

mitochondria). (A) CLB-containing cell in PD61 $1_{\text {Orig }}$ contained nucleoids that co-localized with mitochondria. PD61 $1_{\mathrm{CLB}}$ (bottom panel) also featured CLB-containing cells with contained nucleoids. (B) CLB-containing cells from PD63 $3_{\text {Orig }}$ (top panel) and PD63 ${ }_{\mathrm{CLB}}$ (bottom panel) did not contain nucleoids. (C) Cells containing CLB in PD67 ${ }_{\text {Orig }}$ (top panel) and PD67 ${ }_{\text {CLB }}$ (bottom panel) contained nucleoids. Scale bar $=5 \mu \mathrm{m}$.

quality and function because it was sub-c loned from CLB-containing cells in PD63 Orig

with few nucleoids. CLB-containing cells in PD67 $7_{\text {Orig }}$ had numerous nucleoids and these cells yielded the PD67 $\mathrm{CLB}$ cybrid line that also had cells with high numbers of nucleoids as well as mitochondrial quality and function.

Ge ne expression le vels for mitochondrial ETC genes in $\mathrm{PD}_{\text {Orig }}$ and $\mathrm{PD}_{\mathrm{CLB}}$ cybrid cell

\section{lines}

Since nucleoids conta in mtDNA, we examine $\mathrm{mtDNA}$ copy number and gene expression in $\mathrm{PD}_{\text {Orig }}$ and $\mathrm{PD}_{\mathrm{CLB}}$ lines. Previous studies have shown that $\mathrm{CI}$ is damaged and functionally impaired in post-mortem PD cortex homoge nates (Keeney et al., 2006). Analysis of PD cybrid cell lines (including the three PD Orig lines included in the paper) showed that CI gene expression was reduced and showed a robust correlation with the changes in mitochondrialETC gene expression found in post-mortem PD cortex (Borland et al., 2009). Enzymatic dysfunction related to CI assembly is often associated with deficiencies in CIII and CIV because CI assembly intermediates act like a scaffold for the assembly of other complexes in the mitochondrial ETC (Moran et al., 2012). We therefore measured gene expression and gene copy number for mitochondrial genes ND2 and ND4 (CI), CO3 (CIV) and 12s ribosomal RNA using quantitative real-time polymerase chain reaction (RT-qPCR) to create mitochondrial gene expression and copy number profiles (Figure 3-7). 
Figures 7A and B show that mitochondrial gene expression in PD61 $\mathrm{CLB}$ increased nearly 3-fold and copy number increased more than 2-fold over PD61 Orig (Figure 3-7A, B). This observed improvement in mitochondrial gene expression and copy number in PD61 $1_{C L B}$ is consistent with the improved nucleoid content in PD61 ${ }_{\mathrm{CLB}}$ cells. Furthermore, mtDNA copy number improved to control levels in PD61 CLB $_{\text {w }}$ wile mtDNA expression levels were more than 2-fold higher than control levels. These results also suggest a potential mechanism for the improved mitochondrial morphology, and mitochondrial respiration observed in PD61 $1_{\mathrm{CLB}}$ (Figures 3-2; 3-3A, B; 3-4A, B) and supports our conclusion that mtDNA content in the cells containing CLB in PD61 $1_{\text {Orig }}$ was a driving factor in the functional improvements we observed in PD61 $\mathrm{CLB}$.

Mitochondrial gene expression was unchanged and remained reduced, as compared to control in both PD63 Orig and PD63 ${ }_{\mathrm{CLB}}$ (Figure 3-7A). There was a slight but significant increase (less than 2-fold) in mitochondrial gene copy number in PD63 $3_{\text {CLB }}$ (Figure 3-7B). This increase in ge ne copy number was surprising. We expected a decrease in mitochondrial gene copy number considering the reduced number of cells that scored "high" for nucleoid content (Figure 3-5C, D). Ashley et al. suggested that fusion of nucleoids could occur as cells strive to maintain mtDNA copy number (Ashley et al., 2005). Also, there is a linear relationship between mtDNA content and nucle oid volume (Bereiter-Hahn, 1997). Consequently, the large nuc leoids in PD63 CLB (Figure 3-5C) may harbor increased numbers of mtDNA. Additionally, PD63 ${ }_{\mathrm{CLB}}$ had a decline in mitochondrial function and decrease in mitochondrial respiration, as compared with PD63 Orig (Figures 3-2; 3-3C, D; 3-4C, D). We speculate that this slight increase in mitochondrial gene copy number could be a compensatory mechanism. Since there was 
no change in
mitochondrial ge ne
expression, the
increase in mtDNA
copy number did

not have

downstream

functional

consequences. The

poor cellular and

mitochondrial

function in

PD63 ${ }_{\text {CLB }}$ reflects

this outcome.

As expected, there

was no change in

mitochondrial gene

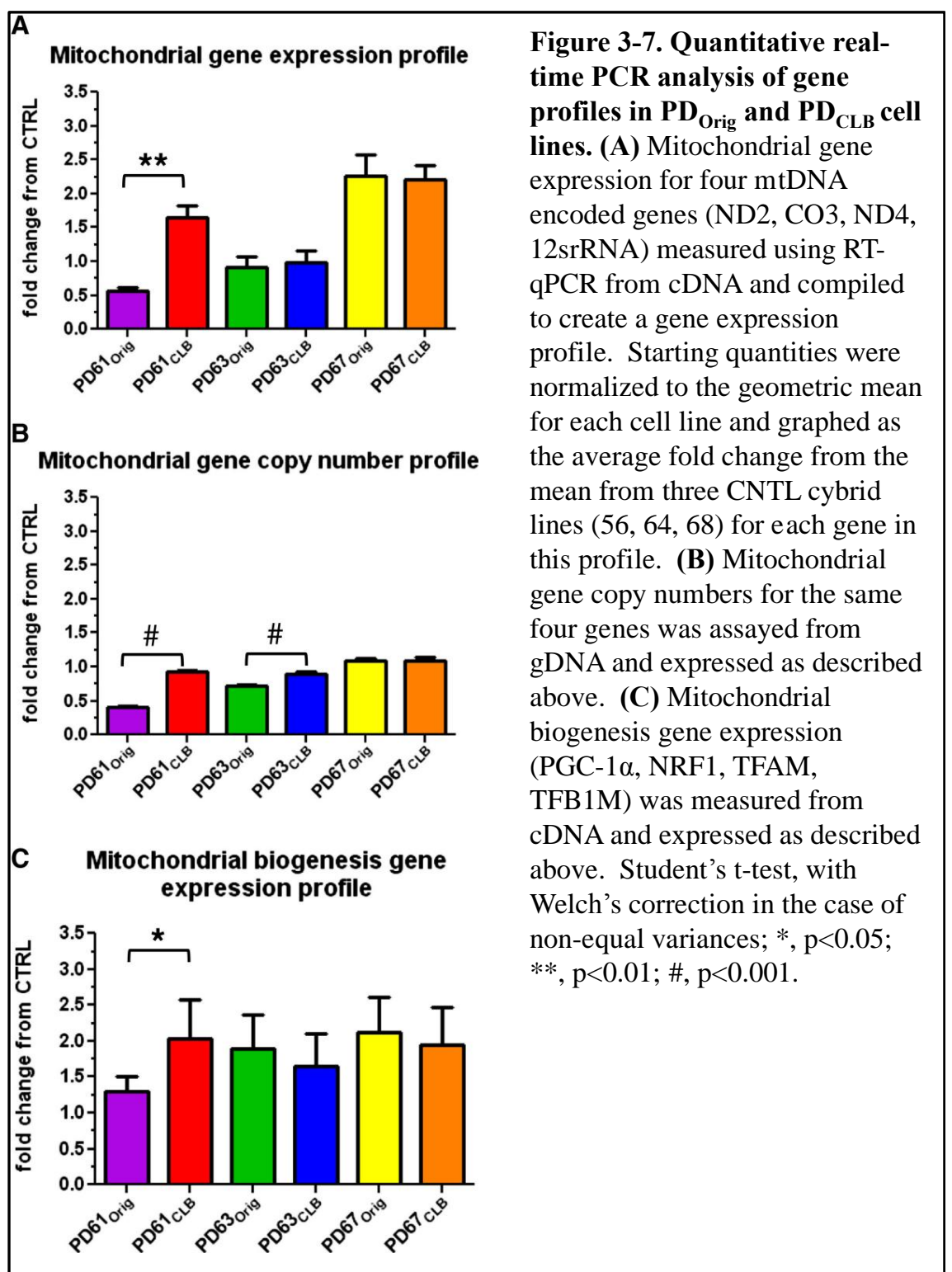

Figure 3-7. Quantitative realtime $P C R$ analysis of gene profiles in $\mathbf{P D}_{\text {Orig }}$ and $\mathbf{P D}_{\mathrm{CLB}}$ cell lines. (A) Mitochondrial gene expression for four mtDNA encoded genes (ND2, CO3, ND4, 12srRNA) measured using RTqPCR from cDNA and compiled to create a gene expression profile. Starting quantities were for each cell iine and graphed as mean from three CNTL cybrid lines $(56,64,68)$ for each gene in this profile. (B) Mitochondrial gene copy numbers for the same four genes was assayed from gDNA and expressed as described above. (C) Mitochondrial biogenesis gene expression (PGC-1 $\alpha$, NRF1, TFAM, TFB1M) was measured from 作 Welch's correction in the case of non-equal variances; *, $\mathrm{p}<0.05$; $*$, $\mathrm{p}<0.01 ; \#, \mathrm{p}<0.001$.

expression or copy number between PD67 $7_{\text {Orig }}$ and PD67 $7_{\mathrm{CLB}}$ (Figure 3-7A, B). Both of

these cell lines exhibited similar mitochondrial morphology, mitochondrial respiration and nucleoid content (Figures 3-2; 3-3E, F; 3-4E, F). These results suggest that creation of PD67 $7_{\mathrm{CLB}}$ from PD67 $7_{\text {Orig }}$ cells containing CLB did not substantially alter mtDNA genetics or phenotypic expression.

Bioge nes is gene expression in $\mathrm{PD}_{\mathrm{Orig}}$ and $\mathrm{PD}_{\mathrm{CLB}}$ cybrid cell lines 
Cellular regulation of mitochondrial biogenesis is critical for the maintenance of a functional pool of mitochondria in neurons (Wareski et al., 2009). In fact, the mitochondrial biogenesis pathway has emerged as a potential therapeutic target for PD (Wareski et al., 2009; Zheng et al., 2010). Peroxisome proliferator-activated receptor gamma co-activator 1-alpha (PGC-1 $\alpha)$ is a transcriptional co-activator and serves as the master regulator of mitochondrial biogenesis (Wu et al., 1999). A genome-wide analys is of PD patients and controls found that PGC-1 $1 \alpha$ expression was reduced in PD patients (Zheng et al., 2010). Overexpression of PGC-1 $\alpha$ in neurons was found to be protective in a neurotoxin mouse model of PD (Mudo et al., 2012). In cell culture, primary fibroblasts and cybrids generated using mtDNA from patients with mitochondrial diseases showed improved respiration after increased PGC-1 $\alpha$ expression (Srivastava et al., 2009). PPAR (peroxis ome proliferator-activated receptor) agonists, such as bezafibrate (an agonist of PPAR $\alpha$ ), have also been shown to improve mitochondrial function in patient fibroblasts and myoblasts (Bastin et al., 2008) and in a mouse model of mitochondrial disease (Wenz et al., 2008).

To explore what role mitochondrial biogenesis plays in the mitochondrial changes found between the $\mathrm{PD}_{\text {Orig }}$ and $\mathrm{PD}_{\mathrm{CLB}}$ lines, we measured the expression of four mitochondrial biogenesis genes including PGC-1 $\alpha$, nuc lear respiratory factor 1 (NRF1), mitochondrial transcription factor B1 (TFB1M) and mitochondrial transcription factor A (TFAM), and used their expression levels to create a mitochondrial gene biogenesis profile. NRF1 is a DNA-binding prote in that serves to positively regulate nuclearencoded subunits of the mitochondrialETC (Scarpulla, 2008). In contrast, TFB1M and TFAM bind directly to mtDNA to initiate mitochondrial-encoded gene transcription. 
(Diaz and Moraes, 2008; Gaspari et al., 2004). These four genes represent control of nuclear- and mitochondrial-encoded mitochondria ETC gene transcription; thereby creating a gene expression profile that can be used to quantitate cell-wide activation of mitochondrial biogenesis.

In PD61 ${ }_{\mathrm{CLB}}$, expression of mitochondrial biogenesis genes was significantly increased by approximately 1.5 -fold compared to PD61 Orig $($ Figure 3-7C). Improved biogenesis is consistent with the increased mitochondrial gene expression and mtDNA copy number described above (Figure 3-7A, B) and with the general improvement in cellular and mitochondrial function in PD61 ${ }_{\mathrm{CLB}}$ compared to PD61 ${ }_{\text {Orig. }}$. This improvement in biogenesis may represent a shift in the population of cybrid cells in PD61 ${ }_{\mathrm{CLB}}$ to include more cells with improved mtDNA gene expression and copy number, or it may represent the improved expression of mitochondrial genes within cells. PGC-1 $\alpha$ enhances mitochondrial ETC function and biogenes is by integrating cellular signals such as AMP/ATP ratios via the AMP-activated kinase (reviewed in Wenz, 2011). Further research on the benefits of increased PGC-1 $\alpha$ signaling would be beneficial for understanding the therapeutic potential of PGC-1 $\alpha$ manipulation.

There was no difference in biogenesis gene expression between PD63 Orig and PD63 $_{\text {CLB }}$ (Figure 3-7C). This was not unexpected due to the decline in mitochondrial function in PD63 $\mathrm{CLB}_{\text {, }}$ compared to PD63 Orig (Figure 3-4C, D). There was also no change in biogenesis gene expression between PD67 $7_{\text {Orig }}$ and PD67 ${ }_{\text {CLB }}$ (Figure 3-7C). This was also expected because there was no difference in mitochondrial gene expression, gene copy number, or mitochondrial function between these two cell lines. 
It is important to remember that in the cybrid model, mtDNA from individual patients is expressed against a common background of nuclear genes from the SH-SY5Y parent cell line. The differences we observed in expression of nuclear mitochondrial biogenesis genes across different cybrid lines are ultimately a consequence of the presence of individual patients' mtDNA. However, the differences in mitochondrial biogenesis signaling across $\mathrm{PD}_{\mathrm{CLB}}$ compared to $\mathrm{PD}_{\text {Orig }}$ cell lines within each cybrid line are derived from selection of CLB-producing cybrids. We found that selection of cybrid cells for CLB expression had a differential effect on mitochondrial biogenesis.

Mitochondrial biogenes is gene expression improved in PD61 ${ }_{\mathrm{CLB}}$, but was unchanged in PD63 ${ }_{\mathrm{CLB}}$ and in PD67 $\mathrm{CLB}$. The mechanism behind the different responses to CLB cloning is unc lear; however our findings suggest that changes in mitochondrial function, gene expression, and biogenesis signaling is driven by the changes in mtDNA we observed, especially in PD61 $1_{\mathrm{CLB}}$.

\section{Discussion}

For this study, we investigated if cloning cells on the basis of CLB expression would lead to a decline in mitochondrial and cellular function. We chose three PD cybrid cell lines based on their heteroge neous mitochondrial function. Surprisingly, we did not see a uniform response in the CLB-cloned cell lines compared to the original cell lines. PD61 ${ }_{\text {CLB }}$ had improved mitochondrial function compared to PD61 Orig versus PD63 ${ }_{\text {CLB }}$, which showed a decrease in mitochondrial function compared to PD63 Orig. There was no difference in mitochondrial function between PD67 ${ }_{C L B}$ and PD67 $7_{\text {Orig. }}$ In an effort to understand the mechanis $m$ behind the heterogeneous responses of each cell line to the CLB cloning, we investigated if there was a difference in mtDNA between each cell line. 
We found that the mtDNA quantity in the cells specifically containing a CLB (i.e. the cells that were cloned to make the new cell lines) was the predictor of mitochondrial function in the clone cell line, not presence or absence of CLB. These studies suggest that mtDNA quantity may play an important role in PD pathogenesis.

One of the most surprising findings in this study was that that there was no

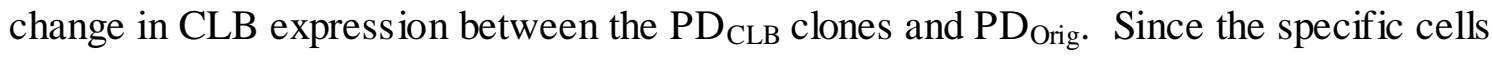
we cloned were selected based on the presence of CLB, we expected to see an increase in CLB expression in the $\mathrm{PD}_{\mathrm{CLB}}$ cell lines. Instead, we saw no difference in CLB size or frequency in the $\mathrm{PD}_{\mathrm{CLB}}$ cell lines compared to $\mathrm{PD}_{\text {Orig. }}$. In PD substantia nigra, the expression level of LB appears to be constant (3-4\%) irrespective of the duration of the disease. This observation is cons istent with the idea that LB are constantly forming and being eliminated (Lees et al., 2009). The frequency of CLB expression in PD $_{\mathrm{CLB}}$ clones and parent $\mathrm{PD}_{\text {Orig }}$ cybrid cell lines was also comparable to the frequency of LB found in PD patient brain (Greffard et al., 2010).

To understand why increased CLB expression was not sustained in PD $_{\text {CLB }}$ cybrid lines, it is important to think of CLB as aggresomes (Olanow et al., 2004). Aggresomes can be created in vitro by transiently inducing protein misfolding or the overexpression of prote ins that are prone to misfolding (Olanow et al., 2004). Because the generation of misfolded and damaged proteins is continuous in PD cybrid lines, we consider CLB to be “professional aggresomes.” Like aggresomes, CLB form in the perinuclear region, contain punctate gamma tubulin staining and are composed of aggregated, damage d and misfolded prote ins (Trimmer et al., 2004). Some have speculated that LB are permanent trash dumps, while others consider LB to be recycling centers (Olanow et al., 2004). One 
way to determine if CLB are trash dumps or recycling centers is to visualize the changes in the distribution of fluorescently labeled prote ins in CLB in live cells. Some of these experiments will be described in a later chapter.

There are several potential mechanisms that could contribute to the level of CLB expression in PD cybrid lines. Studies have shown that aggresomes are asymmetrically distributed during somatic and stem cell mitosis (Rujano et al., 2006). One daughter cell retains the aggresome while the other is free of damaged and misfolded proteins (Fuentealba et al., 2008; Rujano et al., 2006). The same asymmetric inheritance of aggresomes also happens when a cybrid cell with a CLB undergoes mitos is. If CLB-free daughter cybrid cells proliferate more efficiently than cells with the burden of a CLB, the frequency of CLB-positive cells would decline during cell line expansion after initial subcloning. CLB-positive cells are not completely eliminated from cybrid lines because prote in misfolding and aggregation is an ongoing process. Each $\mathrm{PD}_{\text {Orig }}$ and $\mathrm{PD}_{\mathrm{CLB}}$ cybrid line achieved a steady state level of CLB expression comparable to what has been previously described in vivo and in vitro (Rujano et al., 2006; Trimmer et al., 2004).

Another potential mechanism that may play a role in the steady state level of CLB expression is cytoplasmic extrusion. Extracellular LB have also been identified in PD brain sections using aSYN antibodies (Alafuzoff et al., 2009). Doehner. et al characterized the accumulation of granular Reelin/ $\square$-amyloid deposits in mouse hippocampus (Doehner et al., 2012). They detected Reelin-positive "budding-like" extrusions that they claim represent a protective reaction by postmitotic neurons with impaired protein degradation pathways. The extruded misfolded proteins, mitochondria, vacuoles and debris are then cleared by intrinsic glia (Doehner et al., 2012). Extracellular 
CLB have been seen in cultures of PD cybrid cell lines that may be the result of cytoplas mic extrusion. The time-lapse studies of CLB-expressing cells could be used to conf irm this possibility.

The clinical significance of LB has been widely debated (Jellinger, 2009). LB expression in the basolateral nucleus has been associated with visual hallucinations in PD (Harding et al., 2002a). Also, there are increased numbers of LB in demented versus non-demented PD brain sections from cortex, limbic structures and amygdala (Harding et al., 2002b). Several research groups have argued that LB are detrimental and contribute to neuronal degeneration in PD (Galvin et al., 1999; Lu et al., 2005; Shults, 2006). Harrower et al., in contrast, proposed that LB mark a "struggling cell," a concept of the LB that is consistent with the results presented in this paper (Harrower et al., 2005). Several groups have suggested that LB are formed by neurons in a effort to maintain normal function in the face of an ongoing pathology (Au and Calne, 2005; de la FuenteFernandez et al., 1998; Harrower and Barker, 2005; Hindle, 2010; Kramer and SchulzSchaeffer, 2007; Wakabayashi et al., 2007). The nature of the pathological process that results in LB formation has also been a subject of speculation. Other groups have suggested that mitochondrial dysfunction precedes and drives LB pathology and neurological dysfunction in PD (Lin et al., 2012; Zhou et al., 2011). The results presented in this chapter support the proposal that mitochondrial dysfunction is a major contributor in the development of CLB pathology. It is important to note that models of mitochondrial dysfunction, using MPTP or rotenone, do not form LB (Banerjee et al., 2009), suggesting that CI inhibition alone is not sufficient to drive CLB formation. 
If CLB are detrimental for cells, then all three $\mathrm{PD}_{\mathrm{CLB}}$ lines should have exhibited worsening of cellular and mitochondrial functions. This did occur in PD63 ${ }_{\mathrm{CLB}}$, but not in PD61 $1_{\mathrm{CLB}}$ or PD67 $7_{\mathrm{CLB}}$. If CLB are an asset with beneficial functions, then cellular and mitochondrial function should improve. This was the outcome in PD61 $1_{\mathrm{CLB}}$ but not in PD63 $3_{\mathrm{CLB}}$ or PD67 $\mathrm{CLB}$. Sub-cloning CLB-expressing cells to produce PD67 $7_{\mathrm{CLB}}$ did not substantially alter cellular or mitochondrial function. Our data suggest that CLB expression does not drive the dysfunction in $\mathrm{PD}_{\text {Orig }}$ or $\mathrm{PD}_{\mathrm{CLB}}$ cybrid lines.

Our studies of $\mathrm{PD}_{\text {Orig }}$ and $\mathrm{PD}_{\mathrm{CLB}}$ cybrid cell lines also suggest that the mtDNA is key to the expression of cellular and mitochondrial dysfunction, such as altered ETC activity and oxygen utilization, abnormal mitochondrial morphology, etc. The $\mathrm{PD}_{\mathrm{CLB}}$ cell lines that featured significant changes in mitochondrial function and morphology also had distinct changes in mtDNA copy number and/or expression from the $\mathrm{PD}_{\text {Orig }}$ lines. Our data supports the proposition by Esteves et al. that mtDNA is at least partly responsible for mitochondrial ETC defects in sporadic PD (Esteves et al., 2010). Exner et al. further concluded that mitochondrial dysfunction is a "common denominator" in the pathogenes is of sporadic and familial PD (Exner et al., 2012).

One intriguing finding in our data was the identification of individual cells with CLB that appeared to lack nucleoids and functional mtDNA (see Figure 3-6B). Since formation of an aggresome or CLB is ATP-driven, it seems unlikely that a cell without mtDNA could generate a CLB. This idea leads to speculation that loss of mtDNA and nucleoids could be part of PD pathogenes is. CLB-bearing PD cybrid cells without mtDNA or nuc leoids can survive in culture because of the supportive culture conditions. Nucleoid-free and mtDNA-free neurons are unlike ly to survive in vivo unless they can 
derive sufficient support from surrounding glia. Future studies will be designed to confirm these findings, by investigating nuc leoid content in post-mortem tissue, specifically comparing neurons with or without LB.

The purpose of the studies in this chapter was to investigate how increasing CLB expression in three PD cybrid cell lines by sub-cloning based on the presence of Congo red staining would alter cellular and mitochondrial function. Although we expected to find a consistent decline in the cellular and mitochondrial function of the cloned cells compared to the original cell lines, we in fact found a range of changes. The individual cells in the $\mathrm{PD}_{\text {Orig }}$ cell lines that contained CLB were diverse in their mtDNA content, as shown by presence or absence of nucleoids. In the end, it was this mtDNA content that predicted the phenotypic outcomes, such as mitochondrial respiratory function and morphology. If the CLB-containing cells contained nucleoids, than the resulting clones would have improved or unchanged mitochondrial function. In the case of the CLBcontaining cells without nuc leoids, the resulting clone had a decline in mitochondrial function. These data further indicate that there is a complex relationship between the formation of CLB and mitochondrial dysfunction. Since presence or absence of nucleoids was not a consistent predictor of CLB expression, we cannot conclude that mtDNA content exclusively drives CLB formation. It is still like ly that mitochondrial dysfunction is an important contributor to the formation of CLB. The studies described in the next chapters further investigate the complex relationship between mitochondrial function and CLB formation. 


\section{Expression of the yeast NADH dehydrogenase improves mitochondrial function in a cell model of Parkinson's disease}

\section{Introduction}

CI, also called NADH dehydrogenase, is dually encoded by the mtDNA and gDNA. CI has 7 subunits encoded by the mtDNA in addition to the 38 subunits encoded by the gDNA (Papa et al., 2012). Functionally, CI accepts electrons from NADH at the origin of the ETC and passes the electrons on to CIII via coenzyme Q (Yagi and Matsuno-Yagi, 2003). CI is also an important site of free radical production, especially in the brain (Barja and Herrero, 1998). Dysfunction of complex I has been observed in the brain as well as in the peripheral tissues of sporadic and familial PD patients (Arthur et al., 2009; Haas et al., 1995; Schapira et al., 1990a; Swerdlow et al., 1996; Winklhofer and Haass, 2010). Furthermore, neurotoxins, such as rotenone and MPTP, which selectively inhibit complex I, have been found to induce parkinsonis m (Cannon and Greenamyre, 2010; Richardson et al., 2007; Sherer et al., 2007). CI in PD brain is also oxidatively damaged and could contribute to PD pathogenes is by further increasing free radical production and oxidatively damaging mtDNA and mitochondrial proteins (Keeney et al., 2006). Oxidative damage to proteins, specifically aSYN, has been implicated to play a role in prote in aggregation and LB formation (Beyer, 2006; Ischiropoulos, 2003).

Several potential therapies for diseases with CI dysfunction, including PD, have targeted mitochondria. For example, creatine is naturally occurring compound that has been shown to play an important role in energy production in muscles and nerve cells. Clinical trials using creatine to treat PD patients have not been futile (Beal, 2011; 
Chaturvedi and Beal, 2012) and a full-scale clinical trial to study creatine treatment over 5-7 years is on going. MitoQ is a mitochondrial-targeted antioxidant that has also been studied as a potential mitochondrial therapy in various animal models of mitochondrial dysfunction; however, a study of MitoQ as a neuroprotection agent in PD was unsuccessful (Smith and Murphy, 2010). Currently, studies in PD patients have not shown that these and other neuroprotective compounds can consistently and effectively improve PD symptoms or disease progression (Chaturvedi and Beal, 2012).

An alternate approach that has been pursued in a variety of PD models is to bypass or supplement $\mathrm{CI}$ in order to reduce mitochondrial ETC dysfunction. The yeast strain Saccharomyces cerevisiae contains both internal and external single subunit NADH dehydrogenase enzymes. The internal NADH dehydrogenase (Ndi1) faces the mitochondrial matrix where NADH is formed via the Krebs cycle (Marres et al., 1991). Similar to mamma lian CI, Ndi1 accepts electrons from NADH and passes them on to coenzyme Q. However unlike mammalian CI, Ndi1 is a single, monogenic protein encoded by the NDII gene and is insensitive to the complex I inhibitor rotenone (de Vries and Grivell, 1988). Studies have found that this yeast prote in can be expressed in mammalian cells and, notably, Ndil expression does not have a negative effect on cell growth or viability in human cell lines, such as HEK293, 143B, and PC12 cells (Marella et al., 2007; Seo et al., 1999; Seo et al., 2000).

In light of the observed CI impa irment in PD and other diseases, Ndil has been studied as a potential therapy. It has been reported that expression of Ndil improved oxidative phosphorylation capacity in CI defic ient 143B cells (Bai et al., 2001) and in a cell model of Leber's hereditary optic neuropathy (LHON) containing a complex I 
mutation (Park et al., 2007). The effects of Ndil have also been investigated in animal models. One study found that Ndil could be expressed, was localized to mitochondria where it was functionally active, and did not result in an inflammatory response in rodents (Seo et al., 2004). Importantly, in animal models of PD, it was reported that Ndil expression improved cell survival after neurotoxin insult (Barber-Singh et al., 2010; Barber-Singh et al., 2009). One major drawback is that these experiments used 1methyl-4-phenyl-1,2,3,6-tetrahydropyridine (MPTP) or rotenone to create PD animal models. The effect of the toxic derivative of MPTP (MPP+) on Ndil has not been demonstrated but it is well established that Ndil is resistant to rotenone (de Vries and Grivell, 1988). In light of these studies, it is important to investigate if expression of Ndil would affect mitochondrial function in a non-toxin, human cell model of spora dic PD.

As our findings described in Chapter 1 showed selecting cells for CLB expression did not result in a consistent change in mitochondrial function. Mitochondrial function was driven by the mtDNA in the cells, independent of the presence of absence of CLB expression. In light of these results, our next line of investigation was to bypass the defective CI in a PD cybrid cell line with Ndil to determine if this would alter CLB expression or formation. The significance of CI to PD suggests that expression of Ndil to bypass $\mathrm{CI}$ may be a valid therapeutic approach for reducing the mitochondrial dysfunction observed in PD patients.

In order to examine the therapeutic effect of Ndil transfection, we investigated if expression of Ndi1 improves mitochondrial function in both normal SH-SY5Y cells and a PD cybrid cell line that exhibited reduced levels of mitochondrial function. We also 
evaluated if Ndi1 expression changes endogenous ETC prote in transcription and assembly. Lastly, we explored if improving mitochondrial function with Ndi1 alters prote in aggregation, another pathological hallmark of PD. Our results indicate that Ndil expression does improve mitochondrial respiratory function, without adversely affecting endogenous ETC assembly, and reduces protein aggregation but not CLB expression. These data suggest that Ndil expression has potential as therapy to improve mitochondrial function in sporadic PD.

\section{Methods}

\section{Immunocytochemistry, Congo red staining, Cellular respiration, and qRT-PCR}

Methods performed as described in General Methods (Chapter 2).

\section{Ndi1 transfection}

Cells were transfected with the rAAV-NDII (serotype 2), as previously described (Seo et al., 2000). Briefly, PD61 and SY5Y cells were plated for 48 hours in 6-well plates before addition of the virus. Viral transfection used $5-8 \times 10^{12}$-virus partic les $/ \mathrm{mL}$ to ensure efficient transfection (Seo et al., 2002). Cells were incubated with virus containing medium for 5 days, then returned to regular GM and allowed to proliferate. Transfected cell populations were then grown in selection media ( $5 \mathrm{mM}$ galactose $/ 30 \mathrm{nM}$ rotenone) for at least 2 weeks. Selection media was made with glucose-free DMEM (Life Technologies), supplemented with 10\% FBS, pyruvate/uridine, antibiotic/antimycotic, $5 \mathrm{mM}$ galactose and $30 \mathrm{nM}$ rotenone. Rotenone was prepared in advance as a stock and added directly to the medium during the selection period. Ndil transfected cell lines were maintained in GM but were returned to selection media every 5 days for 48 hours to 
mainta in selection for Ndi1 expression. Immunocytochemistry with an Ndi1 specific antibody was used to verify that the selection process was complete (Barber-Singh et al., 2010). Ndil expressing cell lines were removed from selection media briefly before preparation of frozen stocks for later use.

\section{Results}

\section{Ndi1 expression in SH-SY5Y cells and a PD cybrid cell line}

Successful transfection and selection of PD61 and SY5Y cell lines for expression of rAAV-NDI1 (PD61 ${ }^{\mathrm{NDI} 1}$ and SY5Y ${ }^{\mathrm{NDI1}}$ ) was determined using immunocytoche mistry, qRT-PCR, and immunoblotting. Incorporation of Ndil prote in into mitochondria was confirmed based on the co-localization of Ndil immunostaining with immunostaining for CV-alpha (Figure 4-1A, B and inserts) and by detection of Ndil in PD61 ${ }^{\mathrm{NDI1}}$ and SY5Y ${ }^{\mathrm{NDI1}}$ by Western blot. Ndil protein expression levels were 3-fold higher in PD61 $^{\text {NDI1 }}$ than in SY5Y ${ }^{\text {NDI1 }}$ (Figure 4-1C). Similarly, using qRT-PCR, NDI1 gene expression was 6-fold higher in PD61 ${ }^{\mathrm{NDI1}}$ than in SY5Y ${ }^{\mathrm{NDI1}}$ (Figure 4-1D). To confirm the specificity of our primers and antibodies, we measured Ndil protein and gene expression levels in the non-transfected SY5Y and PD61 cell lines. There was no detectable signal for Ndil in either of these cell lines.

\section{Ndi1 expression improves mitochondrial oxygen consumption}

Oxygen utilization in non-transfected and Ndi1 expressing cell lines was assessed using the Seahorse XF24 Analyzer (Gerencser et al., 2009). The basal respiratory function observed in PD61 was relatively low; it had less than 20\% the basal OCR of SY5Y (Figure 4-2A, B). However, expression of Ndi1 in PD61 ${ }^{\mathrm{NDIl}}$ had a robust effect on respiration, resulting in a 10-fold increase in basal OCR levels compared to PD61 (Figure 
4-2C). There was also a significant increase in ATP production and maximum respiratory capacity following expression of Ndi1 (Figure 4-2D, E). The significant increases in mitochondrial respiration following Ndil expression also resulted in increased spare capacity and non-mitochondrial respiration, but did not alter the coupling efficiency of

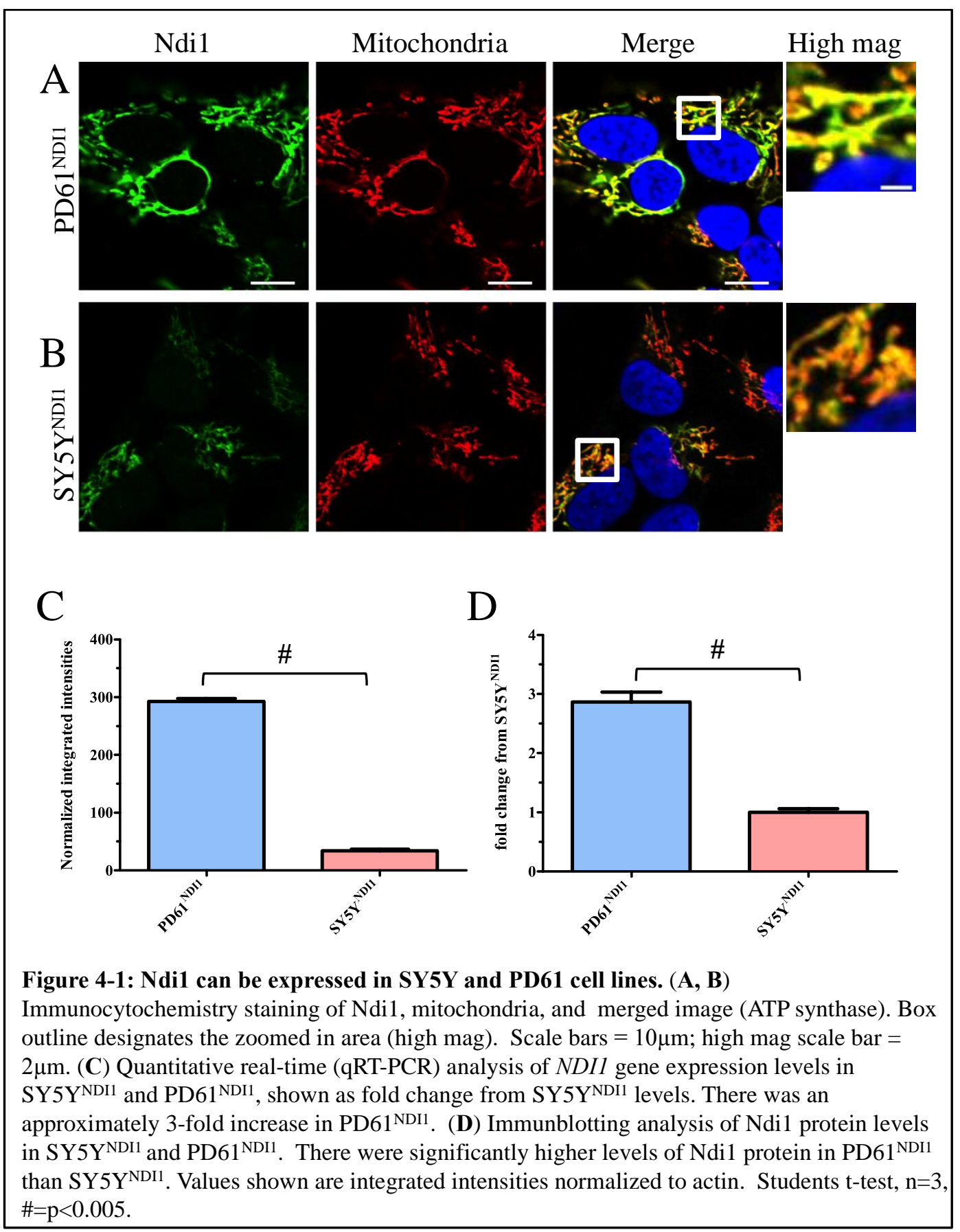


mitochondria in PD61 ${ }^{\mathrm{NDI1}}$.

Ndil expression in SY5Y cells had no effect on basal OCR but did result in a significant decrease in ATP-linked OCR and a significant increase in maximum capacity OCR (Figure 4-2A, C-D, left set of bars labeled STD). There was also a significant increase in spare capacity, as well as in the coupling efficiency, but no change in the nonmitochondrial function.

We measured oxygen utilization in the presence of $30 \mathrm{nM}$ rotenone (rotenone pretreatment or Pre-Rote) to confirm that Ndi1 was functionally contributing to the changes seen in ETC function (Figure 4-2C-H). Under these conditions, rotenone will inhibit any endogenous CI activity but will not affect the activity of rotenone resistant Ndil in expressing cells. Since CI is located at the beginning of the ETC and acts like a pacesetter for ATP generation (see Papa and De Rasmo, 2013), we were not surprised to find that basal, ATP-linked and maximum capacity OCR in non-transfected cells (SY5Y) was significantly reduced by pretreatment with rotenone (Figure 4-2F-H, red bars, PreRote). Pretreatment with rotenone also significantly decreased ATP-linked and maximum capacity OCR in PD61 (Figure 4-2D-E, dark blue bars). By comparing the light blue bars in Figures 2F,H (PD61 ${ }^{\text {Ndi1 }}$, Pre-Rote) it was clear that rotenone pretreatment also resulted in only a slight, but significant, reduction in basal and maximum capacity OCR. There was also a slight decline in maximum capacity OCR in $\mathrm{SY} 5 \mathrm{Y}^{\mathrm{NDI1}}$ after rotenone pretreatment (Figure 4-2H, STD vs. Pre Rote).

Despite pretreatment with $30 \mathrm{nM}$ rotenone to suppress endogenous CI, significant improve ments in basal, ATP-linked and maximum capacity OCR were detectable in PD61 $^{\text {NDI1 }}$ (Figure 4-2F, Pre-Rote). The functional bypass of CI in SY 5Y ${ }^{\text {NDI1 }}$ cells also 


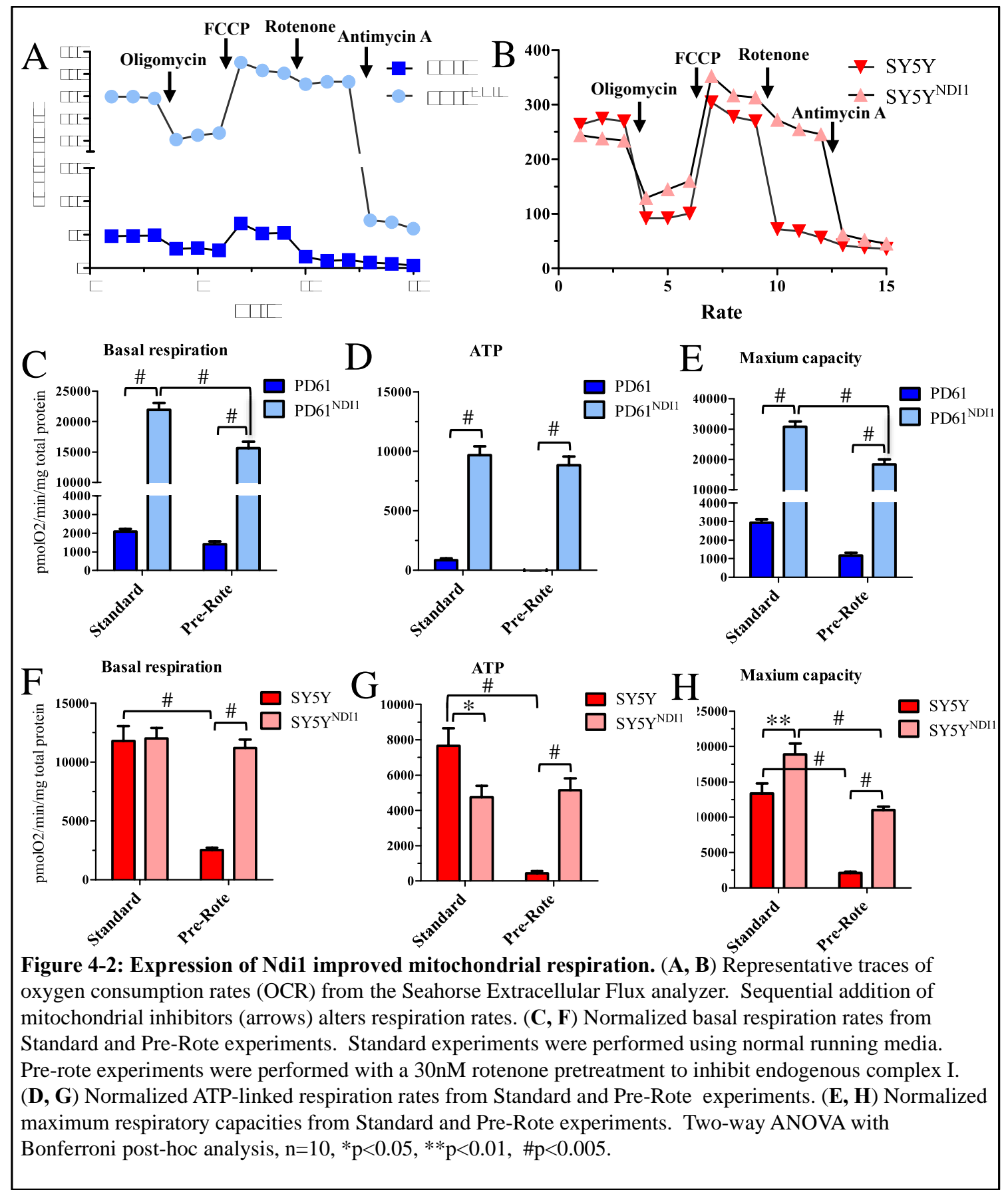

resulted in significantly improved basal, ATP-linked and maximum capacity OCR in

cells pretreated with $30 \mathrm{nM}$ rotenone to inhibit native complex I activity (Figure 4-2C-E

STD vs. rotenone pre-treatment, light red bars).

We observed a 10-fold change in OCR PD61 ${ }^{\mathrm{NDI} 1}$ compared to PD61 and a compensatory $50 \%$ decrease in the extracellular acidification rate (ECAR). This is 
consistent with the reduction in glycolysis that de velops because OCR is increased in response to the presence of Ndil (Dranka et al., 2012). There was also a slight decrease in ECAR in SY5Y ${ }^{\mathrm{NDI1}}$ compared to SY 5Y. This suggests that the cell lines expressing Ndil have shifted mitochondrial oxygen utilization to become more reliant on OXPHOS than glycolysis.

\section{Effect of Ndi1 expression on mitochondrial morphology}

Abnormal mitochondrial morphology is a characteristic feature of PD cybrid lines at both the light microscopy and ultrastructural levels (Cronin-Furman et al., 2013; Trimmer et al., 2000). In light of the improvements in mitochondrial function in

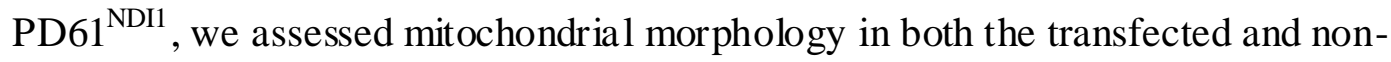
transfected cells lines immunostained for mitochondrial ETC subunit prote in complex V alpha and Ndi1 protein. The frequency of cells containing abnormal, fragmented mitochondria in PD61 ${ }^{\mathrm{NDI1}}$ declined by $8.7 \%$ compared to PD61. Mitochondrial morphology in SY5Y ${ }^{\mathrm{NDI1}}$ was unaffected compared to SY 5Y.

\section{Ndi1 expression increase d mitochondrial gene expression}

We measured the gene expression and copy number of two CI subunits, ND2 and ND4, as well as the CIV subunit COX3, and the 12s ribos omal RNA (rRNA) genes that are all encoded by mtDNA. The copy number of all four genes in PD61 was significantly below the SY5Y level (Figure 4-3C). Mitochondrial gene expression was significantly increased in PD61 ${ }^{\mathrm{NDI1}}$ compared to PD61 for ND2, ND4, and COX3 (Figure 4-3B). In fact, the bypass of CI dysfunction in PD61 ${ }^{\mathrm{NDIl}}$ also resulted in mtDNA gene copy numbers that were significantly increased to levels above SY5Y gene expression levels (Figure 4-3D). Gene expression for ND2, ND4, Cox3 and 12s rRNA was also increased 
by Ndi1 expression in SY5Y ${ }^{\mathrm{NDI1}}$ however changes did not achieve significance (Figure 43B).

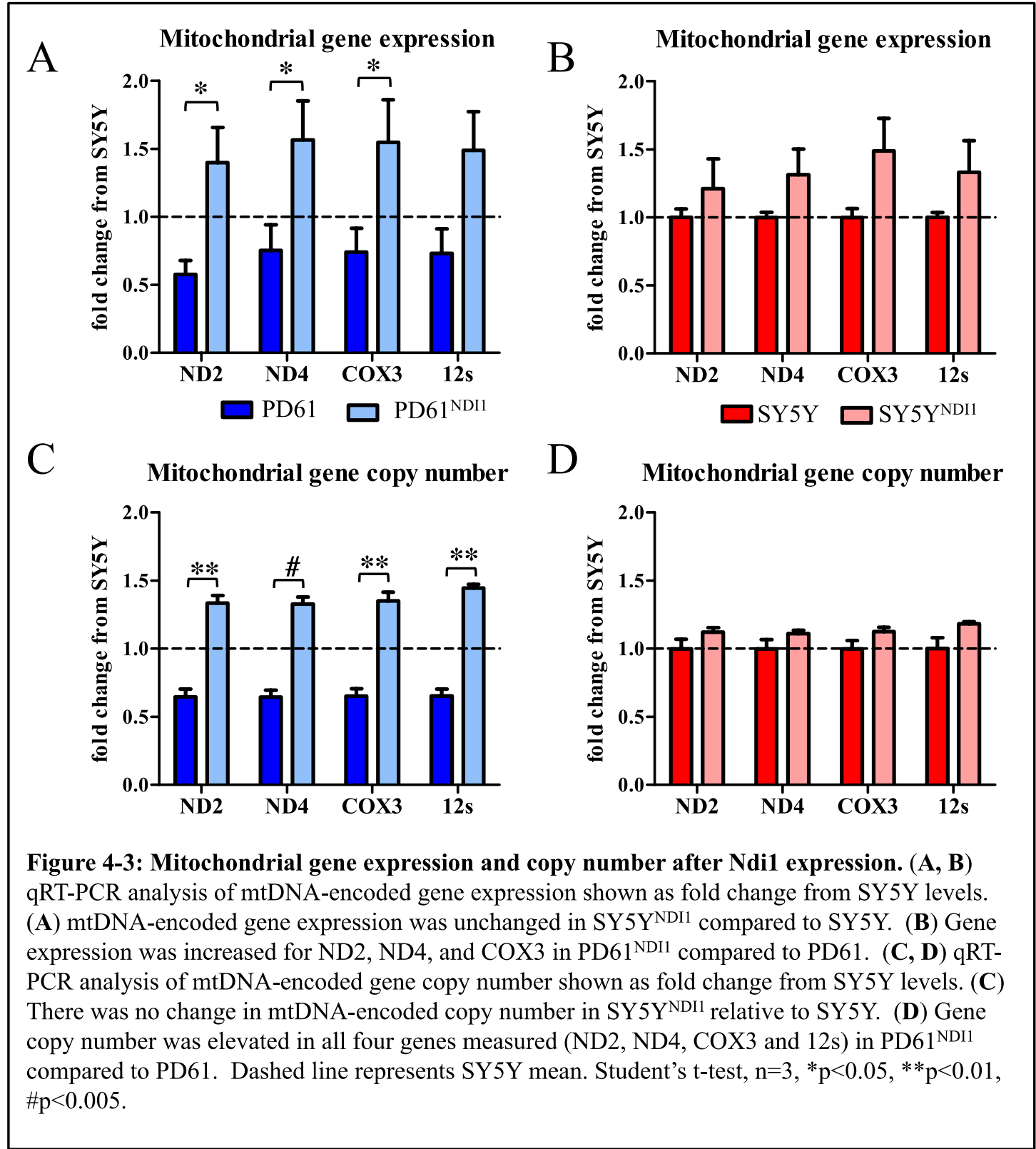

\section{Ndi1 expression incre ase d mitochondrial bioge nes is signaling}

Changes in mitochondrial biogenesis could be responsible for the increases in mitochondrial gene copy number. PCG- $1 \alpha$ is the transcription factor that serves as the master regulator for mitochondrial biogenesis (Wu et al., 1999). In both cell lines expressing Ndi1, we observed a significant increase in PGC-1 $\alpha$ express ion compared to 
the non-transfected cell lines (Figure 4-5A). PD61 ${ }^{\mathrm{NDI} 1}$ had the highest relative expression of PGC-1 $\alpha$, which was not surprising given the robust changes in mitochondrial function and mtDNA gene expression.

\section{Expression of Ndi1 increase d ETC complex assembly}

Considering the increases in mitochondrial gene expression and biogenesis signaling, we also measured the assembly of the individual ETC complexes using

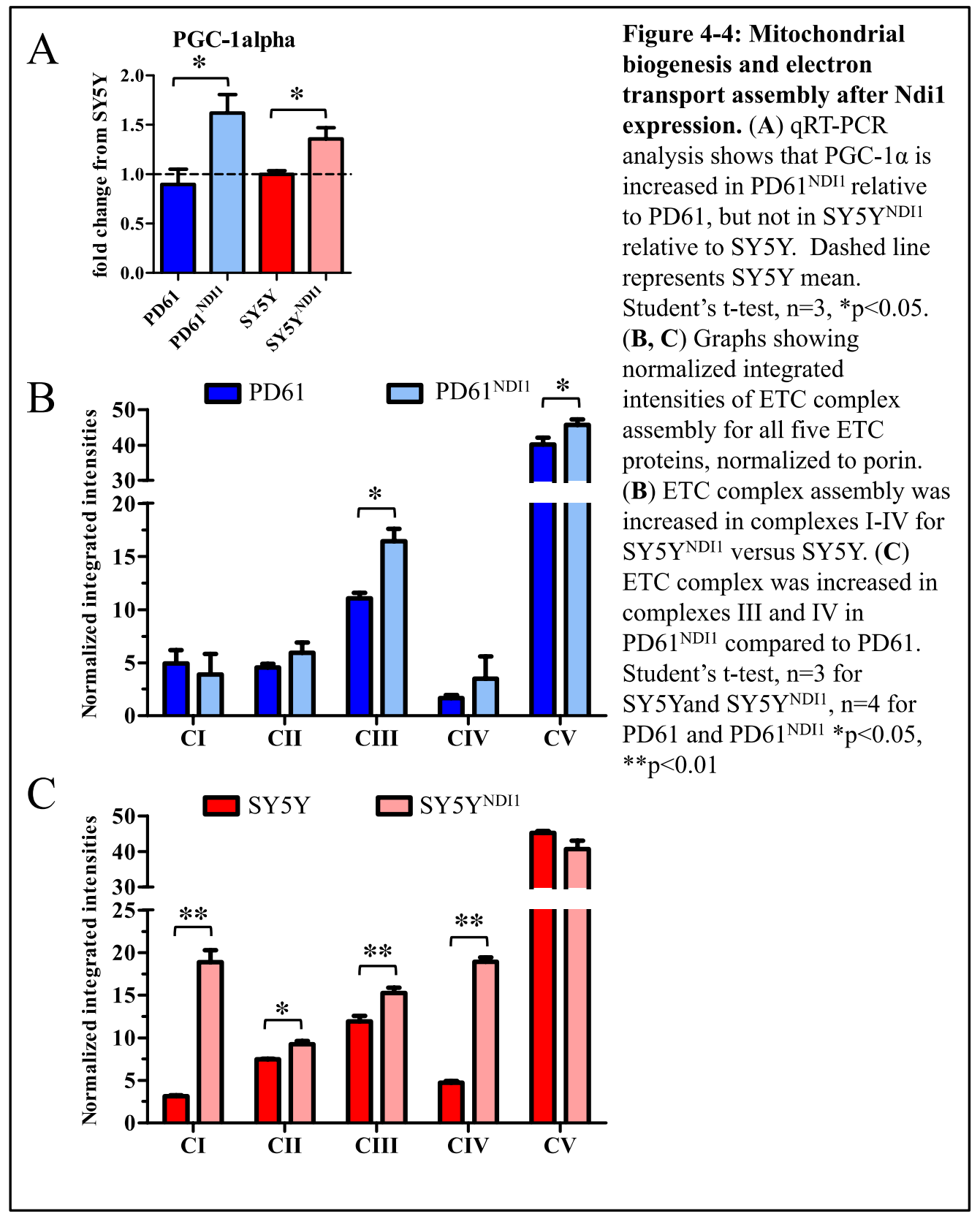


antibodies that recognize correctly assembled ETC complexes (Capaldi et al., 2004). Only CIII and CV displayed significantly increased assembly in PD61 ${ }^{\mathrm{NDI1}}$ (Figure 4-4A). Although there was a significant increase in mtDNA gene expression of subunits for CI and CIV (Figure 4-3A), we did not find any significant changes in assembly of these complexes in PD61 ${ }^{\mathrm{NDI}}$. In contrast, assembly of CI, CII, CIII, and CIV was increased in SY5Y $\mathrm{Y}^{\mathrm{NDI1}}$ compared to SY5Y (Figure 4-4B). In comparis on, mtDNA gene expression of CI and CIV subunits were not significantly changed by Ndi1 expression.

Expression of Ndi1 decreased total cellular aggregated protein content but did not alter CLB expression

We determined the total cellular level of aggregated proteins, as well as the frequency of CLB expression using Congo red (Conway et al., 2000; Cronin-Furman et al., 2013; Khurana et al., 2001). In PD61 ${ }^{\mathrm{NDI1}}$, total cellular aggregated protein content (Congo red positive pixels/cell) was decreased, compared with PD61

(Figure 4-5A). A similar change was observed in SY5Y ${ }^{\text {NDI1 }}$ compared to

SY5Y (Figure 4-5A). CLB continued to be expressed in PD61 ${ }^{\text {Ndi1 }}$

(Figure 4-6B) despite significant improve ment in

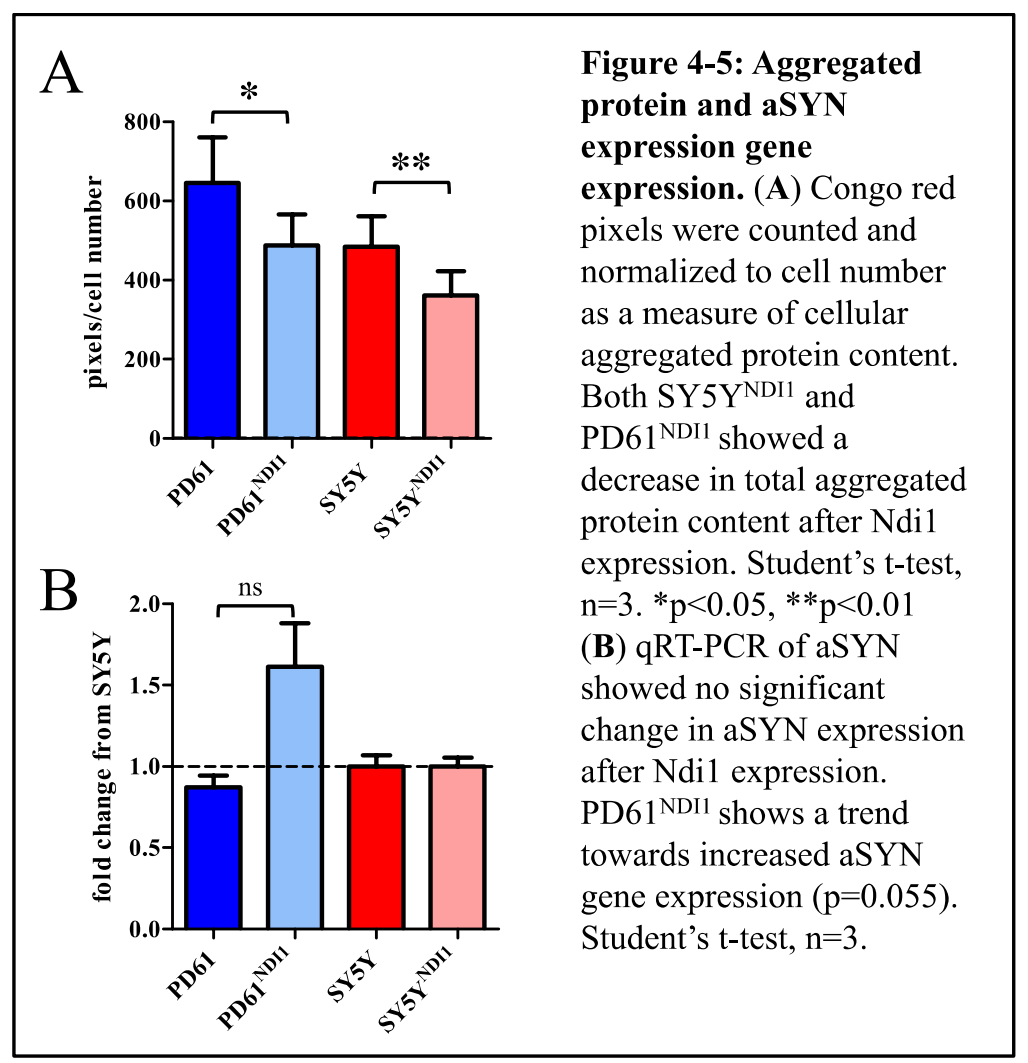


OXPHOS and increased mitochondrial gene expression, mitochondrial biogenesis signaling, and ETC complex assembly. Furthermore, there was no change in frequency or size of CLB in PD61 ${ }^{\text {NDI1 }}$ compared to PD61. Figures 4-6C and D also show that CLB were clearly present in transfected cells with robust Ndil expression. Interestingly, Ndi1 immunostaining was heterogeneously incorporated into CLB (Figure 4-6). In some cases, CLB contained Ndi1 immunostaining that colocalized with staining for porin, the mitochondrial outer membrane prote in (Figure 4-6D).

Finally, we measured aSYN gene expression as aSYN is a major component of LB (Spillantini et al., 1997). Expression of aSYN mRNA was quantified using qRTPCR. As shown in Figure 6B, aSYN gene expression was increased two-fold in PD61 ${ }^{\text {NDI1 }}$ versus PD61 but the change did not achieve statistical significance $(\mathrm{p}=0.055)$. There was also no significant difference between SY5Y ${ }^{\mathrm{NDI1}}$ and SY5Y (Figure 4-5B).

\section{Discussion}

The experiments detailed in this study were designed to determine if bypass of CI dysfunction by the yeast mitochondrial NADH dehydrogenase Ndi1 in a human cell culture model of sporadic PD could improve mitochondrial function and reduce CLB expression. Mitochondrial dysfunction and prote in aggregation are both pathological hallmarks of PD (Banerjee et al., 2009; Dauer and Przedborski, 2003). In the cybrid model of PD, Ndil gene expression significantly improved mitochondrial respiration in both a PD cybrid line, PD61 and the host SY5Y cell line. Furthermore, endogenous mitochondrial ge ne expression and mitochondrial biogenesis signaling via PGC-1 $\alpha$ was increased. In light of the improved mitochondrial function in PD61 ${ }^{\mathrm{NDI} 1}$, we measured total cellular aggregated protein levels. While levels of aggre gated proteins were reduced 


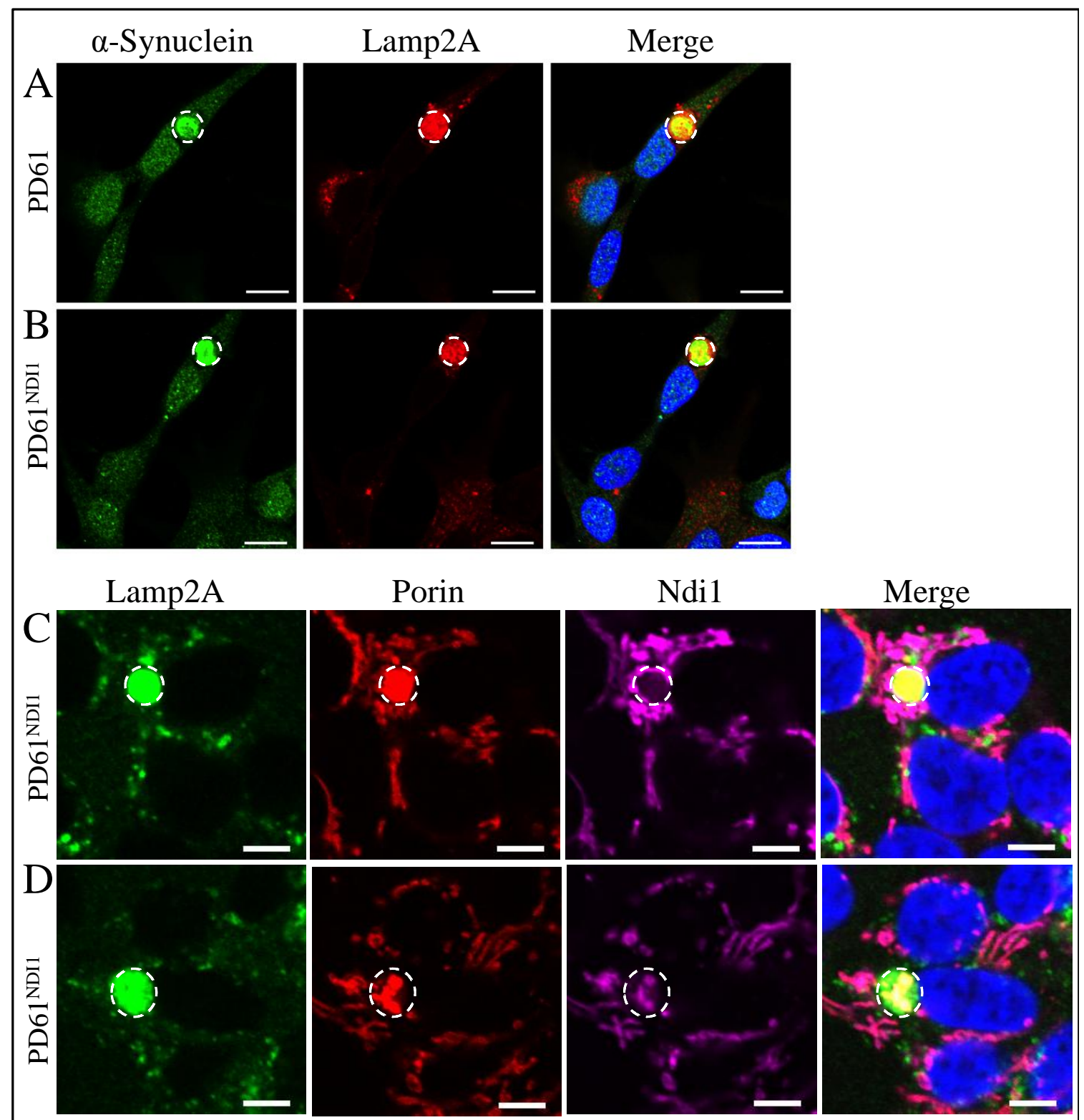

Figure 4-6: Cybrid Lewy body expression after NDI1 expression. (A, B) Representative CLB from PD61 and PD61 ${ }^{\text {NDI1 }}$ showing colocalization of aSYN with Lamp2A. Scale bars $=10 \mu \mathrm{m}(\mathbf{C}, \mathbf{D})$ Representative images from PD61 ${ }^{\mathrm{NDI} 1}$ showing CLB present in cells expressing Ndi1. Scale bars $=5 \mu \mathrm{m}$.

in cells expressing Ndi1, CLB expression was unchanged. This suggests using Ndil to

bypass $\mathrm{CI}$ in a model of sporadic PD by Ndi1 expression improved mitochondrial dysfunction, one of the main pathological features of PD but failed to reduce the formation and expression of CLB.

PD61 has compromised respiratory function relative to SY5Y cells. After expression of Ndi1, we observed significant increases in mitochondrial respiration rates. 
Although we expected some functional improvements, we were surprised by the magnitude of the respiratory improve ments found in this cell line. One of the interpretations of this data is that there is a significant increase in the volume of electrons being transferred along the ETC, leading to the increased oxygen consumption we observed using the Seahorse XF. This could be due to in additive effect of Ndi1 and endogenous CI both accepting electrons; however the respiration experiments run in after pre-treatment with rotenone suggest that very little oxygen consumption was dependent on endogenous-CI compared to Ndil in PD61 ${ }^{\mathrm{NDI1}}$. We did observe a significantly higher rate of non-mitochondrial respiration, i.e. respiration after the antimycin A injection. This could be due to increases in the oxygen consumption activity of other nonmitochondrial pathways that use oxidases (see Brand and Nicholls, 2011). It is important to note that we did not change the coupling efficiency of PD61 ${ }^{\text {NDI1 }}$ compared to PD61. The coupling efficiency is the amount of oxygen consumption that is linked to ATP production. Although previous studies have suggested that coupling efficiencies are very sensitive to changes in proton leak and ATP demand, it has also been suggested that if proton leak increases simultaneous with an increase in respiration rates, there is no change in the coupling ratios (Brand et al., 1993). Ndil does not pump protons; however, the amount of respiration after oligomycin treatment in PD61 ${ }^{\mathrm{NDI1}}$ was significantly higher than PD61 (Figure 4-2A, see post oligomycin respiration) suggesting an increase in proton leak. This increase in leak coupled with the significant increases in overall OXPHOS could explain why we saw no change in the coupling efficiency between these two cell lines. 
In light of the observed functional changes after expression of Ndil, we also measured mitochondrial gene expression. Reduced expression of mitochondrial genes in PD has been observed in previous studies on human brain, peripheral cells, and cybrids (Borland et al., 2009; Cronin-Furman et al., 2013; Keeney et al., 2009; Zheng et al., 2010). We found increased mtDNA gene expression and copy number in PD61 ${ }^{\text {NDI1 }}$ compared to PD61. This suggests that cells responded to the increases in OXPHOS activity by increasing mitochondrial biogenesis, which would lead to increased mtDNA gene expression and copy number. We did observe that PGC-1 $1 \alpha$ expression was also increased in PD61 ${ }^{\mathrm{NDI1}}$, indicating that the biogenes is pathway was in fact up regulated in response to the expression of Ndi1. The mechanism behind the coordination of increased respiratory function with an increase in mitochondrial biogenesis signaling is unclear. One possible explanation is that there was retrograde signaling of mitochondria back to the nucleus in order to increase mitochondrial biogenesis (Picard et al., 2013). Several mitochondrial-re lated pathways, such as free radicals and AMP/ATP ratios, regulate PGC-1 $\alpha$ expression (Wenz, 2011). Interestingly, sirtuins have emerged as an important class of proteins involved in the coordination of metabolism and aging (Guarente, 2007). Previous studies of PD cybrid cells reported decreased levels of PGC-1 $\alpha$ and SIRT1 (silent mating type information regulation 2 homolog) compared to control cybrid cell lines (Esteves et al., 2010). SIRT1 is a NAD-dependent deacetylase sirtuin that activates PGC-1 $\alpha$ in response to higher cytosolic NAD+/NADH ratios. Changes in the mitochondrial NAD+/NADH ratios as a result of increased NADH oxidation by Ndi1 could explain the increases in PGC-1 $\alpha$ signaling. However, a previous study in Drosophila found that expression of Ndi1 did not increase the activity of the SIRT1 
homolog, Sir2 (Sanz et al., 2010). Further studies using this model will be targeted at uncovering the mechanism behind increased PGC-1 $\alpha$ signaling in Ndil expression cells. Increasing mitochondrial biogenesis has been studied as a possible therapeutic target for PD, especially in light of the findings that PD patients had reduced PGC-1 $\alpha$ regulated gene expression (Zheng et al., 2010). As mentioned in Chapter 3, therapies targeted at increasing PGG- $1 \alpha$ have shown some promise in patient cells and models of mitochondrial disease (Bastin et al., 2008; Srivastava et al., 2009; Wenz et al., 2008). Although we observed significant changes in mtDNA gene expression, mtDNA gene copy number, and mitochondrial biogenesis gene expression, we saw a less consistent effect on the assembly of the ETC complexes in PD61 ${ }^{\mathrm{NDI1}}$. In PD61 ${ }^{\mathrm{NDI} 1}$, only CIII and CIV showed increased assembly compared to PD61. The lack of increased CI assembly was not surprising. PD61 ${ }^{\mathrm{NDI1}}$ produces high levels of the Ndi1 prote in functionally bypassing CI; therefore, additional assembly of this complex is unnecessary. Also Ndil expression only bypassed CI and did not repair the inherent dysfunction. Furthermore, any functional deficits, potentially arising from PD patient mtDNA mutations or deletions, in other ETC complexes would not be resolved by Ndil expression. It is reasonable for CIII and CIV to increase since these complexes are downstream of the increased electron transport generated by Ndil. Although we observed increases in mtDNA-encoded CI and CIV subunits, this did not necessarily translate into increased assembly of ETC comple xes. A recent study found that ETC subunits that are not incorporated into assembled complexes may be quickly de graded (Zurita Rendon and Shoubridge, 2012). If new complexes are not be ing assembled, then increases in subunit gene expression may not have an effect on the expression of assembled ETC complexes. 
This could explain why an increase in mtDNA gene expression was observed without a cons istent effect on ETC complex assembly. Future studies will be necessary to investigate if there were other functional consequences of Ndil expression, such as changes in CIV activity, heteroplasmy, or mtDNA de letions, as these are all relevant to PD.

The expression of Ndil in the SY5Y cells also resulted in some functional changes and increased biogenesis signaling. Unlike PD61, SY5Y cells are not functionally compromised. Therefore, we were surprised that there was a functional effect from Ndi1 on OXPHOS. SY5Y cells are derived from a tumor cell line, suggesting that they may rely more on glycolys is and lactic acid fermentation rather than glycolysis coupled with OXPHOS (Warburg, 1956). Studies in undifferentiated SY 5Y cells show that they rely more heavily on glycolys is than differentiated SY5Y cells, suggesting that the respiratory phenotype of SY5Y changes as cells become post-mitotic (Schneider et al. , 2011). Interestingly, a recent study found that in the presence of low glucose or galactose, SY 5Y cells had increased OCR rates, likely due to a shift from reliance on glycolys is, to using more OXPHOS for ATP production (Swerdlow et al., 2013). The changes we observed in SY $5 Y^{\mathrm{NDI} 1}$ respiratory function may indicate that the presence of Ndil led to a shift towards more use of OXPHOS. This shift could also lead to the increases we observed in ETC complex assembly, as the need for these proteins was increased. Importantly, a shift in respiration can also explain the increase in coupling efficiency in SY5Y $\mathrm{Y}^{\mathrm{NDI} 1}$ compared to SY5Y cells, as more oxygen consumption is coupled to ATP production. This is an interesting phenomenon that warrants further 
study, in particular on the effect of Ndi1 expression on mitochondrial respiration in other tumor cell lines.

As mitochondrial dysfunction is not the only pathology observed in PD cells, we also determined to effect of Ndi1 expression on levels of aggregated proteins and CLB formation. There was a decrease in cellular aggregated protein levels in PD61 ${ }^{\mathrm{NDI1}}$ but no change in CLB expression. This could be due to one of two processes: (1) expressing Ndi1 is not sufficient to prevent CLB formation or (2) expression Ndil is not sufficient to induce clearance of CLB. Cells divide and presumably the CLB can go into either daughter cell (Rujano et al., 2006), therefore the length of time since the CLB formation occurred is difficult to determine without long-term microscopy. However, inclusion of Ndil prote in into CLB indicates that at least a proportion CLB were formed following Ndil expression. This finding indicates that improving mitochondrial function alone was not sufficient to prevent the addition of dysfunctional mitochondria and proteins to the CLB. The Ndil found in the CLB colocalized with porin, suggesting that Ndil is not aggregating in the cytoplasm and then being trafficked to a CLB. It is more likely that Ndil is being incorporated into dysfunctional mitochondria that are en route to the CLB. A loss of mitochondrial membrane potential often precedes mitochondrial degradation via mitophagy (Twig et al., 2008b). As Ndil does not pump protons, and cannot contribute to the mitochondrial membrane potential, it is possible that Ndil is being incorporated into mitochondria that have reduced membrane potential. If this were the case, it would explain why Ndil is ending up in CLB. The contribution of dysfunctional mitochondria and mitophagy to CLB formation in PD will be instigated further in the next chapter. 
Electrons can leak off of CI before passage to coenzyme Q (Barja and Herrero, 1998). As mentioned in the introduction, published evidence has shown that oxidative damage to aSYN that could lead to misfolding and aggregation (Shaikh and Nicholson, 2008). Previous studies have shown that oxidative stress is reduced after Ndil expression (Park et al., 2007; Seo et al., 2006), although those studies were performed in cells that only had a CI dysfunction, unlike the PD cybrids where there is more widespread mitochondrial ETC dysfunction (Cronin-Furman et al., 2013; Keeney et al., 2009). It is possible that in our model, we may have reduced production of free radicals from CI, but there may have been an increase in free radical production from other ETC sites, such as CIII. This could increase due to the change in volume of electrons passed along the ETC. If we did not reduce cellular free radical production, then this could be the reason CLB expression did not change. The total cellular aggregated protein content, as measured by Congo red was significantly reduced, suggesting that there may have been some improve ments in the activity of proteolytic pathways, such as autophagy, driven by the increases in ATP production. It is possible that since the number of small Congo red positive cytoplasmic aggregates was decreased, we may have increased the sequestration and transfer of these aggregates to CLB, via autophagy. This study does show that improving mitochondrial respiratory function with Ndi1 was not sufficient to prevent CLB formation. In light of our results, it is like ly that an additional intervention may be necessary to improve degradation of aggregated proteins and prevent CLB formation.

One drawback to these findings is that we only transfected two lines in this study. We selected a PD cybrid cell line with prototypical mitochondrial dysfunction, abnormal mitochondrial morphology, and aSYN aggregation. The origins of the mitochondrial 
dysfunction in the cybrid cell lines are from mtDNA of a PD patient. Therefore, this was a preliminary study to test if Ndil was effective at improving mitochondrial respiratory function and reducing protein aggregation. In light of these findings, future studies will be designed include the other PD cybrid cell lines, specifically the cell lines with a range of CI function in order to expand these results. We expect there may be some heterogeneity in the effect of Ndil between the individual PD cybrid lines, due to the differences in mitochondrial function and mtDNA among these cell lines (Cronin-Furman et al., 2013; Keeney et al., 2009).

In summary, expression of Ndil in a cell model leads to improved mitochondrial function and morphology. Mitochondrial gene expression, copy number, and biogenes is were also all increased in our PD cybrid cell line after expression of Ndi1. Although we saw these mitochondrial improvements and reduced cellular aggregated prote in content, Ndil expression did not prevent the formation of CLB in the PD cybrid cell line. This finding suggests that supplementing CI with Ndi1 is sufficient to increase mitochondrial function but additional interventions may be necessary to prevent accumulation of aggregated proteins in CLB. These findings were consistent with other reports that showed Ndi1 was capable of improving mitochondrial function in other models of CImediated disease (Barber-Singh et al., 2010; Barber-Singh et al., 2009; Marella et al., 2008; Mare lla et al., 2010; Seo et al., 1998). Importantly, this study also found that Ndi1 expression lead to increase mitochondrial bioge nesis signaling, suggesting that Ndi1 warrants further research as a potential therapy for PD and other mitochondrial diseases. 


\section{Autophagy is essential for the maintenance of mitochondrial function and contributes to CLB formation in PD cybrid cell lines.}

\section{Introduction}

The presence of LB in the SNpc is used as a neuropathological hallmark for the diagnosis of PD. The mechanism behind the formation of LB is still unclear. Autophagy has been implicated as a candidate pathway due to several findings. Evidence of autophagic degeneration has been observed in nigra of PD patients (Anglade et al., 1997). Several different proteins related to autophagy have also been observed in LB (AlvarezErviti et al., 2010; Chu et al., 2009; Wakabayashi et al., 2007; Zatloukal et al., 2002). As mentioned in the Introduction, it has also been shown that autophagy plays a role in the degradation of aSYN, both the monomeric and the aggregated forms (Huang et al., 2012; Lan et al., 2012; Lee et al., 2013; Winslow et al., 2010). Autophagy is also essential for the removal of dysfunctional mitochondria, via mitophagy (Lemasters, 2005). Therefore, it is important to understand what roles autophagy plays in the mitochondrial dysfunction, accumulation of aggregated proteins and CLB formation characteristic of PD cybrid cell lines.

Autophagy can be induced by a variety of stimuli, including amino acid starvation, glucose deprivation, and oxidative stress (Bhaskar and Hay, 2007). Cellular prote ins and organelles may also need to be degraded due to dysfunction. Autophagy is initiated by the inhibition of the prote in kinase mTOR (Noda and Ohsumi, 1998). During the process of autophagy, cargo is isolated from the cytoplasm into autophagosomes, which then fuse with lysosomes to receive the lysosomal hydrolytic enzymes (reviewed in Yang and Klionsky, 2009). The result of this fusion is an autolysosome. The lysosomal enzymes degrade the contents of the autolysosomes. Autophagy is critical for 
both the maintenance of normal cell function and responding to stressors (Yang and Klionsky, 2009). Mitophagy is a specialized form of autophagy that is especially critical for cell function because it facilitates the turnover of dysfunctional mitochondria (Lemasters, 2005). Reduced mitophagy can lead to increases in oxidative stress and decreases in energy production (Lemasters, 2005). The contribution of autophagy and mitophagy to cellular homeostas is in the context of PD is an important area of study in light of the mitochondrial dysfunction and accumulation of aggregated proteins into LB that is observed in PD patients.

Prior to the discovery of CLB in PD cybrids, there was no live cell model of LB formation that did not require overexpression of nuclear genes or inhibition of protein degradation (Junn et al., 2002). Most of what is known about LB formation has been obtained from immunocytochemical and EM identification of the contents of LB, which include proteins related to UPS and autophagy, and the potential pathways involved in aSYN degradation (Alvarez-Erviti et al., 2010; Shults, 2006; Zatloukal et al., 2002). Several models have attempted to generate LB in vitro by inducing aSYN aggregation (Tanik et al., 2013; Watanabe et al., 2012). The model described by Tanik et al. was created by introducing pre-formed aSYN fibrils into ne uronal and non-neuronal cells that overexpressed aSYN (Tanik et al., 2013). The pre-formed fibrils "seed" the formation of larger aggre gates. This model is unique because it does recapitulate several of the characteristics of LB found in PD brain that other models do not, such as staining for proteasomal and autophagy related components in the LB-like inclusions (Tanik et al., 2013). However, these aggregates still required the introduction of aSYN fibrils into aSYN overexpressing cells to seed the formation of the LB-like inclusions. Although this 
model creates a similar structure to LB, it does not provide insight into the normal cellular mechanis m behind LB formation in PD brain. As discussed earlier, the CLB formed in the PD cybrid cells have the same composition as LB of PD brain, do not require aSYN overexpression, and provide us with the unique ability to study LB in livecells (Trimmer et al., 2004). Using this model, we hope to expand our understanding about the contribution of autophagy to LB formation.

In addition to LB formation, mitochondrial dysfunction is also a pathogenic feature of PD with links to autophagy, in part because mitochondrial are recycled by mitophagy (Lemasters, 2005). Our results in Chapter 4 suggested that improving mitochondrial function was not sufficient to reduce CLB formation. Since mitophagy is critical for the maintenance of normal mitochondrial function (Twig et al., 2008b), it is important to clarify the contribution of autophagy to the formation of protein aggregates and CLB in PD cybrids.

Several studies have provided insights into the links between autophagy, mitochondrial dysfunction, and the formation of inclusions similar to LB (Chu et al., 2007; Watanabe et al., 2012; Xiong et al., 2011; Xiong et al., 2013). Protein degradation by autophagy is an ATP dependent process; therefore, a decline mitochondrial function due to aging or disease can reduce proteolytic efficiency (Martinez-V icente et al., 2005; Plomp et al., 1989; Plomp et al., 1987). Also, studies of PINK or parkin mutation show that the disruption of mitophagy clearly reduces mitochondrial function (Poole et al., 2008). Studies have shown that pharmacological inhibition of autophagy leads to an accumulation in fragmented mitochondria (Terman et al., 2003). One explanation for these findings is that disruption of mitophagy results in the retention of dysfunctional 
mitochondria in the cytoplasm. Furthermore, induction of autophagy can protect cells from rotenone toxicity, presumably by clearing dysfunctional mitochondria (Xiong et al., 2011). This suggests an essential interaction between autophagy and maintenance of mitochondrial function via the clearance of dysfunctional mitochondria.

Autophagic function has not previously been studied in PD cybrids. We expect mitophagy will play a critical role in the maintenance of mitochondrial function, as described above. If the observed mitochondrial dysfunction in the PD cybrids is exacerbated by reduced autophagic efficiency, then we would expect that inhibition of autophagy will further compromise mitochondrial function. Expression of Ndi1 improved mitochondrial function and reduced aggregated protein content, potentially by increasing autophagy; however, we did not improve overall cell function enough to prevent CLB formation. If our studies show that autophagy plays a role in both the maintenance of mitochondrial function and CLB formation, then increasing autophagic effic iency could resolve several different aspects of PD pathology and be potential therapeutic approach for treating PD. A critical first step is to determine if autophagy is involved in CLB formation and if autophagy plays a role in mitochondrial quality control in PD cybrid cells.

For this study, we inhibited the turnover of autophagosomes using bafilomycin A1 (BAF), which inhibits the lysosomal vacuolar proton pump, prevents lysosome fusion with autophagosomes, and results in the accumulation of undigested mitochondria and cellular proteins (Sarkar et al., 2009). After BAF treatment, we measured mitochondrial respiration in both PD and control cybrids. We assessed numbers and sizes of CLB after BAF and investigated if autophagy related proteins were found in CLB. We also used 
live cell imaging to determine if autophagosomes were trafficked to CLB in the presence or absence of BAF. We observed that treatment with BAF reduced mitochondrial function. We also found that autophagy proteins and autophagosomes were found in CLB, even in the presence of BAF, suggesting that autophagosome trafficking to CLB is independent of lysosomal fusion. These studies clearly show that autophagy plays a critical role in maintenance of mitochondrial respiratory function and CLB formation in PD cybrids.

\section{Methods}

\section{Immunocytochemistry, Congo red staining, and Cellular respiration}

Methods performed as described in General Methods (Chapter 2).

\section{BAF treatment}

Cultures of PD and control cybrid cell lines (see General Methods) were treated with BAF (Sigma) for 24 hours at $25 \mathrm{nM}$ to induce an accumulation of autophagosomes. For electron microscopy, cells were grown in T75 flasks with normal GM with $25 \mathrm{nM}$ BAF for 24 hours before fixation in $2 \%$ paraformaldehyde and $2.5 \%$ glutaraldehyde, as described in the General Methods section. For all confocal microscopy experiments, cells were plated in $35 \mathrm{~mm}$ dishes for 48-72 hours before 24-hour treatment with BAF or vehic le. After 24 hours, PD and control cybrid cells were either transferred to clear GM or fixed in $4 \%$ paraformaldehyde. Immunocytochemistry and Congo red staining was done as previously described in the General Methods. For cellular respiration experiments, cells were plated in 24 well plates from Seahorse Biosciences for 2 hours before addition of BAF or vehicle. The timing of these experiments was set up in such a 
way that respiration measurements would fall at 24 hours post-BAF treatment. All respiration experiments were run in the presence of $25 \mathrm{nM} \mathrm{BAF}$.

\section{LC3-GFP expression}

For live cell expression of LC3-GFP, cells were plated in 35mm dishes and allowed to settle for approximately 6 hours. LC3-GFP or LC3(G120A)-GFP viral vectors (Life Technologies) was added, with a multiplicity of infection (MOI) of 25, and cells were left in media containing the vector overnight. After 18 hours, the media was changed to normal GM and cells were grown for an additional 48 hours before BAF treatment for 24 hours. Cells were switched to clear GM at 24 hours and imaged in the presence of BAF.

\section{Mitochondrial morphology}

Cells were stained using an antibody for ATP synthase (CV- $\alpha)$ according to immunocytochemistry protoc ol described in the General Methods. Ten images of each cell line and treatment condition were captured. Cell number was counted as well as the number of cells that contained fragmented mitochondria for each image, with at least 150 cells per cell line and treatment counted. Percent change was calculated by dividing the total number of cells with fragmented mitochondrial by the cell count total for each cell line.

\section{Results}

Treatment with BAF results in accumulation of autophagosomes 
The cytoplas mic distribution of autophagosomes in vehicle and BAF-treated cybrid lines was measured using electron microscopy, immunocytochemistry, and live 


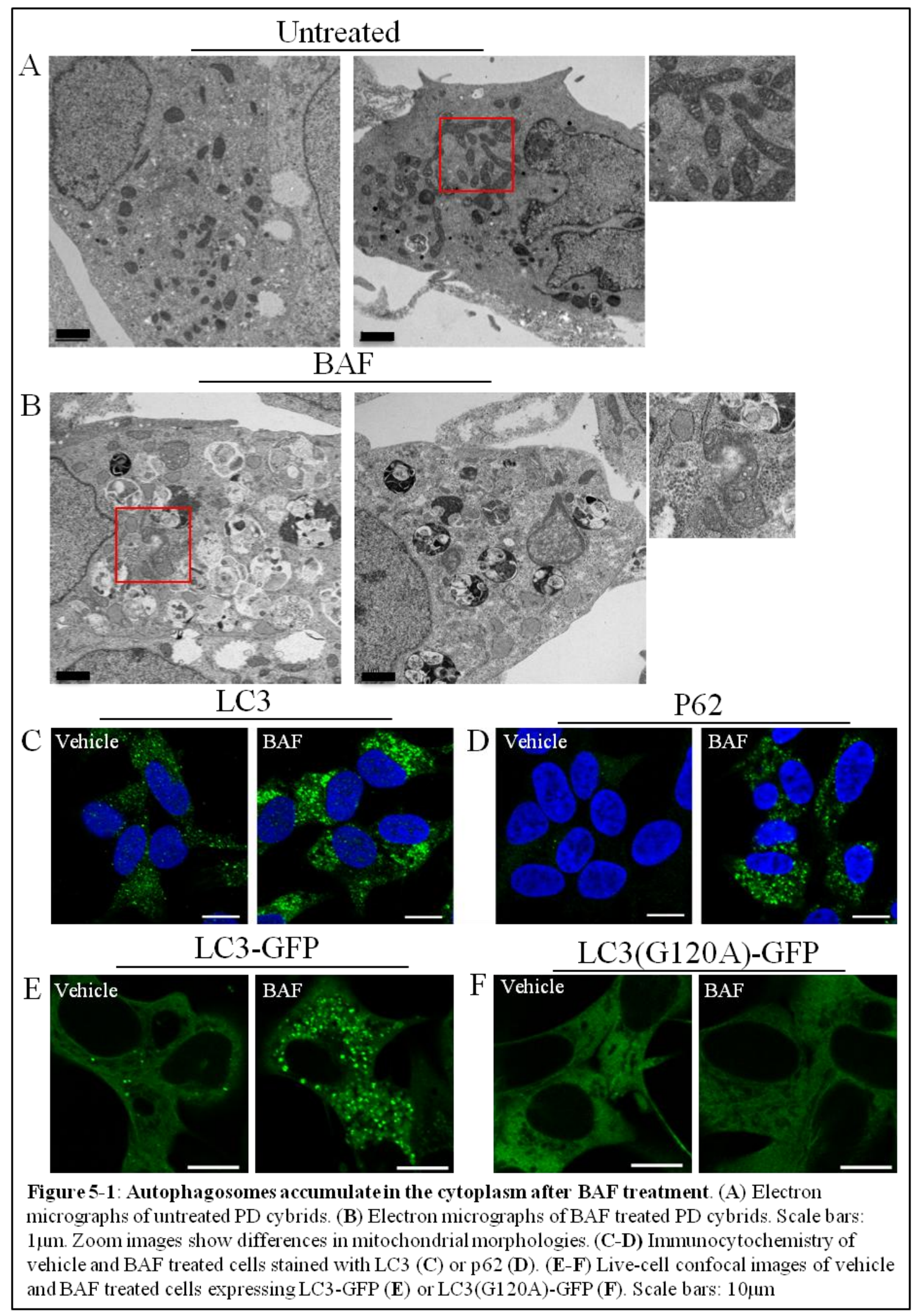


confocal microscopy. At the EM level, autophagosomes were identified as membranebound vesic les with a double (or multiple) limiting membranes containing cytoplasm and organe lles (Eskelinen, 2008). In all three PD cybrid cell lines imaged (PD61, PD63, PD67), there was a dramatic increase in autophagosomes after treatment with BAF compared with the vehic le treated cells (Figure 5-1A, B), similar to what has been observed in neurons (Boland et al., 2008). Figures 5-1C and 5-1D show the cytoplasmic punctate staining pattern of two markers of autophagy, LC 3 and p62, which accumulated after BAF treatment in all cell lines imaged. Hydrolytic lysosomal enzymes normally degrade LC3 and p62 after autophagosomes and lysosomes fuse (Kabeya et al., 2000; Pankiv et al., 2007). In the absence of this fusion, LC3 and p62 will remain in the cytoplasm, associated with autophagosomes.

Live confocal microscopy was also used to visualize cytoplasmic autophagosome distribution in BAF and vehicle treated cell lines. We used a viral vector (BacMam 2.0, see Methods) to express a LC3-GFP construct in two of our PD cybrid cell lines, PD61 and PD63. These lines were selected for transfection because both PD61 and PD63 have well characterized mitochondrial dysfunction (Cronin-Furman et al., 2013; Keeney et al., 2009). After transfection with the LC3-GFP construct, the cytoplasmic distribution of LC3-GFP was consistent with the immunocytoche mistical staining pattern of LC3 across both cell lines (Figure 5-1C, 5-1E). In vehicle-treated cell lines, there was very light punctate LC3 staining (Figure 5-1E). However, BAF-treated PD cybrid cells, there a substantial increase in the amount cytoplasmic LC3-GFP staining (Figure 5-1E).

In order to confirm that this is functionally relevant autophagos ome-bound LC3, we also expressed a mutant form of LC3, LC3(G120A)-GFP, in the two PD cybrid cell 
lines. This mutation results in a prote in that is not lipidated and, therefore, not recruited to autophagosome membranes (Kabeya et al., 2000). The cytoplasmic distribution of LC3(G120A)-GFP construct was diffuse, without any punctate staining, making it notably different from the wild type LC3-GFP in both the vehicle and BAF treated cells (Figure 5-1F). Taken together, these results using different imaging methods all consistently showed that $25 \mathrm{nM} \mathrm{BAF}$ for 24 hours is sufficient to induce the accumulation of autophagosomes.

Inhibition of autophagy decreases mitochondrial respiration and leads to fragmented mitochondrial morphology

To determine if inhibiting autophagy affects mitochondrial function, cellular respiration (OCR) was measured using the Seahorse XF analyzer. Treatment with BAF resulted in significant and consistent decreases in OCR across 5 PD cybrid cell lines and 4 control cybrid cell lines (Figure 5-2A, B). Basal, ATP-linked, maximum capacity, and complex I-linked OCR was decreased in all of the BAF-treated PD and control cybrids (Figure 5-2C, D). Interestingly, we did not see any consistent increase basal ECAR rates in either the PD or control cybrid cell lines, suggesting the cells have not shifted towards higher levels of glycolysis to compensate for the decline in OCR after $24 \mathrm{hr}$ BAF treatment. This could be due to the time frame; treating cells chronically with BAF may be necessary for cells to shift towards more dependence on glycolysis.

The dramatic decline in OCR after BAF in the control cybrids was surprising. Previous studies have shown that the PD cybrids used in this study all have varying levels of mitochondrial dysfunction (Cronin-Furman et al., 2013; Keeney et al., 2009); therefore, we expected that inhibiting turnover of dysfunctional mitochondrial by treating 
with BAF would further reduce mitochondrial respiratory rates in the PD cybrid cells.

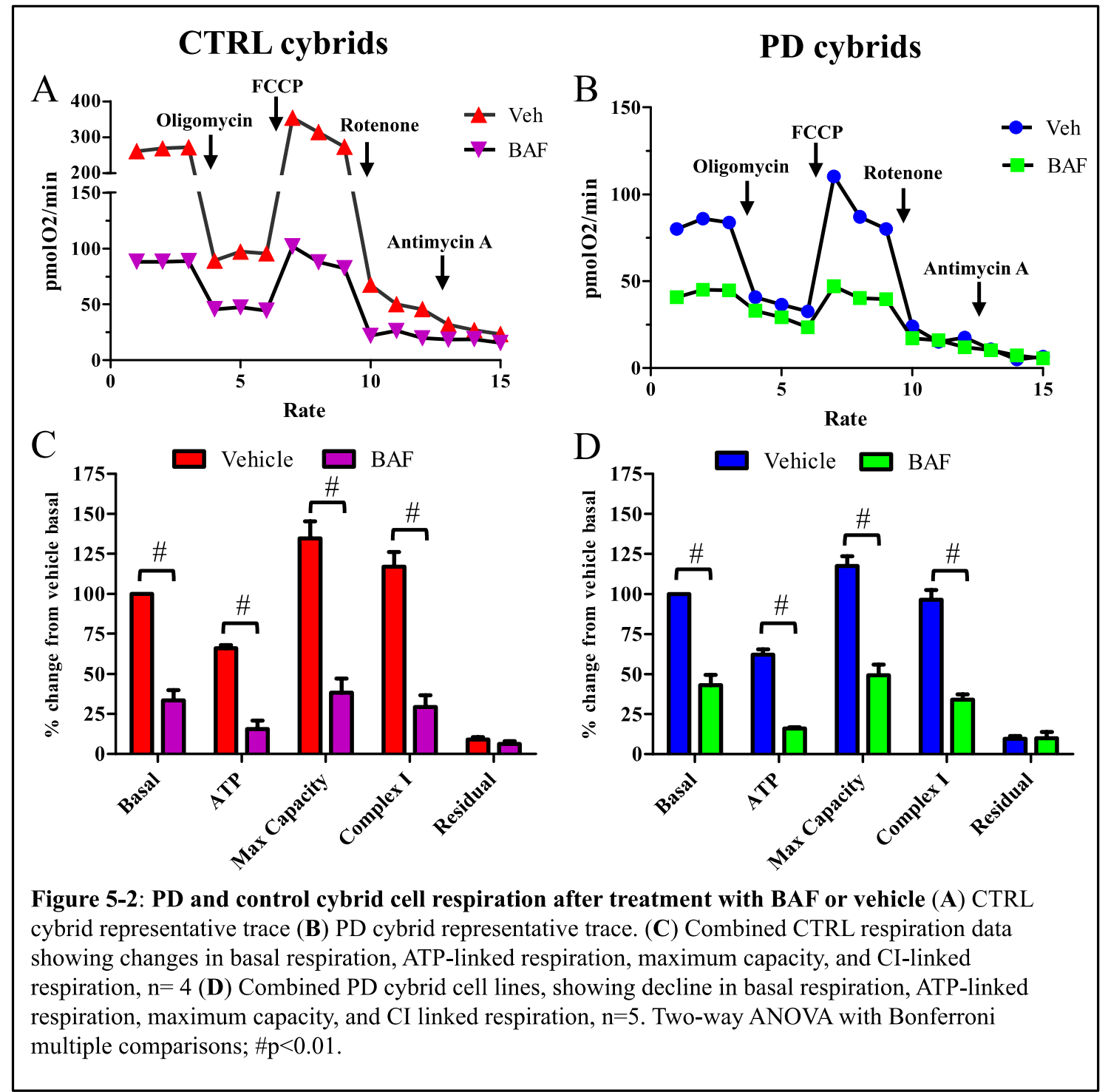

However, our results us ing BAF showed that the control cybrid cell lines also require autophagic function to maintain normal mitochondrial respiration.

Mitochondria exist in cycles of fission and fusion. Mitochondria that have undergone fission, often due to a loss of normal mitochondrial membrane potential, can then be degraded via mitophagy (Twig et al., 2008b). Inhibition of autophagosome turnover with BAF could result in reduced clearance of dysfunctional fragmented mitochondria that would normally be degraded via mitophagy. To determine if this 
occurred, mitochondrial morphology was characterized in 2 PD cybrid cell lines (PD61 and PD65) and a control cybrid cell line (CTRL68). These two PD lines were selected because they represent the range of mitochondrial function observed in the PD cybrid cells. There was a 14-16\% increase in BAF-treated cybrid cells containing fragmented mitochondria in the BAF treated cells compared to vehic le (Figure 5-3A, B). This percent change was consistent across both PD cybrid cell lines and the control cybrid cell line.

An increase in fragmented mitochondria after autophagy inhibition has been previously reported (Terman et al., 2003). This suggests that mitochondria that have undergone fission. This increase in fragmented mitochondria correlates with the decline in OCR in both the PD and control cybrid cells. The fragmented mitochondria may be less effic ient at respiration due to a loss of mitochondrial membrane potential. However, it is important to note that we observed a $50 \%$ or more reduction in basal respiration in both the PD and the control cybrid cell lines (Figure 5-2C, D), suggesting that the 14$16 \%$ increase in fragmented mitochondria cannot explain all of the mitochondrial dysfunction. This deterioration of mitochondrial morphology and loss respiratory function in both PD and control cybrids after BAF supports our idea that autophagy plays a key role mitochondrial quality control.

\section{Inhibition of autophagy incre ases aggregate d protein content}

In light of the increase in cytoplasmic autophagosomes after BAF treatment, we visua lized cellular aggregated prote in content using Congo red in 5 PD cybrids and 4 control cybrids. Inhibition of autophagy with BAF resulted in a significant and consistent increase in the small prote in aggregates in both PD and control cybrids (Figure 5-4A-C). 
This increase in

Congo red

positive small

aggregates could

contribute to CLB

size and

expression;

however, there

was no difference

in size or number

of CLB between

the BAF-treated

and vehicle-

treated PD

cybrids. One

explanation is that

this may be due to

the short time

frame (24 hours)

of this set of

experiments.

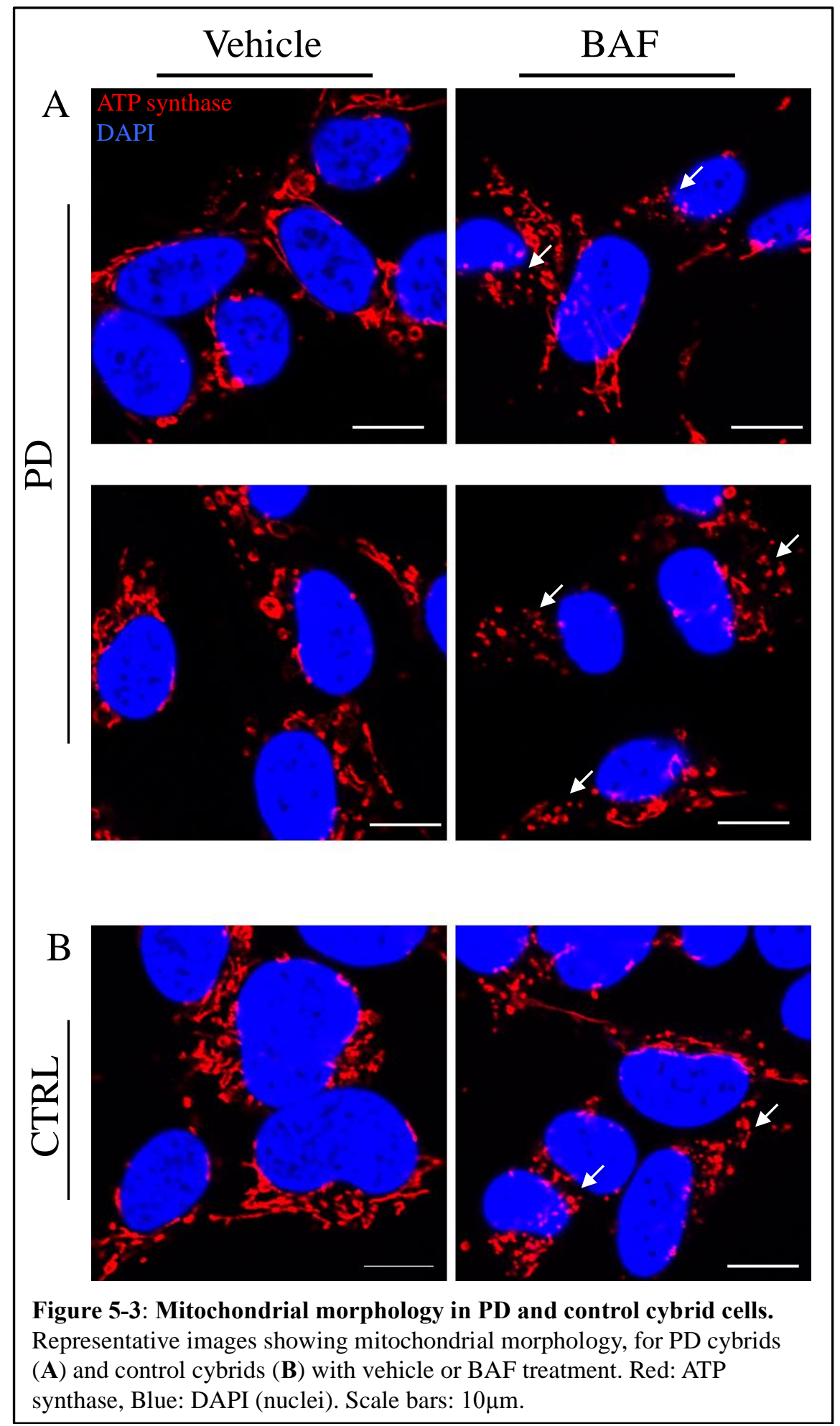

Future studies will be necessary to determine if chronic autophagic inhibition with BAF will lead to increase CLB size or, potentially, an increase in CLB frequency. 


\section{Autophagy components are found in CLB}

Previous studies have identified several autophagy related proteins in LB, such as LC3 and LAMP2A in post mortem PD patient brain (Alvarez-Erviti et al., 2010; Chu et al., 2009). Both of these proteins are involved in various steps of the autophagic process. LC3 is bound to autophagosomes and present during autophagos ome initiation, formation, and engulfment of cargo prior to lysosomal fusion. LAMP2A is found in the lysosomes and autolysos omes. Therefore, we visualized LC3 and LAMP2A staining in CLB from PD cybrids. Using immunocytochemistry, LC3 and LAMP2A staining was observed in cytoplasm and CLB from PD cybrids (Figure 5-5A, B). These images confirm that LC3 is incorporated into the CLB, but they do not illustrate if the LC3 is in autophagos omes, autolysosomes, or both.

The PD cybrid cell model has provided us with a unique opportunity to study CLB dynamics in live cells. After staining with LC3-GFP and Congo red, we imaged LC3 and CLB in real-time. In the vehicle treated cells, there was heterogeneity in the incorporation LC3-GFP into the CLB. 51\% of CLB contained LC3-GFP (Figure 5-5C); however, in 49\% of CLB there was no detectable LC3-GFP (Figure 5-5D). The LC3GFP in CLB colocalized with Congo red staining (Figure 5-5C). This could indicate that autophagos ome-bound LC3 is trafficked to and incorporated into the CLB, as part of CLB formation. Another possibility is that LC3-GFP is being overexpressed and aggregating independently of a functional role, as has been described in other studies (Kuma et al., 2007). Overexpressed exogenous proteins can be trafficked to aggresomes, so it is important to determine if the LC3-GFP trafficking to CLB was functional or not. 
Therefore, we also investigated if the mutant form of LC3 was incorporated into CLB.

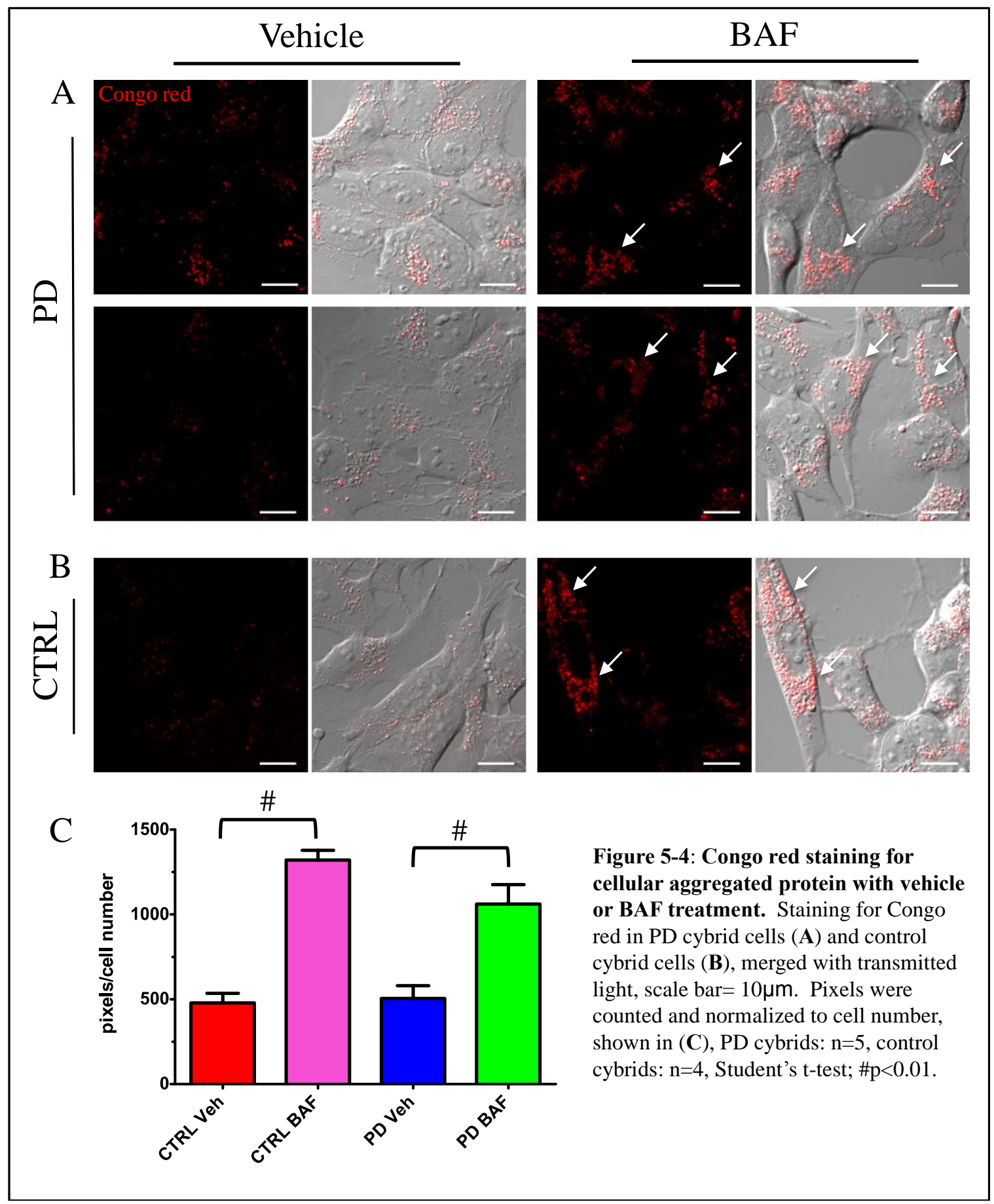

There was a small proportion (less than $20 \%$ ) of CLB that did contain some

LC3(G120A)-GFP. The inclusion of the mutant protein into a CLB is consistent with a previous study that showed this construct can associate with protein aggre gates created 
by inhibiting autophagy and the UPS (Wang et al., 2013). However, there was a higher

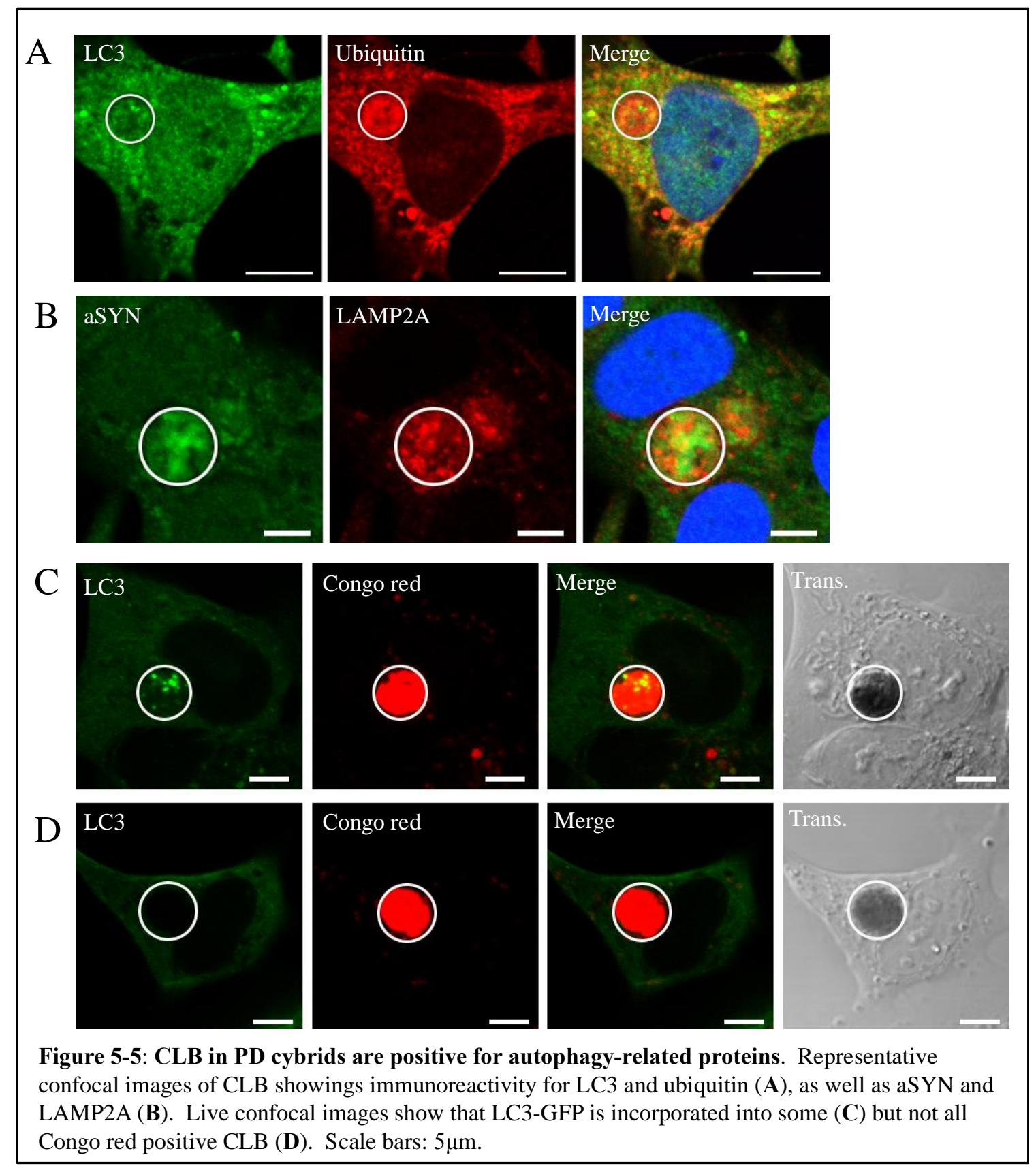

frequency of CLB with wild-type LC3-GFP (51\%) than the mutated form (less than

$20 \%$ ), indicating that the presence of LC3-GFP in CLB is related to autophagic function.

This suggests that LC3 is trafficked to CLB in in the PD cybrids as part of a functional role for autophagy in CLB formation or growth. 


\section{Autophagosomes ass ociate with CLB independent of lys osomal fusion}

The presence of both lysosomal and autophagosomal prote ins in LB and CLB suggests that autophagosomes, lysosomes, and potentially even autolys osomes are found in LB and CLB. It is currently unknown if autophagosome and lysosomal fusion is required for addition of autophagosome contents into CLB. If autophagosome and lysosomal fusion is required for addition of components to CLB, then BAF treatment should prevent the incorporation of autophagosomes into CLB. In order to test this, the autophagos ome distribution in and around CLB of BAF treated PD cybrids was examined us ing EM and live confocal imaging.

EM of PD cybrids showed that there was a difference in ultrastructure of CLB after BAF treatment (Figure 5-6A, B). The CLB found in untreated PD cybrids contain a highly e lectron dense core with surrounded by an area that is less electron dense. This outer area, which has uneven edges with variations in electron density, may also contain visible autophagos ome me mbranes (Figure 5-6A). The CLB in BAF treated cells also had a dense core (Figure 5-6B). However, there were also visible double-membrane enclosed compartments resembling autophagosomes that appeared to be integrating into the CLB (Figure 5-6B, arrowheads). The contents of these compartments were markedly less electron dense than the rest of the CLB and the edges of CLB in untreated cells. The low density of these compartments makes it likely that they are early autophagosomes. Late autophagos omes are thought to become more electron dense as components begin to degrade over time or are broken down by lys osomal enzymes (Eskelinen, 2008). This 
suggests that newly formed autophagosomes can be incorporated into the CLB in the presence of BAF before fusion with lysosomes.
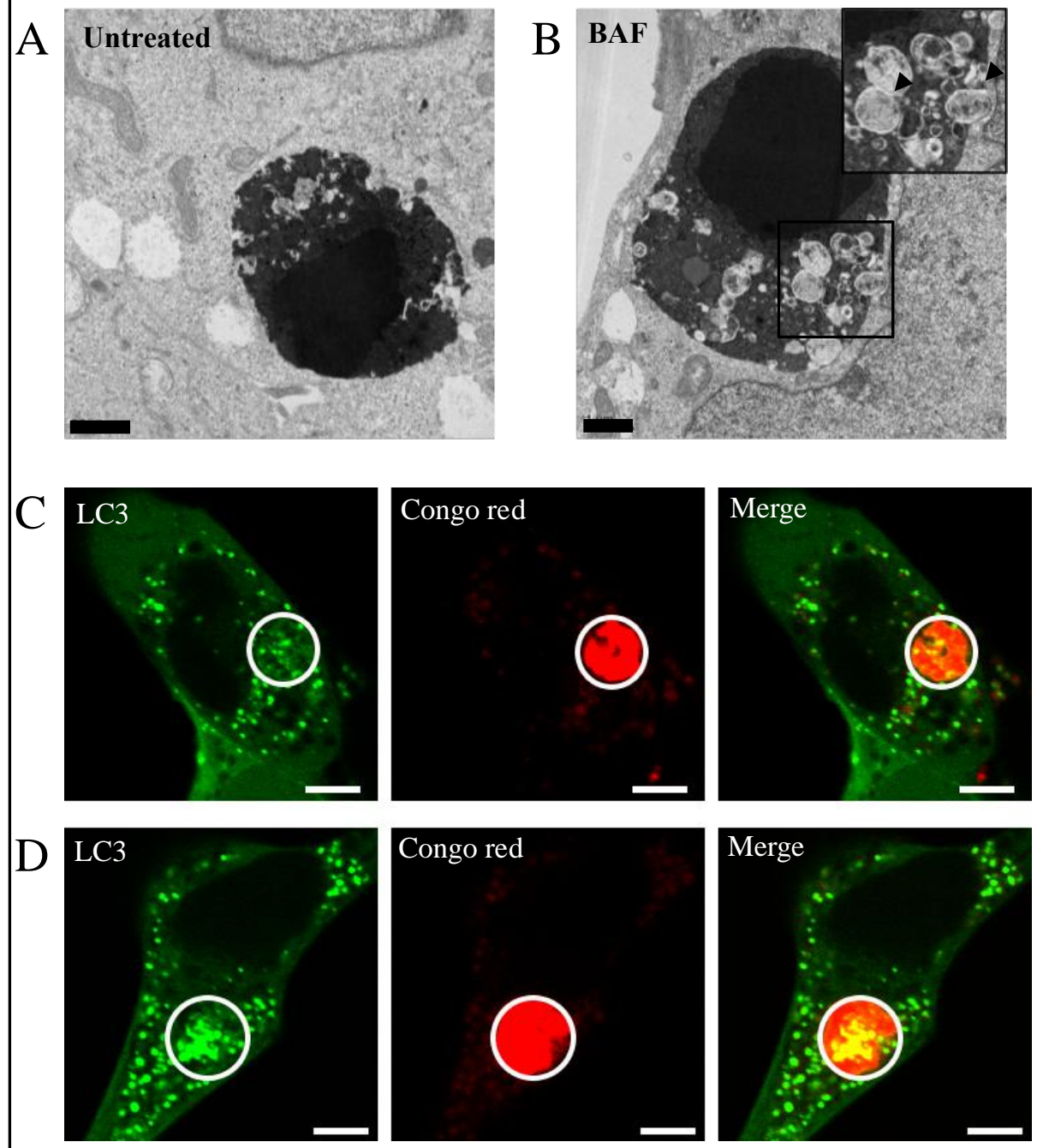

Figure 5-6: Autophagosomes are present in CLB of cybrid cells after BAF treatment. (A, B) Representative electron micrographs of CLB with or without BAF treatment. The CLB from the BAF treated cells had more early autophagosomes than the untreated cells (see high mag inset). Scale bar: $1 \mu \mathrm{m} .(\mathbf{C}$, D) Representative confocal images of LC3-GFP incorporation in the Congo redpositive CLB after BAF treatment. Scale bar: $5 \mu \mathrm{m}$.

To further characterize the distribution of autophagosomes to CLB, we imaged the CLB found in BAF treated cells expressing LC3-GFP (Figure 5-5C, D). Similar to the vehicle-treated cells described above, there was heterogeneity in the inclusion of 
LC3-GFP into CLB in BAF treated cells. To compare the inclusion of LC3-GFP into CLB in the vehicle versus BAF treated cells, we acquired images of at least 25 CLB from two different cell lines and compared the frequency of LC3-GFP labeling. Using this approach, we found that there was a higher frequency (79\%) of BAF treated cells with LC3-GFP in CLB compared to the vehicle treated cells, which only had 51\% of CLB containing LC3-GFP (Figure 5-5C, D). Furthermore, there was no difference in the amount of LC3(G120A)-GFP in CLB between vehicle and BAF treated cells. This suggests that the increase in LC3-GFP in CLB of BAF treated cells is due to autophagos ome trafficking to CLB. Similar to the electron microscopy results, this finding also indicates that autophagosomes can be integrated into the CLB and this process was increased by short-term treatment with BAF.

\section{Discussion}

This study investigated the contribution of autophagy to mitochondrial function in the PD and control cybrid cells, as well as to CLB formation in PD cybrid cells. Inhibition of autophagosome and lysosome fusion with BAF significantly reduced mitochondrial respiratory function. Treatment with BAF also led to a substantial increase in the number of cells containing fragmented mitochondria. The autophagy related prote ins LC 3 and LAMP2A were found to colocalize at CLB with ubiquitin and aSYN, suggesting an involvement for autophagy in CLB formation. Lastly, after BAF treatment, autophagosomes were observed in CLB, indicating that fusion of lysosomes and autophagosomes is not required for addition of autophagos ome contents to CLB. Mitochondria-selective autophagy, mitophagy, plays an important role in mitochondrial quality control by sequestering and degrading dysfunctional mitochondria 
(Twig et al., 2008b). For this study, we disrupted autophagic efficiency by inhibiting autophagos ome turnover. We observed an increase in cells with fragmented mitochondria and a decrease in cellular respiration (Figure 5-2 and 5-3). The abnormal mitochondrial morphology is consistent with previous reports that inhibition of autophagy by 3-MA, which prevents autophagosome formation, leads to an increase in fragmented mitochondria (Terman et al., 2003). The increase in cells with fragmented mitochondria after autophagy inhibition suggests dysfunctional mitochondria have undergone fission to order to be degraded. This could be studied further by specifically measuring if fragmented mitochondria are less functional, by looking at mitochondrial membrane potential or free radical production. Additionally, in the absence of normal autophagos ome turnover, it is possible that the cybrid cells are retaining these dysfunctional, fragmented mitochondria instead of degrading them. Chu et al. suggested that if the rates of mitophagy exceed the rate of mitochondrial biogenesis, then ATP production would decline, resulting in an energy cris is (Chu et al., 2007). In our studies, after BAF treatment, mitochondria may be sequestered into autophagosomes but are not being degraded in the absence of autophagosome and lysosome fusion. This could mean that the basic biomolecules of mitochondria are not available for reuse in the biogenes is of new mitochondria. This imbalance between mitochondrial degradation and biogenesis could then lead to the energy crisis proposed by Chu et al., if autophagic sequestration exceeds rates of biogenesis in our cybrid cell lines (Chu et al., 2007). This may explain the significant loss of respiratory function that we observed. Furthermore, this may have important re levance to PD, if dysfunctional mitochondria in PD patient neurons are not 
properly degraded and mitochondrial biogenesis cannot compensate, this could explain the loss of mitochondrial function in PD (Parker et al., 1989; Schapira et al., 1990a).

One interesting observation was that the change in number of cells containing fragmented mitochondria was consistent between three different cell lines, two PD cybrid cell lines and one control cybrid cell line. It is important to note that the cell line with the lowest basal respiration rate, PD61, did have a higher frequency of cells containing fragmented mitochondria in the vehicle treated dishes than the other cell lines; however, the change in frequency was the same after BAF treatment. We expected the cells with more mitochondrial dysfunction (i.e. PD cybrids cells) would be more dependent on mitophagy for maintenance of mitochondrial function than the control cybrid cells. Mitochondria can elongate to avoid mitophagy and maintain levels of ATP (Gomes et al., 2011). This type of adaptation may occur in cells like PD61 that have relatively poor mitochondrial respiratory function. If this has occurred, then elongated dysfunctional mitochondria could be retained in the cytoplasm under normal conditions, sparing them from mitophagy. Furthermore, these mitochondria could explain why the observed decline in respiratory function was greater than the change in the frequency of cells with fragmented mitochondria.

The significant changes in mitochondrial respiration after BAF treatment suggest that mitophagy is essential for the maintenance of mitochondrial function in the PD and control cybrid cells. It is possible that this loss of mitochondrial respiratory function is due to a direct effect of BAF on mitochondria. Previous studies have shown that BAF can inhibit respiratory function of isolated mitochondria; however, those studies used considerably higher dosages of BAF with the intent to specifically inhibit isolated 
mitochondria (Zhdanov et al., 2011). However, taken together, our results suggest that mitochondrial respiratory function is likely reduced due to the interruption in autophagic turnover and not due to an inhibitory effect of BAF on mitochondrial respiration. The loss of respiratory function also indicates that cells require normal autophagic function to mainta in normal rates of ATP production, as we expected. In this study we did not investigate if other mitochondria-related processes such as calcium buffering, mitochondrial membrane potential, or oxidative stress, were affected. In light of the changes in respiratory function and mitochondrial morphology, we would expect that long-term autophagic inhibition could have significant effects on these other mitochondrial functions. As autophagy is ATP dependent (Plomp et al., 1989; Plomp et al., 1987), improper clearance of dysfunctional mitochondria could reduce autophagic function. This vicious cycle of reduced clearance of dysf unctional mitochondria, coupled with a decline in autophagic function, could be a potential mechanism of PD pathogenesis.

In the cybrid cells it was apparent that there is an increase in autophagosomes in CLB after BAF treatment. This was not surprising given that BAF prevents autophagos ome and lysosome fusion and, as described above, leads to accumulation of autophagos omes in the cytoplasm (Sarkar et al., 2009). Prior to these studies, we did not know the mechanism of autophagosome inclusion in CLB. Although there was evidence of autophagy related proteins in LB from PD patient brain (Alvarez-Erviti et al., 2010; Wakabayashi et al., 2007; Zatloukal et al., 2002), it has never been show $\mathrm{n}$ if autophagosomes are responsible for delivery of aggregated prote ins or dysfunctional organe lles to LB. Furthermore, even though lysosomal prote ins are found in the LB, it 
was not known if autophagosomes and lys osomes fusion occurred before inclusion into a LB. It is also possible that the inclusion of autophagy-related proteins in LB is because these organelles have become dysfunctional themselves. However, we have no evidence that autophagosomes are dysfunctional in our studies.

The addition of early autophagos omes to CLB, as suggested by electron density, would indicate that autophagosomes can be incorporated into CLB without previously fusing with lys osomes. Staining for acidic compartments using lysosomotropic dyes showed that active lysosomes are present in CLB (Trimmer et al., 2004), which suggests that autophagosomes may arrive at the CLB in order to fuse with lysosomes and undergo degradation. Our results support this theory and future studies using different inhibitors of autophagy, such as lysosomal inhibitors to prevent autolysosome content de gradation, could help further elucidate how autophagosomes and lys osomes are interacting at the CLB.

LB are usually found in a perinuc lear area, and colocalize with $\gamma$-tubulin, a prote in found at the microtubule-organizing center (MTOC) (McNaught et al., 2002). Interestingly, a recent study found that lysosome subcellular location plays an important role in the autophagic response to starvation. Lysosomes are redistributed to perinuclear areas near the MTOC during starvation conditions (Korolchuk et al., 2011). The authors showed that under these conditions, lysosomal localization plays a role in coordinating mTOR activity, autophagos ome synthes is, and autolysosome turnover. This response is dependent on microtubule assembly, as treatment with microtubule destabilizers reduced mTOR activity. (Korolchuk et al., 2011). Disease conditions such as oxidative stress, reduced mitochondrial function, and increased protein aggregation could trigger this 
lysosomal redistribution and a subsequent autophagic response. If the lysosomes in PD patient cells are coordinating autophagy, then lysosomal localization to the MTOC could be an important factor in the initiation of LB formation or LB growth. This is a possible explanation of the presence of lysosomal and autophagic proteins in LB and CLB.

A recent study that showed that treatment with BAF, in cells overexpressing aSYN, formed fewer aggregates (Klucken et al., 2012), suggesting that the fusion of autophagosomes and lysosomes may participate in aSYN aggregation. This study also reported an increase in aggregate formation after rapamycin treatment, which could mean that increased autophagy can lead to more aSYN aggregation (Klucken et al., 2012). Other studies reported reduced aggregation after treatment with rapamyc in or other autophagy inducers, potentially due to the increased degradation of aggregated proteins by autophagy (Lan et al., 2012; Watanabe et al., 2012). Even though these studies used different cell culture models, aSYN overexpression techniques, and inhibitors, there are several reports in the literature support the conclusion that autophagy is relevant to aSYN degradation (Alvarez-Erviti et al., 2010; Cuervo et al., 2004; Huang et al., 2012; Lee et al., 2013; Wins low et al., 2010; Yu et al., 2009). Our studies, which do not require the exogenous introduction of aSYN to induce CLB formation, suggest that autophagosomes could be responsible for the addition of aggregated proteins and dysfunctional organelles to CLB. Our data also suggest that lysosomes may also play an essential role in the CLB expression. The differences in electron densities observed on the outer edges of CLB in vehicle versus BAF treated CLB suggest that lysosomes contribute to some partial degradation at the CLB in untreated PD cybrid cells. These studies bring up several important questions about how autophagy contributes to CLB formation, most 
importantly if autophagy is involved in the initial formation of these inclusions or just their expansion.

In conclusion, we showed that autophagy plays an integral role in maintenance of mitochondrial function and CLB formation in PD cybrid cells. This study also showed for the first time that autophagosomes are trafficked to and included in LB-like aggregates without previously fusing with lysosomes. In light of these findings, we believe that autophagy is very relevant to PD pathogenesis and is a valid therapeutic target for the treatment of PD. Improving autophagic effic iency in addition to treating mitochondrial dysfunction may be the most efficacious approach to treating a disease that features both mitochondrial dysfunction and protein aggre gation. Although the role of autophagy in LB formation in PD patients is still unclear, these studies show that there is strong evidence for the involvement of this pathway in PD. 


\section{Conclusions and Future directions}

$\mathrm{PD}$ is a motor neurodege nerative disease, affecting over 1 million patients in the United States, for which there currently is no cure. The symptoms and pathology of PD have been well described, but the exact pathogenic mechanis ms of this disease still elude us. The discovery of several genes that are mutated in the rare forms of familial PD have shed some light on several processes that are abnormal in PD, most notably mitochondrial function and protein degradation. The models created using these genes have been helpful in determining the ways that these pathways could contribute to PD pathogenes is but has not led to the development of successful therapeutics. Our lab uses an alternate approach, a cybrid cell line created from sporadic PD patients, which better reflects the heterogeneity observed between patients (Trimmer and Bennett, 2009).

Historically, mitochondrial dysfunction and LB formation have been described as two of the classical pathological hallmarks of PD, in addition to DA cell death. Frederic Lewy first described the LB in 1912. The link between mitochondrial dysfunction and PD was discovered later, when MPTP-induced parkinsonism was described (Langston et al., 1983). Our studies were designed to specifically look at the relationship between these two important pathologies. If one directly influences the other, such as mitochondrial dysfunction specifically leading to LB formation or vice versa, then modulating either mitochondrial function or prote in degradation could be the key to slowing PD pathogenesis. The presence of CLB in the cybrid model suggests that mitochondria, specifically mtDNA, contribute to protein aggregation; however, this mechanism remains unclear. Our studies described in Chapters 3 and 4 showed that although mitochondrial function affects protein aggre gation, the nature of the relationship 
between these two processes is not straightforward. Improving mitochondrial function reduced total cellular protein content, but it did not prevent CLB formation over the course of our experiments. These findings led us to look at what other pathways could be modulating both mitochondrial function and prote in aggre gation. As shown in Chapter 5, we found that the obvious "missing link" between these processes to be autophagy. A role for autophagy in PD is not a novel idea, as some of the genes mutated in familial PD are involved in protein degradation or mitophagy. The mechanism by which autophagy contributes to aggre gate formation has not been described due to a lack of models to accurately reproduce a true LB in live cells. By using the cybrid model, we were able to show for the first time that autophagosomes are incorporated into CLB, suggesting that autophagy could be a valid therapeutic target to prevent LB formation in PD patients. Is autophagy a valid therapeutic targetfor PD?

Many studies have aimed to use autophagy inducers, in particular rapamycin, a specific inhibitor of mTOR, to increase rates of autophagy in order to clear aggregated proteins from cells. The results of these studies have been mixed. In some cases, rapamycin is protective against damage from rotenone, potentially by c learing dysfunctional mitochondria more quickly and reducing oxidative stress (Xiong et al., 2011). In other cases, it has been reported that overactivation of autophagy is linked to cell death (reviewed in $\mathrm{Chu}, 2006)$. One study even found that rapamycin treatment increased aggregate formation (Klucken et al., 2012). An important drawback to using rapamycin is that it does not cross the blood brain barrier, so other small molecules that have a similar effect may be more informative (Sarkar et al., 2007). 
Increasing autophagy to improve cell function in a PD patient is an interesting concept. However, our studies suggest that timing of this therapy in disease progression may be critical for determining if this is a valid course of treatment. If we accept that PD pathogenes is involves a decrease in mitochondrial respiratory function and an increase in prote in aggregation over time, then increasing the amount of autophagy might be a dangerous thing for a cell. First of all, as described earlier, protein and organelle degradation by either the UPS or autophagy is ATP dependent. Artificially increasing autophagy w ould require additional ATP. Increasing the energy demands in cells with compromised mitochondrial respiratory function could lead to increased free radical production as dysfunctional mitochondria attempt to produce more ATP.

Instead, this type of intervention might be more efficacious in earlier stages of PD, where mitochondrial respiratory function is less compromised and the aggregated prote in load is lower. It may also be benefic ial to simultaneously improve mitochondrial function to generate more ATP. If autophagy is increased in an effort to clear the dysfunctional mitochondrial and aggre gating proteins, then this could prevent the accumulation of these proteins into LB. One concern with this type of approach is that global induction of autophagy might decrease the specificity of aut ophagy, leading to the degradation of healthy, as well as damaged or dysfunctional proteins and organelles. There is also evidence that this type of autophagy induction can overwhelm the cell, making degradation less efficient (reviewed in Cherra and Chu, 2008). Accurate titration for each patient of this type of treatment would be necessary to minimize the degradation of normal proteins and mitochondria. If optimal dosage is achieved, the result could be a 
healthier pool of mitochondria with higher production of ATP and lower production of free radicals.

One drawback is that although autophagy inducers can certainly increase autophagy, there is no comparable effect on lysosomal function. As mentioned earlier, lysosomal function declines with age and is associated with the build up of nondegradable lipofuscin (Kurz et al., 2007). Additionally, cells have only a limited ability to produce lysos omal enzymes (Brunk and Terman, 2002). Our studies suggest that lysosomes are present in the same area as autophagosomes, near the MTOC. Although autophagy may be reduced in PD patients, if lysosomal function is not also improved, then increasing autophagy may only lead to the sequestration of dysfunctional mitochondrial and proteins, without any degradation of autophagosome contents. As mentioned in Chapter 5, this could lead to an energy cris is, as mitochondria are sequestered but not degraded, and mitochondria biogenesis may not be able to regenerate the mitochondrial population (Chu et al., 2007). Our studies us ing BAF to prevent autophagos ome-lysosome fusion are an example of the possible consequences of increasing autophagy induction without improving lysosomal function. Autophagosomes were able to sequester cargo after treatment with BAF; yet, in the absence of autophagos ome turnover, mitochondrial function was significantly reduced. Can we use the PD cybrid cell model to develop autophagy-targeted therapies?

The successful development of novel and effective therapeutics also requires the use of the correct models. Our studies in Chapter 5 described the important of autophagy in the PD cybrid cells for maintenance of mitochondrial function and CLB formation. We have clearly shown that mitochondrial function and CLB formation are present in the 
PD cybrid cells, similar to what has been observed in PD patients. Additionally, our model of spontaneous CLB formation is a unique approach to studying the contribution of autophagy to aSYN aggregation, without aSYN overexpression. Therefore, we believe that the PD cybrid cell will be a valuable model for the development of autophagy-related therapies.

One important characteristic of the PD cybrid model is that the cybrid cells initiate autophagy without pharmacological induction or starvation. Many other cell models require nutrient withdrawal to induce autophagy (e.g. Sarkar et al., 2007); however, like neurons, the PD cybrid cells mainta in constitutively active autophagy. This was shown by the accumulation of autophagosomes in the cytoplasm after treatment with BAF in Chapter 5. We have also established a role for autophagy in the maintenance of mitochondrial function. This indicates that manipulation of autophagy could lead to improved mitochondrial respiratory function. As mentioned in earlier sections, reduced mitochondrial function can contribute to a decline in autophagic function (Plomp et al., 1989; Plomp et al., 1987). Our studies described in Chapter 4 showed that respiratory improve ment was not sufficient to rescue autophagy and prevent CLB formation. Future studies will attempt to improve autophagy, without directly affecting mitochondria, and then investigate if improvement of autophagy is sufficient to rescue mitochondrial respiratory dysfunction. In light of our studies, we believe that understanding the relationship between mitochondrial function and autophagic de gradation is essential for designing better therapeutic approaches.

The major drawback to the PD cybrid cells method is that these cells are mitotic; they are able to divide and, through asymmetric division, is olate dysfunctional organelles 
and aggregated prote ins into one daughter cell. However, in post-mitotic cells, such as neurons, this type of asymmetrical division is not possible. Neurons must cope with the accumulation of aggre gated proteins and dysfunctional mitochondria over time. New models will need to focus on recapitulating the heterogeneity we have achieved in the PD cybrids, while simultane ously modeling post-mitotic cells.

One of the newest approaches to modelPD is the field reverse engineered inducible pluripotent stem cells. This method involves taking blood or skin cells from a patient and reverse engineering the m into pluripotent stem cells (Takahashi et al., 2007). These cells can be differentiated in neurons, as a way to study PD and other disease in neurons derived from human patients (reviewed in Ming et al., 2011). In the future, we hope to take advantage of this technology as a means to verify our findings and aid in the development of new therapeutics for patients. In particular, if ne urons created from patient-derived cells create LB, this will be an important model for studying the contributions of autophagy to mitochondrial function and LB formation in a neuron. This model does have drawbacks; in particular there is concern that the low efficiencies of the transformation (Soldner et al., 2009) could result in unsuccessful transformation of the cells with the most compromised mitochondrial dysfunction. If this is the case, then the neurons created by reprogramming PD patient cells may not accurately represent the pathology observed in the patient. This is a quickly evolving and exciting area of research that warrants further study and will hopefully aid in the development of novel therapeutics.

In conclusion, these studies described in this dissertation using PD cybrids have advanced our understanding of PD pathogenesis. We have shown that although 
mitochondrial dysfunction and prote in aggregation are integral processes, autophagy als o plays an important role in regulating both mitochondrial respiratory function and prote in degradation in the context of PD. Understanding the relationship between these processes, namely if autophagy can prevent mitochondrial dysfunction and accumulation of aggregated proteins, is essential for design of better therapeutics. Improving autophagic efficiency is interesting approach to treating PD; however, this warrants further studies in both the PD cybrids and in new models being created from sporadic PD patient samples. The failure of therapies derived from models of familial PD indicates that studying sporadic PD in order to understand the pathogenic mechanisms in these patients is essential for the development of therapeutics that will benefit the entire PD patient community. The mechanistic insights gained from the studies described in this dissertation shed important light on the relevant pathways for the study and treatment of sporadic PD. 


\section{References}

Abbott, R. D., et al., 2001. Frequency of bowel movements and the future risk of Parkinson's disease. Neurology. 57, 456-62.

Abeliovich, A., et al., 2000a. Mice lacking alpha-synuclein display functional deficits in the nigrostriatal do pamine system. Neuron. 25, 239-52.

Abeliovich, H., et al., 2000b. Dissection of auto phagosome biogenesis into distinct nucleation and expansion steps. J Cell Biol. 151, 1025-34.

Alafuzoff, I., et al., 2009. Staging/typing of Lewy body related al pha-synuclein pathology: a study of the BrainNet Europe Consortium. Acta neuropathologica. 117, 635-52.

Alvarez-Erviti, L., et al., 2010. Chaperone-mediated auto phagy markers in Parkinson disease brains. Archives of neurology. 67, 1464-72.

Ancolio, K., et al., 2000. Alpha-synuclein and the Parkinson's disease-related mutant Ala53Thr-alpha-synuclein do not undergo proteasomal degradation in HEK293 and neuronal cells. Neuroscience letters. 285, 79-82.

Anderson, S., et al., 1981. Sequence and organization of the human mitochondrial genome. Nature. 290, 457-65.

Anglade, P., et al., 1997. Apoptosis and autophagy in nigral neurons of patients with Parkinson's disease. Histology and histopathology. 12, 25-31.

Armon, T., et al., 1990. Assembly of the $26 \mathrm{~S}$ complex that degrades proteins ligated to ubiquitin is accompanied by the formation of ATPase activity. The Journal of biological chemistry. 265, 20723-6.

Arthur, C. R., et al., 2009. Parkinson's disease brain mitochondria have impaired respiraso me assembly, age-related increases in distribution of oxidative damage to mtDNA and no differences in heteroplasmic mtDNA mutation abundance. Molecular neurodegeneration. 4, 37.

Ashley, N., et al., 2005. Detection of mitochondrial DNA depletion in living human cells using PicoGreen staining. Experimental cell research. 303, 432-46.

Asin-Cayuela, J., Gustafsson, C. M., 2007. Mitochondrial transcription and its regulation in mammalian cells. Trends in biochemical sciences. 32, 111-7.

$\mathrm{Au}$, W. L., Calne, D. B., 2005. A reassessment of the Lewy body. Acta neurologica Taiwanica. 14, 40-7.

Bai, Y., et al., 2001. Lack of complex I activity in human cells carrying a mutation in MtDNA-encoded ND4 subunit is corrected by the Saccharomyces cerevisiae NADH-quinone oxidoreductase (NDI1) gene. The Journal of biological chemistry. 276, 38808-13.

Banerjee, R., et al., 2009. Mitochondrial dysfunction in the limelight of Parkinson's disease pathogenesis. Biochimica et biophysica acta. 1792, 651-63.

Barber-Singh, J., et al., 2010. Protective Role of rAAV-NDI1, Serotype 5, in an Acute MPTP Mouse Parkinson's Model. Parkinson's disease. 2011, 438370. 
Barber-Singh, J., et al., 2009. Neuroprotective effect of long-term NDI 1 gene expression in a chronic mouse model of Parkinson disorder. Rejuvenation research. 12, 259-67.

Barja, G., Herrero, A., 1998. Localization at complex I and mechanism of the higher free radical production of brain nonsynaptic mitochondria in the short-lived rat than in the longevous pigeon. Journal of bioenergetics and biomembranes. $30,235-43$.

Bastin, J., et al., 2008. Activation of peroxisome proliferator-activated receptor pathway stimulates the mitochondrial respiratory chain and can correct deficiencies in patients' cells lacking its components. The Journal of clinical endocrinology and metabolism. 93, 1433-41.

Beal, M. F., 2011. Neuroprotective effects of creatine. Amino acids. 40,1305-13.

Bender, A., et al., 2006. High levels of mitochondrial DNA deletions in substantia nigra neurons in aging and Parkinson disease. Nature genetics. 38, 515-7.

Bennett, M. C., et al., 1999. Degradation of alpha-synuclein by proteasome. The Journal of biological chemistry. 274, 33855-8.

Bereiter-Hahn, J. r., 1997. Distribution and dynamics of mitocho ndrial nucleoids in animal cells in culture. Experimental Biology Online. 1,1-17.

Bertrand, S. J., et al., 2011. Endogenous amyloidogenesis in long-term rat hippocampal cell cultures. BMC neuroscience. 12, 38.

Betarbet, R., et al., 2000. Chronic systemic pesticide exposure reproduces features of Parkinson's disease. Nature neuroscience. 3, 1301-6.

Beyer, K., 2006. Alpha-synuclein structure, posttranslational modification and alternative splicing as aggr egation enhancers. Acta neuropathologica. 112, 237-51.

Bhaskar, P. T., Hay, N., 2007. The two TORCs and Akt. Developmental cell. 12 , 487502.

Biedler, J. L., et al., 1973. Morphology and growth, tumorigenicity, and cytogenetics of human neuroblasto ma cells in continuous culture. Cancer research. 33, 2643-52.

Bjorkoy, G., et al., 2005. p62/SQSTM1 forms protein aggregates degraded by autophagy and has a protective effect on huntingtin-induced cell death. The Journal of cell biology. 171, 603-14.

Blandini, F., Armentero, M. T., 2012. Animal models of Parkinson's disease. The FEBS journal. 279, 1156-66.

Bodner, R. A., et al., 2006. Pharmacological promotion of inclusion formation: a therapeutic approach for Huntington's and Parkinson's diseases. Proceedings of the National Academy of Sciences of the United States of America. 103, 4246-51.

Bogenhagen, D. F., et al., 2008. The layered structure of human mitochondrial D NA nucleoids. The Journal of biological chemistry. 283, 3665-75.

Boland, B., et al., 2008. Autophagy induction and autophago some clearance in neurons: relationship to auto phagic pathology in Alzheimer's disease. The Journal of neuroscience : the official journal of the Society for Neuroscience. 28, 6926-37. 
Bonifati, V., et al., 2003. Mutations in the DJ-1 gene associated with auto somal recessive early-onset parkinso nism. Science. 299, 256-9.

Borland, M. K., et al., 2009. Relation ships among molecular genetic and respiratory properties of Parkinson's disease cybrid cells show similarities to Parkinson's brain tissues. Biochimica et biophysica acta. 1792, 68-74.

Borland, M. K., et al., 2008. Chronic, low-dose rotenone reproduces Lewy neurites found in early stages of Parkinson's disease, reduces mitochondrial movement and slowly kills differentiated SH-SY5Y neural cells. Molecular neurodegeneration. 3, 21.

Braak, E., et al., 2001. alpha-synuclein immunopositive Parkinson's disease-related inclusion bodies in lower brain stem nuclei. Acta neuropathologica. 101, 195201.

Braak, H., et al., 2006. Gastric alpha-synuclein immunor eactive inclusions in Meissner's and Auerbach's plexuses in cases staged for Parkinson's diseaserelated brain pathology. Neuroscience letters. 396, 67-72.

Braak, H., et al., 2003. Staging of brain pathology related to sporadic Parkinson's disease. Neurobiology of aging. 24, 197-211.

Brand, M. D., et al., 1993. Control of the effective $\mathrm{P} / 0$ ratio of oxidative phosphorylation in liver mitochondria and hepatocytes. The Biochemical journal. 291 ( Pt 3), 739-48.

Brand, M. D., Nicholls, D. G., 2011. Assessing mitochondrial dysfunction in cells. The Biochemical journal. 435, 297-312.

Brunk, U. T., Terman, A., 2002. The mitochondrial-lysoso mal axis theory of aging: accumulation of damaged mitochondria as a result of imperfect autophagocytosis. European journal of biochemistry / FEBS. 269, 1996-2002.

Campello, S., Scorrano, L., 2010. Mitochondrial shape changes: orchestrating cell pathophysiology. EMBO reports. 11, 678-84.

Cannon, J. R., Greenamyre, J. T., 2010. Neurotoxic in vivo models of Parkinson's disease recent advances. Progress in brain research. 184, 17-33.

Capaldi, R. A., et al., 2004. Immunological approaches to the characterization and diagnosis of mitochondrial disease. Mitochondrion. 4, 417-26.

Castellani, R., et al., 1996. Glycoxidation and oxidative stress in Parkinson disease and diffuse Lewy body disease. Brain research. 737, 195-200.

Chartier-Harlin, M. C., et al., 2004. Alpha-synuclein locus duplication as a cause of familial Parkinson's disease. Lancet. 364, 1167-9.

Chaturvedi, R. K., Beal, M. F., 2012. Mitochondria targeted therapeutic approaches in Parkinson's and Huntington's diseases. Molecular and cellular neurosciences.

Chau, V., et al., 1989. A multiubiquitin chain is confined to specific lysine in a targeted short-lived protein. Science. 243, 1576-83.

Chaudhuri, K. R., Schapira, A. H., 2009. Non-motor symptoms of Parkinson's disease: dopaminergic pathophysiology and treatment. Lancet neurology. 8, 464-74.

Cherra, S. J., 3rd, Chu, C. T., 2008. Autophagy in neuroprotection and neurodegeneration: A question of balance. Future neurology. 3, 309-323.

Cherra, S. J., 3rd, et al., 2013. Mutant LRRK2 elicits calcium imbalance and depletion of dendritic mitochondria in neurons. The American journal of pathology. $182,474-84$. 
Chu, C. T., 2006. Autophagic stress in neuronal injury and disease. Journal of neuropathology and experimental neurology. 65, 423-32.

Chu, C. T., et al., 2000. Ubiquitin immunochemistry as a diagnostic aid for community pathologists evaluating patients who have dementia. Modern pathology : an official journal of the United States and Canadian Academy of Pathology, Inc. 13, 420-6.

Chu, C. T., et al., 2007. Beclin 1-independent pathway of damage-induced mitophagy and autophagic stress: implications for neuro degeneration and cell death. Autophagy. 3, 663-6.

Chu, Y., et al., 2009. Alterations in lyso somal and protea somal markers in Parkinson's disease: relationship to alpha-synuclein inclusions. Neurobiology of disease. 35, 385-98.

Codogno, P., et al., 2012. Canonical and non-canonical autophagy: variations on a common theme of self-eating? Nature reviews. Molecular cell biology. 13, 712.

Conway, K. A., et al., 2000. Fibrils formed in vitro from al pha-synuclein and two mutant forms linked to Parkinson's disease are typical amyloid. Biochemistry. 39, 2552-63.

Cookson, M. R., 2012. Cellular effects of LRRK2 mutations. Biochemical Society transactions. 40, 1070-3.

Cronin-Furman, E. N., et al., 2013. Mitochondrial quality, dynamics and functional capacity in Parkinson's disease cybrid cell lines selected for Lewy body expression. Molecular neurodegeneration. 8, 6.

Cuervo, A. M., et al., 2004. Impaired degradation of mutant al pha-synuclein by chaperone-mediated autophagy. Science. 305, 1292-5.

Dagda, R. K., et al., 2009. Loss of PINK1 function promotes mitophagy through effects on oxidative stress and mitochondrial fission. J Biol Chem. 284, 13843-55.

Dauer, W., Przedborski, S., 2003. Parkinson's disease: mechanisms and models. Neuron. 39, 889-909.

de la Fuente-Fernandez, R., et al., 1998. The role of the Lewy body in idiopathic Parkinsonism. Parkinsonism \& related disorders. 4, 73-7.

de Vries, S., Grivell, L. A., 1988. Purification and characterization of a rotenoneinsensitive NADH:Q6 oxidoreductase from mitochondria of Saccharomyces cerevisiae. European journal of biochemistry / FEBS. 176, 377-84.

Del Tredici, K., Braak, H., 2012. Spinal cord lesions in sporadic Parkinson's disease. Acta neuropathologica. 124, 643-64.

Del Tredici, K., et al., 2002. Where does parkinson disease pathology begin in the brain? Journal of neuropathology and experimental neurology. 61, 413-26.

Den Hartog Jager, W. A., Bethlem, J., 1960. The distribution of Lewy bodies in the central and autonomic nervous systems in idiopathic paralysis agitans. J Neurol Neurosurg Psychiatry. 23, 283-90.

Dexter, D. T., et al., 1989. Basal lipid peroxidation in substantia nigra is increased in Parkinson's disease. Journal of neurochemistry. 52, 381-9.

Diaz, F., Moraes, C. T., 2008. Mitochondrial biogenesis and turnover. Cell calcium. 44, 24-35. 
Dickson, D. W., 2012. Parkinson's disease and parkinsonism: neuropathology. Cold Spring Harbor perspectives in medicine. 2.

Ding, T. T., et al., 2002. Annular alpha-synuclein protofibrils are produced when spherical protofibrils are incubated in solution or bound to brain-derived membranes. Biochemistry. 41, 10209-17.

Doehner, J., et al., 2012. Extrusion of misfolded and aggregated proteins--a protective strategy of aging neurons? The European journal of neuroscience. 35, 1938-50.

Dranka, B. P., et al., 2011. Assessing bioenergetic function in response to oxidative stress by metabolic profiling. Free radical biology \& medicine. 51, 1621-35.

Dranka, B. P., et al., 2012. Alterations in bioenergetic function induced by Parkinson's disease mimetic compounds: lack of correlation with superoxide generation. Journal of neurochemistry. 122, 941-51.

Duffy, P. E., Tennyson, V. M., 1965. Phase and Electron Microscopic Observations of Lewy Bodies and Melanin Granules in the Substantia Nigra and Locus Caeruleus in Parkinson's Disease. Journal of Neuropathology \& Experimental Neurology. 24, 398-414.

Dunn, W. A., Jr., 1990. Studies on the mechanisms of autophagy: formation of the autophagic vacuole. The Journal of cell biology. 110,1923-33.

Dyall, S. D., et al., 2004. Ancient invasions: from endosymbionts to organelles. Science. 304, 253-7.

Elbaz, A., Tranchant, C., 2007. Epidemiologic studies of environmental exposures in Parkinson's disease. Journal of the neurological sciences. 262, 37-44.

Eskelinen, E. L., 2008. To be or not to be? Examples of incorrect identification of autophagic compartments in conventional transmission electron microscopy of mammalian cells. Autophagy. 4, 257-60.

Esteves, A. R., et al., 2010. Mitochondrial respiration and respiration-associated proteins in cell lines created through Parkinson's subject mitochondrial transfer. Journal of neurochemistry. 113, 674-82.

Exner, N., et al., 2012. Mitochondrial dysfunction in Parkinson's disease: molecular mechanisms and pathophysiological consequences. The EMBO journal. 31, 3038-62.

Fager qvist, T., et al., 2013. Monoclonal antibodies selective for alpha-synuclein oligomers/protofibrils recognize brain pathology in Lewy body disorders and alpha-synuclein transgenic mice with the disease-causing A30P mutation. Journal of neurochemistry. 126, 131-44.

Ferrick, D. A., et al., 2008. Advances in measuring cellular bioenergetics using extracellular flux. Drug discovery today. 13, 268-74.

Fiskum, G., et al., 2003. Mitochondrial mechanisms of neural cell death and neuroprotective interventions in Parkinson's disease. Annals of the New York Academy of Sciences. 991, 111-9.

Floyd, R. A., Carney, J. M., 1992. Free radical damage to protein and DNA: mechanisms involved and relevant observations on brain undergoing oxidative stress. Annals of neurology. 32 Suppl, S22-7.

Forno, L. S., 1996. Neuropathology of Parkinson's disease. Journal of neuropathology and experimental neurology. 55, 259-72. 
Fuentealba, L. C., et al., 2008. Asymmetric mitosis: Unequal segregation of proteins destined for degradation. Proceedings of the National Academy of Sciences of the United States of America. 105, 7732-7.

Galloway, P. G., et al., 1992. Filaments of Lewy bodies contain insoluble cytoskeletal elements. Am J Pathol. 140, 809-22.

Galluzzi, L., et al., 2012. Mitochondrial control of cellular life, stress, and death. Circulation research. 111, 1198-207.

Galvin, J. E., et al., 1999. Pathobiology of the Lewy body. Advances in neurology. 80, 313-24.

Gaspari, M., et al., 2004. The transcription machinery in mammalian mitochondria. Biochimica et biophysica acta. 1659, 148-52.

Gerencser, A. A., et al., 2009. Quantitative microplate-based respirometry with correction for oxygen diffusion. Analytical chemistry. 81, 6868-78.

Giasson, B. I., et al., 2006. Biochemical and pathological char acterization of Lrrk 2. Annals of neurology. 59, 315-22.

Giasson, B. I., et al., 2000. Oxidative damage linked to neurodegeneration by selective alpha-synuclein nitration in synucleinopathy lesions. Science. 290, 985-9.

Gomes, L. C., et al., 2011. During autophagy mitochondria elongate, are spared from degradation and sustain cell viability. Nature cell biology. 13, 589-98.

Gray, M. W., et al., 1999. Mitochondrial evolution. Science. 283, 1476-81.

Greffard, S., et al., 2010. A stable proportion of Lewy body bearing neurons in the substantia nigra suggests a model in which the Lewy body causes neuronal death. Neurobiology of aging. 31, 99-103.

Guarente, L., 2007. Sirtuins in aging and disease. Cold Spring Harbor symposia on quantitative biology. 72, 483-8.

Haas, R. H., et al., 1995. Low platelet mitochondrial complex I and complex II/III activity in early untreated Parkinson's disease. Ann Neurol. 37, 714-22.

Hailey, D. W., et al., 2010. Mitochondria supply membranes for auto phagosome biogenesis during starvation. Cell. 141, 656-67.

Halliday, G., et al., 2012. Evaluation of the Braak hypothesis: how far can it explain the pathogenesis of Parkinson's disease? Expert review of neurotherapeutics. 12, 673-86.

Hansen, C., Li, J. Y., 2012. Beyond alpha-synuclein transfer: pathology propagation in Parkinson's disease. Trends in molecular medicine. 18, 248-55.

Harding, A. J., et al., 2002a. Visual hallucinations in Lewy body disease relate to Lewy bodies in the temporal lobe. Brain : a journal of neurology. 125, 391-403.

Harding, A. J., et al., 2002b. Clinical correlates of selective pathology in the amygdala of patients with Parkinson's disease. Brain : a journal of neurology. 125, 2431-45.

Harrower, T., Barker, R. A., 2005. Cell therapi es for neurological disease--from bench to clinic to bench. Expert opinion on biological therapy. 5, 289-91.

Harrower, T. P., et al., 2005. Lewy bodies in Parkinson's disease: protectors or perpetrators? Experimental neurology. 195, 1-6.

Hartmann, A., 2004. Postmortem studies in Parkinson's disease. Dialogues in clinical neuroscience. 6, 281-93. 
He, J., et al., 2012. Mitochondrial nucleoid interacting proteins support mitochondrial protein synthesis. Nucleic acids research. 40, 6109-21.

Higashi, S., et al., 2011. Localization of MAP1-LC3 in vulnerable neurons and Lewy bodies in brains of patients with dementia with Lewy bodies. Journal of neuropathology and experimental neurology. 70, 264-80.

Hindle, J. V., 2010. Ageing, neurodegeneration and Parkinson's disease. Age and ageing. 39, 156-61.

Hirsch, E., et al., 1988. Melanized dopaminergic neurons are differentially susceptible to degeneration in Parkinson's disease. Nature. 334, 345-8.

Hock, M. B., Kralli, A., 2009. Transcriptional control of mitochondrial biogenesis and function. Annual review of physiology. 71, 177-203.

Huang, Y., et al., 2012. Macroauto phagy in sporadic and the genetic form of Parkinson's disease with the A53T alpha-synuclein mutation. Translational neurodegeneration. 1, 2.

Hughes, A. J., et al., 1992. Accuracy of clinical diagno sis of idiopathic Parkinson's disease: a clinico-pathological study of 100 cases. J Neurol Neurosurg Psychiatry. 55, 181-4.

Hundt, E., et al., 1980. Bio synthesis of cytochrome $\mathrm{c}$ oxidase in isolated rat hepatocytes. FEBS letters. 115, 95-9.

Ischiropoulos, H., 2003. Oxidative modifications of alpha-synuclein. Annals of the New York Academy of Sciences. 991, 93-100.

Jalava, A., et al., 1992. Staurosporine induces a neuronal phenotype in SH-SY5Y human neuroblasto ma cells that resembles that induced by the phorbol ester 12-0-tetradecanoyl phorbol-13 acetate (TPA). FEBS letters. 300, 114-8.

Javitch, J. A., et al., 1985. Parkinsonism-inducing neurotoxin, N-methyl-4-phenyl1,2,3,6 -tetrahydropyridine: uptake of the metabolite N-methyl-4phenylpyridine by dopamine neurons explains selective toxicity. Proceedings of the National Academy of Sciences of the United States of America. 82, 2173-7.

Jellinger, K. A., 2009. Formation and development of Lewy pathology: a critical update. Journal of neurology. $256 \mathrm{Suppl} \mathrm{3,270-9.}$

Jung, C. H., et al., 2009. ULK-Atg13-FIP200 complexes mediate mTOR signaling to the autophagy machinery. Mol Biol Cell. 20, 1992-2003.

Junn, E., et al., 2002. Parkin accumulation in aggresomes due to proteasome impairment. The Journal of biological chemistry. 277, 47870-7.

Kabeya, Y., et al., 2000. LC3, a mammalian ho mologue of yeast Apg8p, is localized in autophagosome membranes after processing. The EMBO journal. 19, 5720-8.

Kaushik, S., Cuervo, A. M., 2012. Chaperone-mediated autophagy: a unique way to enter the lysosome world. Trends in cell biology. 22, 407-17.

Keen ey, P. M., et al., 2009. Cybrid models of Parkinson's disease show variable mitochondrial biogenesis and genotype-respiration relationships. Experimental neurology. 220, 374-82.

Keeney, P. M., et al., 2006. Parkinson's disease brain mitochond rial complex I has oxidatively damaged subunits and is functionally impaired and misassembled. The Journal of neuroscience : the official journal of the Society for Neuroscience. 26, 5256-64. 
Khurana, R., et al., 2001. Is Congo red an amyloid-specific dye? The Journal of biological chemistry. 276, 22715-21.

Kim, P. K., et al., 2008. Ubiquitin signals autophagic degradation of cytosolic proteins and peroxisomes. Proceedings of the National Academy of Sciences of the United States of America. 105, 20567-74.

Kish, S. J., et al., 1985. Glutathio ne peroxidase activity in Parkinson's disease brain. Neuroscience letters. 58, 343-6.

Kitada, T., et al., 1998. Mutations in the parkin gene cause autosomal recessive juvenile parkinsonism. Nature. 392, 605-8.

Klein, C., Schlossmacher, M. G., 2007. Parkinson disease, 10 years after its genetic revolution: multiple clues to a complex disorder. Neurology. 69, 2093-104.

Klucken, J., et al., 2012. Alpha-synuclein aggregation involves a bafilomycin A 1sensitive autophagy pathway. Autophagy. 8, 754-66.

Knott, A. B., et al., 2008. Mitochondrial fragmentation in neurodegeneration. Nature reviews. Neuroscience. 9, 505-18.

Korolchuk, V. I., et al., 2011. Lysoso mal positioning coordinates cellular nutrient responses. Nature cell biology. 13, 453-60.

Kramer, M. L., Schulz-Schaeffer, W. J., 2007. Presynaptic al pha-synuclein aggregates, not Lewy bodies, cause neurodegeneration in dementia with Lewy bodies. The Journal of neuroscience : the official journal of the Society for Neuroscience. 27, 1405-10.

Kraytsberg, Y., et al., 2006. Mitochondrial DNA deletions are abundant and cause functional impairment in aged human substantia nigra neurons. Nature genetics. 38, 518-20.

Kuma, A., et al., 2007. LC3, an autophagoso me marker, can be incorporated into protein aggregates independent of auto phagy: caution in the interpretation of LC3 localization. Autophagy. 3, 323-8.

Kurz, T., et al., 2007. Autophagy, ageing and apoptosis: the role of oxidative stress and lyso somal iron. Archives of biochemistry and biophysics. 462, 220-30.

Lagouge, M., Larsson, N. G., 2013. The role of mitochondrial DNA mutations and free radicals in disease and ageing. Journal of internal medicine. 273, 529-43.

Lan, D. M., et al., 2012. Effect of trehalo se on PC1 2 cells overexpressing wild-type or A53T mutant al pha-synuclein. Neurochemical research. 37, 2025-32.

Langston, J. W., et al., 1983. Chronic Parkinsonism in humans due to a product of meperidine-analog synthesis. Science. 219, 979-80.

Langston, J. W., et al., 1999. Evidence of active nerve cell degeneration in the substantia nigra of humans years after 1-methyl-4-phenyl-1,2,3,6tetrahydropyridine exposure. Annals of neurology. 46, 598-605.

Larsson, N. G., 2010. So matic mitochondrial DNA mutations in mammalian aging. Annual review of biochemistry. 79, 683-706.

Lee, H. J., et al., 2013. Autophagic failure promotes the exocytosis and intercellular transfer of alpha-synuclein. Experimental \& molecular medicine. 45, e22.

Lees, A. J., et al., 2009. Parkinso n's disease. Lancet. 373, 2055-66.

Lemasters, J. J., 2005. Selective mitochondrial autophagy, or mito phagy, as a targeted defense against oxidative stress, mitochondrial dysfunction, and aging. Rejuvenation Res. 8, 3-5. 
Leroy, E., et al., 1998. The ubiquitin pathway in Parkinson's disease. Nature. 395, 451-2.

Lesage, S., Brice, A., 2009. Parkinson's disease: from monogenic forms to genetic susceptibility factors. Human molecular genetics. 18, R48-59.

Lin, C. J., et al., 2012. Inhibition of mitochondria- and endoplasmic reticulum stressmediated auto phagy augments temozolo mide-induced apoptosis in glioma cells. PloS one. 7, e38706.

Linnane, A. W., et al., 1989. Mitochondrial DNA mutations as an important contributor to ageing and degenerative diseases. Lancet. 1, 642-5.

Liu, S., Wong-Riley, M., 1994. Nuclear-encoded mitochondrial precursor protein: intramitochondrial delivery to dendrites and axon terminals of neurons and regulation by neuronal activity. The Journal of neuroscience : the official journal of the Society for Neuroscience. 14, 5338-51.

Lu, L., et al., 2005. Gene expression profiling of Lewy body-bearing neurons in Parkinson's disease. Experimental neurology. 195, 27-39.

Lum, J. J., et al., 2005. Growth factor regulation of autophagy and cell survival in the absence of apoptosis. Cell. 120,237-48.

Marella, M., et al., 2007. Mechanism of cell death caused by complex I defects in a rat dopaminergic cell line. The Journal of biological chemistry. 282, 24146-56.

Marella, M., et al., 2008. Protection by the NDI1 gene against neurodegeneration in a rotenone rat mo del of Parkinson's disease. PloS one. 3, e1433.

Marella, M., et al., 2010. Successful amelio ration of mitochondrial optic neuropathy using the yeast NDI1 gene in a rat animal model. PloS one. 5, e11472.

Marres, C. A., et al., 1991. Isolation and inactivation of the nuclear gene encoding the rotenone-insensitive inter nal NADH: ubiquino ne oxidoreductase of mitochondria from Saccharomyces cerevisiae. European journal of biochemistry / FEBS. 195, 857-62.

Martinez-Vicente, M., et al., 2005. Protein degradation and aging. Experimental gerontology. 40, 622-33.

McNaught, K. S., Jenner, P., 2001. Proteasomal function is impaired in substantia nigra in Parkinson's disease. Neuroscience letters. 297, 191-4.

McNaught, K. S., et al., 2002. Aggresome-related biogenesis of Lewy bodies. The European journal of neuroscience. 16, 2136-48.

Melendez, A., Levine, B., 2009. Autophagy in C. el egans. WormBook : the online review of C. el egans biology. 1-26.

Miller, S. W., et al., 1996. Creation and characterization of mitochondrial DNAdepleted cell lines with "neuronal-like" properties. Journal of neurochemistry. 67,1897-907.

Ming, G. L., et al., 2011. Cellular reprogramming: recent advances in modeling neurological diseases. The Journal of neuroscience : the official journal of the Society for Neuro science. 31, 16070-5.

Mitchell, P., 1961. Coupling of phosphorylation to el ectron and hydrogen transfer by a chemi-osmotic type of mechanism. Nature. 191, 144-8.

Mizuno, Y., et al., 2008. Progress in the pathogenesis and genetics of Parkinson's disease. Philosophical transactions of the Royal Society of London. Series B, Biological sciences. 363, 2215-27. 
Mizuno, Y., et al., 1987. Effects of 1-methyl-4-phenyl-1,2,3,6-tetrahydropyridine and 1-methyl-4-phenylpyridinium ion on activities of the enzymes in the electron transport system in mouse brain. Journal of neurochemistry. 48, 1787-93.

Moran, M., et al., 2012. Mitochondrial respiratory chain dysfunction: implications in neurodegeneration. Free radical biology \& medicine. 53, 595-609.

Mouli, P. K., et al., 2009. Frequency and selectivity of mitochondrial fusion are key to its quality maintenance function. Biophys J. 96, 3509-18.

Mudo, G., et al., 2012. Transgenic expression and activation of PGC-1alpha protect dopaminergic neurons in the MPTP mouse model of Parkinson's disease. Cellular and molecular life sciences : CMLS. 69, 1153-65.

Muller, T., 2010. New small molecules for the treatment of Parkinson's disease. Expert opinion on investigational drugs. 19,1077-86.

Narendra, D., et al., 2008. Parkin is recruited selectively to impaired mitochondria and promotes their autophagy. J Cell Biol. 183, 795-803.

Noda, T., Ohsumi, Y., 1998. Tor, a phosphatidylinositol kinase homologue, controls autophagy in yeast. The Journal of biological chemistry. 273,3963-6.

Olanow, C. W., et al., 2004. Lewy-body formation is an aggreso me-related process: a hypothesis. Lancet neurology. 3, 496-503.

Olanow, C. W., et al., 2009. The scientific and clinical basis for the treatment of Parkinson disease (2009). Neurology. 72, S1-136.

Pahlman, S., et al., 1984. Retinoic acid-induced differentiation of cultured human neuroblasto ma cells: a comparison with phorbolester-induced differentiation. Cell differentiation. 14, 135-44.

Paisan-Ruiz, C., et al., 2004. Cloning of the gene containing mutations that cause PARK8-linked Parkinson's disease. Neuron. 44, 595-600.

Palacino, J. J., et al., 2004. Mitochondrial dysfunction and oxidative damage in parkin-deficient mice. J Biol Chem. 279, 18614-22.

Pankiv, S., et al., 2007. p62/SQSTM1 binds directly to Atg8/LC3 to facilitate degradation of ubiquitinated protein aggregates by auto phagy. The Journal of biological chemistry. 282, 24131-45.

Papa, S., De Rasmo, D., 2013. Complex I deficiencies in neurological disorders. Trends in molecular medicine. 19, 61-9.

Papa, S., et al., 2012. The oxidative phosphorylation system in mammalian mitochondria. Advances in experimental medicine and biology. 942, 3-37.

Park, J., et al., 2006. Mitochondrial dysfunction in D rosophila PINK1 mutants is complemented by parkin. Nature. 441,1157-61.

Park, J. S., et al., 2007. Yeast NDI1 improves oxidative phosphorylation capacity and increases protection against oxidative stress and cell death in cells carrying a Leber's hereditary optic neuropathy mutation. Biochimica et biophysica acta. $1772,533-42$.

Parker, W. D., Jr., et al., 1989. Abnormalities of the el ectron transport chain in idiopathic Parkinson's disease. Ann Neurol. 26, 719-23.

Petrovitch, H., et al., 2009. Bowel movement frequency in late-life and substantia nigra neuron density at death. Movement disor ders : official journal of the Movement Disorder Society. 24, 371-6. 
Picard, M., et al., 2013. Mitochondrial morphology transitions and functions: implications for retrogra de signaling? American journal of physiology. Regulatory, integrative and comparative physiology. 304, R393-406.

Plomp, P. J., et al., 1989. Energy dependence of different steps in the autophagic lysosomal pathway. The Journal of biological chemistry. 264, 6699-704.

Plomp, P. J., et al., 1987. Energy dependence of autophagic protein degradation in isolated rat hepatocytes. European journal of biochemistry / FEBS. 164, 197 203.

Pollanen, M. S., et al., 1993. Pathology and biology of the Lewy body. Journal of neuropathology and experimental neurology. 52, 183-91.

Polymeropoulos, M. H., et al., 1997. Mutation in the alpha-synuclein gene identified in families with Parkinson's disease. Science. 276, 2045-7.

Poole, A. C., et al., 2008. The PINK1/Parkin pathway regulates mitochondrial morphology. Proceedings of the National Academy of Sciences of the United States of America. 105, 1638-43.

Postuma, R. B., et al., 2013. Prodromal autono mic symptoms and signs in Parkinson's disease and dementia with Lewy bodies. Movement disorders : official journal of the Movement Disorder Society.

Ravikumar, B., et al., 2010. Plasma membrane contributes to the for mation of preautophagoso mal structures. Nature cell biology. 12, 747-57.

Richardson, J. R., et al., 2007. Obligato ry role for com plex I inhibition in the dopaminergic neurotoxicity of 1-methyl-4-phenyl-1,2,3,6-tetrahydropyridine (MPTP). Toxicological sciences : an official journal of the Society of Toxicology. 95, 196-204.

Ross, G. W., et al., 2008. Association of olfactory dysfunction with risk for future Parkinson's disease. Annals of neurology. 63, 167-73.

Rudenko, I. N., et al., 2012. The G2385R variant of leucine-rich repeat kinase 2 associated with Parkinson's disease is a partial loss-of-function mutation. The Biochemical journal. 446, 99-111.

Rujano, M. A., et al., 2006. Polarised asymmetric inheritance of accumulated protein damage in higher eukaryotes. PLoS biology. 4 , e417.

Sanchez, I., et al., 2003. Pivotal role of oligo merization in expanded polyglutamine neurodegenerative disorders. Nature. 421, 373-9.

Sanz, A., et al., 2010. Expression of the yeast NADH dehydrogenase Ndi1 in Drosophila confers increased lifespan independently of dietary restriction. Proceedings of the National Academy of Sciences of the United States of America. 107, 9105-10.

Sarkar, S., et al., 2007. Small molecules enhance autophagy and reduce toxicity in Huntington's disease models. Nature chemical biology. 3, 331-8.

Sarkar, S., et al., 2009. Auto phagic clearance of aggregate-prone proteins associated with neurodegeneration. Methods in enzymology. 453, 83-110.

Satoh, M., Kuroiwa, T., 1991. Organization of multiple nucleoids and DNA molecules in mitochondria of a human cell. Experimental cell research. 196, 137-40.

Savitt, J. M., et al., 2006. Diagnosis and treatment of Parkinson disease: molecules to medicine. The Journal of clinical investigation. 116, 1744-54. 
Scarpulla, R. C., 2008. Nuclear control of respiratory chain expression by nuclear respiratory factors and PGC-1-related coactivator. Annals of the New York Academy of Sciences. 1147, 321-34.

Schapira, A. H., et al., 1990a. Mitochondrial complex I deficiency in Parkinson's disease. J Neurochem. 54, 823-7.

Schapira, A. H., et al., 1990b. Anatomic and disease specificity of NADH CoQ1 reductase (complex I) deficiency in Parkinson's disease. J Neurochem. 55, 2142-5.

Schneider, L., et al., 2011. Differentiation of SH-SY5Y cells to a neuronal phenotype changes cellular bioenergetics and the response to oxidative stress. Free radical biology \& medicine. 51, 2007-17.

Seo, B. B., et al., 1998. Molecular remedy of complex I defects: rotenone-insensitive internal NADH-quinone oxidoreductase of Saccharo myces cerevisiae mitochondria restores the NADH oxidase activity of complex I-deficient mammalian cells. Proceedings of the National Academy of Sciences of the United States of America. 95, 9167-71.

Seo, B. B., et al., 2006. The single subunit NADH dehydrogenase reduces generation of reactive oxygen species from complex I. FEBS letters. 580, 6105-8.

Seo, B. B., et al., 1999. Modulation of oxidative pho sphorylation of human kidney 293 cells by transfection with the internal rotenone-insensitive NADHquinone oxi doreductase (NDI1) gene of Saccharo myces cerevisiae. Biochimica et biophysica acta. 1412, 56-65.

Seo, B. B., et al., 2004. Functional expression of the single subunit NADH dehydrogenase in mitochondria in vivo: a potential therapy for complex I deficiencies. Human gene therapy. 15, 887-95.

Seo, B. B., et al., 2002. A single-subunit NADH-quinone oxidoreductase renders resistance to mammalian nerve cells against complex I inhibition. Molecular therapy : the journal of the American Society of Gene Therapy. 6, 336-41.

Seo, B. B., et al., 2000. U se of the NADH-quinone oxidoreductase (NDI1) gene of Saccharomyces cerevisiae as a possible cure for complex I defects in human cells. The Journal of biological chemistry. 275, 37774-8.

Shaikh, S., Nicholson, L. F., 2008. Advanced glycation end products induce in vitro cross-linking of alpha-synuclein and accelerate the process of intracellular inclusion body formation. Journal of neuroscience research. 86, 2071-82.

Sherer, T. B., et al., 2007. Mechanism of toxicity of pesticides acting at complex I: relevance to environmental etiologies of Parkinson's disease. Journal of neurochemistry. 100, 1469-79.

Shin, J. H., et al., 2011. PARIS (ZNF 746) repression of PGC-1alpha contributes to neurodegeneration in Parkinson's disease. Cell. 144, 689-702.

Shults, C. W., 2006. Lewy bodies. Proceedings of the National Academy of Sciences of the United States of America. 103, 1661-8.

Singleton, A. B., et al., 2003. al pha-Synuclein locus triplication causes Parkinson's disease. Science. 302, 841.

Smith, R. A., Murphy, M. P., 2010. Animal and human studies with the mitochondriatargeted antioxidant MitoQ. Annals of the New York Academy of Sciences. 1201, 96-103. 
Soldner, F., et al., 2009. Parkinson's disease patient-derived induced pluripotent stem cells free of viral reprogramming factors. Cell. 136, 964-77.

Spillantini, M. G., et al., 1997. Alpha-synuclein in Lewy bodies. Nature. 388, 839-40.

Srivastava, S., et al., 2009. PGC-1alpha/beta induced expression partially compensates for respiratory chain defects in cells from patients with mitochondrial disor ders. Human molecular genetics. 18, 1805-12.

Svenningsson, P., et al., 2012. Cognitive impairment in patients with Parkinson's disease: diagno sis, biomarkers, and treatment. Lancet neurology. 11, 697707.

Swerdlow, R. H., et al., 2013. Glycolysis-respiration relationships in a neuroblastoma cell line. Biochimica et biophysica acta. 1830, 2891-8.

Swerdlow, R. H., et al., 1996. Origin and functional consequences of the complex I defect in Parkinson's disease. Annals of neurology. 40, 663-71.

Takahashi, K., et al., 2007. Induction of pluripotent stem cells from adult human fibroblasts by defined factors. Cell. 131, 861-72.

Tanik, S. A., et al., 2013. Lewy body-like alpha-synuclein aggregates resist degradation and impair macroautophagy. The Journal of biological chemistry.

Tanji, K., et al., 2011. Alteration of autophago somal proteins (LC3, GABARAP and GATE-16) in Lewy body disease. Neurobiology of disease. 43, 690-7.

Tanner, C. M., et al., 2011. Rotenone, paraquat, and Parkinson's disease. Environmental health perspectives. 119, 866-72.

Terman, A., Brunk, U. T., 2006. Oxidative stress, accumulation of biological 'garbage', and aging. Antioxidants \& redox signaling. 8, 197-204.

Terman, A., et al., 2003. Mitocho ndrial recycling and aging of cardiac myocytes: the role of auto phagocytosis. Experimental gerontology. 38, 863-76.

Tofaris, G. K., Spillantini, M. G., 2007. Physiological and pathological properties of alpha-synuclein. Cellular and molecular life sciences : CMLS. 64, 2194-201.

Tompkins, M. M., Hill, W. D., 1997. Contribution of somal Lewy bodies to neuronal death. Brain research. 775, 24-9.

Tooze, S. A., Yoshimori, T., 2010. The origin of the auto phagosomal membrane. Nature cell biology. 12, 831-5.

Trimmer, P. A., Bennett, J. P., Jr., 2009. The cybrid model of sporadic Parkinson's disease. Experimental neurology. 218, 320-5.

Trimmer, P. A., et al., 2004. Parkinson's disease transgenic mitochondrial cybrids generate Lewy inclusion bo dies. Journal of neurochemistry. 88, 800-12.

Trimmer, P. A., et al., 2000. Abnormal mitochondrial morphology in sporadic Parkinson's and Alzheimer's disease cybrid cell lines. Experimental neurology. 162, 37-50.

Tsang, A. H., Chung, K. K., 2009. Oxidative and nitrosative stress in Parkinson's disease. Biochimica et biophysica acta. 1792, 643-50.

Twig, G., et al., 2008a. Fission and selective fusion govern mitochondrial segregation and elimination by autophagy. The EMBO journal. 27, 433-46.

Twig, G., et al., 2008b. Mitochondrial fusion, fission and autophagy as a quality control axis: the bioenergetic view. Biochimica et biophysica acta. 1777, 1092-7. 
Valente, E. M., et al., 2004a. Hereditary early-onset Parkinson's disease caused by mutations in PINK1. Science. 304, 1158-60.

Valente, E. M., et al., 2004b. PINK1 mutations are associated with sporadic earlyonset parkinso nism. Ann Neurol. 56, 336-41.

van Sluijters, D. A., et al., 2000. Amino-acid-dependent signal transduction. The Biochemical journal. 351 Pt 3, 545-50.

Wakabayashi, K., et al., 2007. The Lewy body in Parkinson's disease: molecules implicated in the formation and degradation of alpha-synuclein aggregates. Neuropathology. 27, 494-506.

Waldmeier, P., et al., 2006. Recent clinical failures in Parkinson's disease with apoptosis inhibitors underline the need for a paradigm shift in drug discovery for neurodegenerative diseases. Biochemical pharmacology. 72, 1197-206.

Wang, L., et al., 2013. LC3 fluorescent puncta in autophagosomes or in protein aggregates can be distinguished by FRAP analysis in living cells. Autophagy. 9.

Warburg, 0., 1956. On the origin of cancer cells. Science. 123, 309-14.

Wareski, P., et al., 2009. PGC-1\{alpha\} and PGC-1 beta\} regulate mitochondrial density in neurons. The Journal of biological chemistry. 284, 21379-85.

Watanabe, Y., et al., 2012. p62/SQSTM1-dependent auto phagy of Lewy body-like alpha-synuclein inclusions. PloS one. 7, e52868.

Weisiger, R. A., Fridovich, I., 1973. Superoxide dismutase. Organelle specificity. The Journal of biological chemistry. 248, 3582-92.

Wenz, T., 2011. Mitochondria and PGC-1 alpha in Aging and Age-Associated Diseases. Journal of aging research. 2011,810619.

Wenz, T., et al., 2008. Activation of the PPAR/PGC-1 alpha pathway prevents a bioenergetic deficit and effectively improves a mitochondrial myopathy phenotype. Cell metabolism. 8, 249-56.

Winklhofer, K. F., Haass, C., 2010. Mitochondrial dysfunction in Parkinson's disease. Biochimica et biophysica acta. 1802, 29-44.

Winner, B., et al., 2011. In vivo demonstration that al pha-synuclein oligo mers are toxic. Proceedings of the National Aca demy of Sciences of the United States of America. 108, 4194-9.

Winslow, A. R., et al., 2010. alpha-Synuclein impairs macroautophagy: implications for Parkinson's disease. The Journal of cell biology. 190, 1023-37.

Wong, E. S., et al., 2008. Autophagy-mediated clearance of aggresomes is not a universal phenomenon. Human molecular genetics. 17, 2570-82.

$\mathrm{Wu}$, Z., et al., 1999. Mechanisms controlling mitochondrial biogenesis and respiration through the thermogenic coactivator PGC-1. Cell. 98, 115-24.

Xilouri, M., Stefanis, L., 2011. Auto phagic pathways in Parkinson disease and related disorders. Expert reviews in molecular medicine. 13, e8.

Xiong, N., et al., 2011. Potential autophagy enhancers attenuate rotenone-induced toxicity in SH-SY5Y. Neuroscience. 199, 292-302.

Xiong, N., et al., 2013. The role of autophagy in Parkinson's disease: rotenone-based modeling. Behavioral and brain functions : BBF. 9, 13. 
Yadava, N., Nicholls, D. G., 2007. Spare respiratory capacity rather than oxidative stress regulates glutamate excitotoxicity after partial respiratory inhibition of mitochondrial complex I with rotenone. The Journal of neuroscience: the official journal of the Society for Neuro science. 27, 7310-7.

Yagi, T., Matsuno-Yagi, A., 2003. The proton-translocating NADH-quino ne oxidoreductase in the respiratory chain: the secret unlocked. Biochemistry. 42, 2266-74.

Yang, Z., Klionsky, D. J., 2009. An overview of the molecular mechanism of autophagy. Current to pics in microbiology and immunology. 335, 1-32.

Yoritaka, A., et al., 1996. Immuno histochemical detection of 4-hydroxynonenal protein adducts in Parkinson disease. Proceedings of the National Academy of Sciences of the United States of America. 93, 2696-701.

Youle, R. J., van der Bliek, A. M., 2012. Mitochondrial fis sion, fusion, and stress. Science. 337, 1062-5.

$\mathrm{Yu}, \mathrm{W}$. H., et al., 2009. Metabolic activity determines efficacy of macroautophagic clearance of pathological oligomeric alpha-synuclein. The American journal of pathology. $175,736-47$.

Zatloukal, K., et al., 2002. p62 Is a common component of cytoplasmic inclusions in protein aggregation diseases. The American journal of pathology. 160, 25563.

Zhang, Y., et al., 2000. Parkin functions as an E2-dependent ubiquitin- protein ligase and promotes the degradation of the synaptic vesicle-associated protein, CDCrel-1. Proceedings of the National Academy of Sciences of the United States of America. 97, 13354-9.

Zhdanov, A. V., et al., 2011. Bafilomycin A1 activates respiration of neuro nal cells via uncoupling associated with flickering depolarization of mitochondria. Cellular and molecular life sciences : CMLS. 68, 903-17.

Zheng, B., et al., 2010. PGC-1alpha, a potential therapeutic target for early intervention in Parkinson's disease. Science translational medicine. 2, 52 ra73.

Zhou, J., et al., 2011. Changes in the solubility and pho sphorylation of alphasynuclein over the course of Parkinson's disease. Acta neuropathologica. 121, 695-704.

Zick, M., et al., 2009. Cristae formation-linking ultrastructure and function of mitochondria. Biochimica et biophysica acta. 1793, 5-19.

Zimprich, A., et al., 2004. Mutations in LRRK2 cause auto somal-dominant parkinsonism with pleomorphic pathology. Neuron. 44, 601-7.

Zurita Rendon, O., Shoubridge, E. A., 2012. Early complex I assembly defects result in rapid turnover of the ND1 subunit. Human molecular genetics. 21, 3815-24. 